\title{
The Global Prevalence of Depression, Suicide Ideation, and Attempts in the Military Forces: A Systematic Review and Meta-Analysis of Cross Sectional Studies
}

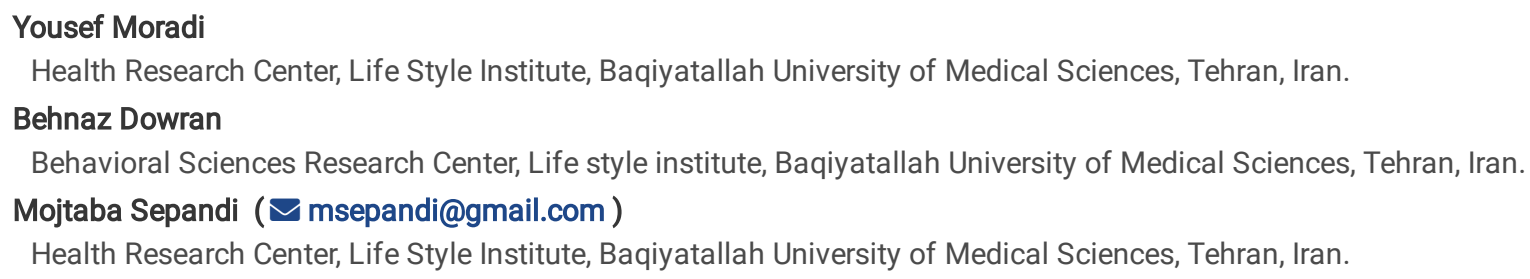

\section{Research Article}

Keywords: Suicide ideation, Suicide Attempts, Depression, Military, Systematic Review and Meta-Analysis

Posted Date: March 12th, 2021

DOI: https://doi.org/10.21203/rs.3.rs-274705/v1

License: (c) (1) This work is licensed under a Creative Commons Attribution 4.0 International License. Read Full License 


\section{Abstract \\ Background}

Given the wide range of depressive disorders, suicidal ideation and suicide attempts in various military studies around the world, determining the exact prevalence of these disorders in line with health planning as well as care and treatment service designing for military forces can be useful. The aim of the present meta-analysis was to determine the pooled prevalence of depressive disorders, suicide thoughts, and attempts in the military.

\section{Methods}

The present systematic review and meta-analysis study was performed based on PRISMA criteria in 5 steps of the search strategy, screening and selection of articles, data extraction, evaluation of article quality and meta-analysis. International databases (PubMed, Scopus, Web of Sciences, Embase, Cochrane, Psyclnfo) were searched using related keywords extracted from Mesh and Emtree. After screening and final selection of articles, data were extracted and qualitative evaluation was performed using the NOS checklist.

\section{Results}

The results of meta-analysis showed that the prevalence of depression in active military forces and veterans was $23 \%$ (\%95 Cl: $20-26 \%$ and $20 \%$ (\%95 Cl: $18-22 \%)$, respectively. In addition, the prevalence of suicidal ideation and attempts in the military was $11 \%$ (\%95 Cl: $10-13 \%)$ and $11 \%$ (\%95 Cl: 9-13\%), respectively. The prevalence of suicide ideation and attempts in drug-using military was 18\% (\%95 Cl: 7-33 \%) and 30\% (\%95 Cl: 23-36\%), respectively. The prevalence of suicidal ideation and attempts in military consuming alcohol were $9 \%$ (\%95 Cl: 4-13\%) and $8 \%$ (\%95 Cl: 7-10\%), respectively. In militaries with AIDS / HIV, the prevalence of suicide attempts was 5\% (\%95 Cl: 4-8 \%).

\section{Conclusion}

Therefore, it is necessary to develop and design training and intervention programs in order to increase the awareness of the military, especially veterans, to prevent the occurrence of suicide and depression.

\section{Background}

Mental health is one of the basic pillars of health that requires a useful, effective and satisfactory individual life (1). Promoting the mental health of a society requires the dynamism and growth of that society (2). Paying attention to mental health in all areas of life, including personal, social and professional ones, is important and debatable. One of the areas in which mental health is concerned is the job and profession. Based on the available findings, mental disorders are one of the most important and significant causes of diseases and it was predicted that in 2020 the share of mental and neurological disorders in the total burden of diseases would increase by $50 \%(3-5)$. Therefore, attention to mental health is important in all areas of the individual, social and professional life $(6,7)$. One of the important stressful environmental stimuli that can cause chronic stress and significantly affect people's psyche is the type of the job in which a person is engaged so that if the stress caused by the work environment becomes excessive, it can cause physical and psychological effects on the individual and his/her family. It can be said that it endangers the health of the individual and threatens the organizational goals and leads to a decrease in the quality of the individual's performance. Research has shown that several factors affect job stress (8-10). These include shift work, or jobs which are full of environmental stress. If a person is not able to cope with the stressors of his/her job, he/she will suffer from multiple physical, psychological and behavioral consequences. In this regard, the military forces of different countries perform different missions according to the conditions of the region and their countries, but during this decade, in order to provide higher defense capability and presence at greater depths and distances away from the origin, military forces need to design and make tools with higher ranges and quality, which need their own engineering and ergonomic requirements (11-13). One of the most important issues in this field, which can be the first question and has caused intellectual and executive concern of military officials and commanders, is to identify and implement methods to increase the durability and maintain the performance of military personnel so that during increasing mission time, their efficiency will not be disrupted or effectively reduced $(14,15)$. This is where the role of military psychology and psychological variables affecting the effectiveness of military forces become clearer $(16,17)$. Psychological assessment and mental disorders are very important among military personnel because war, living in operational conditions, multiple combat missions, being away from the family, captivity, wounding and environmental restrictions, as well as cultural differences are always parts of the military life. Therefore, due to this type of lifestyle, burnout, job stress and various mental disorders such as depression and suicide are very common among them $(18,19)$. For this reason, conducting epidemiological and psychological research among military personnel is of great importance. In addition, accurately determining the prevalence of mental disorders in this group can help health policy makers and health professionals to take more effective and appropriate control and treatment measures $(20,21)$. On the other hand, the military forces' awareness of the occurrence of these disorders can be effective in performing appropriate health behaviors, suitable lifestyle changes, and ultimately in preventing further occurrence of these disorders. So far, various descriptive and

Page 2/50 
analytical studies have been conducted in the world with the aim of determining the prevalence of mental disorders, especially depression and suicide in servicemen in various fields such as naval, land and air forces, but the results of these studies were very contradictory. In addition, the range of prevalence, considering the results of these studies, was very wide such that the exact prevalence of mental disorders in this group could not be considered (22-24). Therefore, it was very important to conduct a systematic review and meta-analysis in order to more accurately determine the prevalence of depressive and suicidal disorders (suicide thoughts and attempts). On the other hand, by considering the results of the systematic review and meta-analysis study, it was possible to create appropriate knowledge and determine the exact prevalence of these disorders in the military in order to allocate health care resources and screening services to the military. The purpose of this systematic review and metaanalysis was to determine the exact prevalence of depressive disorders and suicide in the military of the world.

\section{Methods}

This systematic review and meta-analysis was based on the standards Preferred Reporting Items for Systematic Reviews and Meta-Analyzes (PRISMA) and Meta-analyzes of Observational Studies in Epidemiology (MOOSE) (25-27).

\section{Search syntax and Search strategy}

This study was a systematic review and meta-analysis that aimed to accurately determine the prevalence of depression, suicide thoughts, and suicide attempts in the military. Finding of articles published from January 1990 to December 2020 was done in 5 electronic databases (PubMed (Medline), Scopus, Web of science, Embase, Ovid, Cochrane, Psyclnfo) using the main keywords of Depression (synonymous with "Depressively", "Depressive Disorder", "Depressed", "Depressive Symptoms", "Emotional Depression", "Unipolar Depression", "Neurotic Depression", "Depressive Syndromes", "Endogenous Depression", and "Depressive Neurosis)", suicide thoughts and attempts (with synonyms of "Suicide", "Suicidality", "Attempted Suicide", "Para Suicide", "Completed Suicide", and "Thoughts of Suicide"), as well as Military people (with synonyms of "Armed Forces Personnel", "Military Personnel", "Air Forces Personnel", "Veterans", "Submariners", "Marines", "Navy Personnel", "Sailors", "Soldiers", "Military Deployment", and "Coast Guard ").

\section{Eligibility criteria's}

Cross-sectional studies aim of which were to determine and report the prevalence or frequency of depressive disorders, suicide thoughts, or attempts in the military were entered into the research. This means that studies in which the study population was the military, and the main outcome of which was to determine the prevalence of depression and suicide (suicide thoughts or attempts) were considered in this review. Studies included in the systematic review should have reported desired outcomes (the prevalence of depressive disorders and suicide) in frequency and percentage.

Studies that had reported depressive and suicidal disorders (suicidal ideation or attempts) as a mean and standard deviation, or ones of which target population was other than military personnel themselves (such as military spouses or their children), retrospective, or prospective studies with the cohort base, cohort studies, case studies, clinical trials, systematic reviews, letters to the editor, editorial, and survey studies over 5 years were excluded from the present research.

The search strategy was independently developed and conducted by two of the authors and the existing differences were resolved in the opinion of the third researcher with more experience. Gray Literature-related sites and databases such as Psyclnfo, Google Scholar, World Health Organization (WHO) were also searched. In addition, Manual Search was performed at the end of the screening process.

\section{Screening and selection of articles}

First, an Endnote library (Version 8) was created to collect articles, remove duplicates, and review titles and abstracts. In the first screening step, the review of titles and abstracts was independently done by one of the researchers and $10 \%$ of the reviewed articles were randomly reviewed by the second researcher and the differences were resolved by discussing and referring to the third person if necessary. The screened references were selected for full-text review if they contained the desired information in their title or abstract. In the next step, the full text was separately reviewed by two of the authors. Data were extracted from the eligible studies and entered into Excel 2016.

\section{Data extraction}

In order to extract the data, first a checklist was prepared with the opinion of experts in relation to the data extracted from the articles and then the data were extracted. Required information included author's name, year of publication of articles, statistical population of study, country of study, type of study, instrument for measuring depression and suicide disorders in the military, sample size, average age of military personnel and quality evaluation score of primary studies.

\section{Risk of Bias}


Two of the authors conducted a qualitative evaluation of the studies based on the Newcastle - Ottawa Quality Assessment Scale (NOS)

checklist $(28,29)$. This checklist has designed to evaluate the quality of observational studies, especially cross-sectional ones. This tool examined each study with 6 items in three groups, including: how to select study samples, how to compare and analyze study groups, and how to measure and analyze the desired outcome. Each of these items was given a score of 1 if it was observed in the studies, and the maximum score for each study was 9 points. In case of discrepancies in the score assigned to the published articles and for reaching an agreement, the discussion method and the third researcher were used.

\section{Meta-analysis}

In this research, for meta-analysis, first, the prevalence values were extracted from the selected studies, and then the standard error prevalence values for each study were calculated using the Metan command. In addition to performing this order, the number of patients with the desired outcome (depression or suicide) was extracted from the total sample size in each of the studies to perform the Metaprop order. In this research, the model of DerSimonian-Liard random effects was used to estimate the pooled prevalence of depression and suicide (estimate of $95 \%$ confidence interval) in military personnel using the Metaprop and Metan commands in Stata 16. Cochrane Q and I2 tests were used to investigate the heterogeneity and variance between the studies selected for meta-analysis. Funnel Plot and Egger test were used to evaluate the diffusion bias. Also, meta-regression analysis and diagram were used to investigate the relationship between age variables with the estimated pooled prevalence.

\section{Results}

\section{Qualitative Results}

In this systematic review and meta-analysis study after conducting the search strategy, screening of articles was done based on title, abstract and full text. On the topic of depression in the military, 3422, 1773, 343, 445, and 11 articles were retrieved at PubMed, Embase, Web of Sciences, Scopus, and Psyclnfo, respectively. On the subject of suicide (thoughts or attempts), 1551, 1036, 170, 346, and 10 articles were retrieved on PubMed, Embase, Web of Sciences, Scopus, and PsycInfo, respectively. After removing duplicates, in the Endnote software, screening based on the title of the articles was started with 5275 studies related to depression and 3022 studies related to suicide. After screening articles by the title, 3531 studies related to depression and 1842 studies related to suicide were excluded because they were not relevant to the study objectives. 1744 studies related to depression and 1180 studies related to suicide were entered into the screening phase based on the abstract. Finally, at this stage, 2458 articles (a total of 1499 and 959 studies) related to depression and suicide in the military were deleted. After full-text screening, 133 studies related to depression and 58 studies related to suicide in the military remained and were included in the present study for meta-analysis (Fig. 1).

Of the total studies entered into this systematic review on depression, 94 studies in the USA, 4 studies in the UK, 11 studies in Canada, 2 studies in Korea, 2 studies in Turkey, 2 studies in Croatia, 1 study in Russia, 2 studies in China, 1 study in Argentina, 1 study in New Zealand, 1 study in Iran, 1 study in Iraq, 1 study in Norway, 1 study in Australia, 1 study in Greece, 1 study in Sweden, 1 study in Pakistan, 2 studies in Lebanon, 2 studies in Thailand, and 1 study in Finland had been conducted. Also, 84 out of 133 studies related to the prevalence of depression in the military had collected study samples by the available sampling method and the rest of the studies had used the random sampling method. Finally, the whole of these studies had examined 2166819 military personnel of whom 356140 were depressed (Tables 1 and 2). 
Table 1

The study characteristics of included studies about depression

\begin{tabular}{|c|c|c|c|c|c|c|c|c|}
\hline $\begin{array}{l}\text { Authors } \\
\text { (Years) }\end{array}$ & Country & $\begin{array}{l}\text { Type of } \\
\text { Sampling } \\
\text { (Type of } \\
\text { Study) }\end{array}$ & Study Population & $\begin{array}{l}\text { Depression } \\
\text { Assessment } \\
\text { Method }\end{array}$ & $\begin{array}{l}\text { Age } \\
\text { (Mean) }\end{array}$ & Sample size & $\begin{array}{l}\text { Prevalence } \\
\text { of } \\
\text { Depression } \\
(\%)\end{array}$ & $\begin{array}{l}\text { NOS } \\
\text { Score }\end{array}$ \\
\hline $\begin{array}{l}\text { Tredgold, R. } \\
\text { F. }(1941)(65)\end{array}$ & UK & $\begin{array}{l}\text { Convenience } \\
\text { Sampling } \\
\text { (CS) }\end{array}$ & Army men & $\begin{array}{l}\text { Clinical Symptoms } \\
\text { (Interviews) }\end{array}$ & - & 274 & 70 (25.54\%) & 6 \\
\hline $\begin{array}{l}\text { Helzer, J. E. } \\
\text { et al (1976) } \\
(66)\end{array}$ & USA & $\begin{array}{l}\text { Random } \\
\text { Sampling } \\
\text { (CS) }\end{array}$ & Army men & $\begin{array}{l}\text { Clinical Symptoms } \\
\text { (Interviews) }\end{array}$ & - & 470 & $122(26 \%)$ & 7 \\
\hline $\begin{array}{l}\text { Levine, M. E. } \\
(1982)(67)\end{array}$ & USA & $\begin{array}{l}\text { Convenience } \\
\text { Sampling } \\
\text { (CS) }\end{array}$ & Army men & $\begin{array}{l}\text { Beck Depression } \\
\text { Inventory (BDI) }\end{array}$ & 17 & 200 & $36(18 \%)$ & 6 \\
\hline $\begin{array}{l}\text { Deeken, M. G. } \\
\text { et al (1987) } \\
(68)\end{array}$ & USA & $\begin{array}{l}\text { Convenience } \\
\text { Sampling } \\
\text { (CS) }\end{array}$ & Army men & $\begin{array}{l}\text { Zung Self-Rating } \\
\text { Depression Scale }\end{array}$ & - & 298 & $47(15.77 \%)$ & 7 \\
\hline $\begin{array}{l}\text { Ritchie, E. C. } \\
\text { et al (1992) } \\
(69)\end{array}$ & USA & $\begin{array}{l}\text { Random } \\
\text { Sampling } \\
\text { (CS) }\end{array}$ & $\begin{array}{l}\text { Army men with } \\
\text { HIV }\end{array}$ & $\begin{array}{l}\text { Clinical Symptoms } \\
\text { (Interviews) } \\
\text { DSM-III-R }\end{array}$ & - & 50 & $21(42 \%)$ & 7 \\
\hline $\begin{array}{l}\text { Brown, G. R. } \\
\text { et al (1993) } \\
(70)\end{array}$ & USA & $\begin{array}{l}\text { Random } \\
\text { Sampling } \\
\text { (CS) }\end{array}$ & $\begin{array}{l}\text { Air Forces men } \\
\text { with HIV }\end{array}$ & $\begin{array}{l}\text { Structured Interview } \\
\text { Guide for the } \\
\text { Hamilton } \\
\text { Anxiety and } \\
\text { Depression Scales } \\
\text { (SIGH-AD) }\end{array}$ & 35 & 442 & $99(22.4 \%)$ & 8 \\
\hline $\begin{array}{l}\text { McCarroll, J. } \\
\text { E. et al } \\
(1993)(71)\end{array}$ & USA & $\begin{array}{l}\text { Convenience } \\
\text { Sampling } \\
\text { (CS) }\end{array}$ & $\begin{array}{l}\text { Army men and } \\
\text { women }\end{array}$ & $\begin{array}{l}\text { Clinical Symptoms } \\
\text { (Interviews) }\end{array}$ & 25.4 & $\begin{array}{l}1835 \\
\text { Male(1565) } \\
\text { Female(270) }\end{array}$ & $\begin{array}{l}87(4.7 \%) \\
59(3.8 \%) \\
52(19.3 \%)\end{array}$ & 8 \\
\hline $\begin{array}{l}\text { Perconte, S. } \\
\text { T. et al } \\
(1993)(72)\end{array}$ & Russia & $\begin{array}{l}\text { Convenience } \\
\text { Sampling } \\
\text { (CS) }\end{array}$ & $\begin{array}{l}\text { Army men and } \\
\text { women }\end{array}$ & $\begin{array}{l}\text { Beck Depression } \\
\text { Inventory (BDI) }\end{array}$ & 29.25 & 591 & $\begin{array}{l}146(24.70 \\
\%)\end{array}$ & 7 \\
\hline $\begin{array}{l}\text { Serfaty, E. et } \\
\text { al (1995) } \\
(73)\end{array}$ & Argentina & $\begin{array}{l}\text { Random } \\
\text { Sampling } \\
\text { (CS) }\end{array}$ & $\begin{array}{l}\text { Army men and } \\
\text { women }\end{array}$ & NR & NR & 553 & $25(4.5 \%)$ & 7 \\
\hline $\begin{array}{l}\text { Lish, J. D. et } \\
\text { al (1996) } \\
(74)\end{array}$ & USA & $\begin{array}{l}\text { Random } \\
\text { Sampling } \\
\text { (CS) }\end{array}$ & $\begin{array}{l}\text { Army men and } \\
\text { women }\end{array}$ & $\begin{array}{l}\text { Brief self-report } \\
\text { questionnaire } \\
\text { (SCRENNER) }\end{array}$ & 21.2 & 669 & 38 (5.81\%) & 7 \\
\hline $\begin{array}{l}\text { Long, N. et al } \\
(1996)(75)\end{array}$ & $\begin{array}{l}\text { New } \\
\text { Zealand }\end{array}$ & $\begin{array}{l}\text { Random } \\
\text { Sampling } \\
\text { (CS) }\end{array}$ & Army men & $\begin{array}{l}\text { Beck Depression } \\
\text { Inventory (BDI) }\end{array}$ & 50 & 751 & $11(1.46 \%)$ & 7 \\
\hline $\begin{array}{l}\text { Schwartz, D. } \\
\text { A. et al } \\
(1997)(76)\end{array}$ & USA & $\begin{array}{l}\text { Random } \\
\text { Sampling } \\
\text { (CS) }\end{array}$ & $\begin{array}{l}\text { Non-Persian Gulf } \\
\text { War (PGW) } \\
\text { military personnel }\end{array}$ & Self-report & - & 923 & $157(17 \%)$ & 6 \\
\hline $\begin{array}{l}\text { Schwartz, D. } \\
\text { A. et al } \\
(1997)(76)\end{array}$ & USA & $\begin{array}{l}\text { Random } \\
\text { Sampling } \\
\text { (CS) }\end{array}$ & $\begin{array}{l}\text { Persian Gulf War } \\
\text { (PGW) military } \\
\text { personnel }\end{array}$ & Self-report & - & 923 & $99(10.9 \%)$ & 6 \\
\hline
\end{tabular}




\begin{tabular}{|c|c|c|c|c|c|c|c|c|}
\hline $\begin{array}{l}\text { Authors } \\
\text { (Years) }\end{array}$ & Country & $\begin{array}{l}\text { Type of } \\
\text { Sampling } \\
\text { (Type of } \\
\text { Study) }\end{array}$ & Study Population & $\begin{array}{l}\text { Depression } \\
\text { Assessment } \\
\text { Method }\end{array}$ & $\begin{array}{l}\text { Age } \\
\text { (Mean) }\end{array}$ & Sample size & $\begin{array}{l}\text { Prevalence } \\
\text { of } \\
\text { Depression } \\
(\%)\end{array}$ & $\begin{array}{l}\text { NOS } \\
\text { Score }\end{array}$ \\
\hline $\begin{array}{l}\text { David, D. et al } \\
\text { (1999) (77) }\end{array}$ & Croatia & $\begin{array}{l}\text { Convenience } \\
\text { Sampling } \\
\text { (CS) }\end{array}$ & $\begin{array}{l}\text { Veterans after } \\
\text { participation in } \\
\text { Homeland War in } \\
\text { Croatia }\end{array}$ & $\begin{array}{l}\text { The Structured } \\
\text { Clinical Interview } \\
\text { Diagnostic and } \\
\text { Statistical } \\
\text { Manual (SCID) }\end{array}$ & 36.2 & 91 & 35 (38.5 \%) & 7 \\
\hline $\begin{array}{l}\text { Hankin, C. S. } \\
\text { et al (1999) } \\
\text { (78) }\end{array}$ & USA & $\begin{array}{l}\text { Random } \\
\text { Sampling } \\
\text { (CS) }\end{array}$ & Men Veterans & $\begin{array}{l}\text { Center for } \\
\text { Epidemiologic Studies } \\
\text { Depression Scale } \\
\text { (CES-D Scale) }\end{array}$ & 62 & 2160 & $676(31.3 \%)$ & 7 \\
\hline \multirow[t]{2}{*}{$\begin{array}{l}\text { Hourani, L. L. } \\
\text { et al (1999) } \\
(79)\end{array}$} & \multirow[t]{2}{*}{ USA } & \multirow{2}{*}{$\begin{array}{l}\text { Random } \\
\text { Sampling } \\
\text { (CS) }\end{array}$} & \multirow[t]{2}{*}{$\begin{array}{l}\text { Men and Women } \\
\text { in the Navy and } \\
\text { Marine Corps }\end{array}$} & \multirow{2}{*}{$\begin{array}{l}\text { Center for } \\
\text { Epidemiologic Studies } \\
\text { Depression Scale } \\
\text { (CES-D Scale) }\end{array}$} & \multirow[t]{2}{*}{$20-64$} & 782 & $\begin{array}{l}125 \\
(16.08 \%)\end{array}$ & \multirow[t]{2}{*}{7} \\
\hline & & & & & & $\begin{array}{l}\text { Male (321) } \\
\text { Female } \\
(452)\end{array}$ & $\begin{array}{l}29(9 \%) \\
99(22 \%)\end{array}$ & \\
\hline $\begin{array}{l}\text { Curran, G. M. } \\
\text { et al (2000) } \\
(80)\end{array}$ & USA & $\begin{array}{l}\text { Random } \\
\text { Sampling } \\
\text { (CS) }\end{array}$ & Men Veterans & $\begin{array}{l}\text { (Beck Depression } \\
\text { Inventory )BDI( }\end{array}$ & 43 & 298 & $116(39 \%)$ & 7 \\
\hline $\begin{array}{l}\text { Menon, A. S. } \\
\text { et al (2000) } \\
(81)\end{array}$ & USA & $\begin{array}{l}\text { Convenience } \\
\text { Sampling } \\
\text { (CS) }\end{array}$ & Men Veterans & $\begin{array}{l}\text { The Structured } \\
\text { Clinical Interview for } \\
\text { DSM-III-R (SCID-III-R) }\end{array}$ & 55 & 295 & $59(22.8 \%)$ & 6 \\
\hline $\begin{array}{l}\text { Kozaric- } \\
\text { Kovacic, D. et } \\
\text { al (2001) } \\
(82)\end{array}$ & Croatia & $\begin{array}{l}\text { Random } \\
\text { Sampling } \\
\text { (CS) }\end{array}$ & Men Veterans & $\begin{array}{l}\text { The Hamilton } \\
\text { Depression Rating } \\
\text { Scale (HAMD) }\end{array}$ & 34 & 249 & 77 (31 \%) & 7 \\
\hline $\begin{array}{l}\text { Sayar, K. et al } \\
(2001)(83)\end{array}$ & Turkey & $\begin{array}{l}\text { Random } \\
\text { Sampling } \\
\text { (CS) }\end{array}$ & Men Soldiers & $\begin{array}{l}\text { (Beck Depression } \\
\text { Inventory )BDI( }\end{array}$ & 22.7 & 40 & $13(32.5 \%)$ & 7 \\
\hline $\begin{array}{l}\text { Hunter, C. } \\
\text { L.et al (2002) } \\
\text { (84) }\end{array}$ & USA & $\begin{array}{l}\text { Random } \\
\text { Sampling } \\
\text { (CS) }\end{array}$ & Active Duty & $\begin{array}{l}\text { The Patient Health } \\
\text { Questionnaire } \\
\text { (PHQ) } \\
\text { (the self-report version } \\
\text { of the PRIME-MD) }\end{array}$ & 54.15 & 337 & $19(5.6 \%)$ & 7 \\
\hline $\begin{array}{l}\text { Karel, M. J. et } \\
\text { al (2002)(85) }\end{array}$ & USA & $\begin{array}{l}\text { Random } \\
\text { Sampling }\end{array}$ & Men Veterans & $\begin{array}{l}\text { The Geriatric } \\
\text { Depression Scale } \\
\text { (GDS)- } 15 \text { item }\end{array}$ & 69.7 & 967 & $236(24.4 \%)$ & 7 \\
\hline & & Study) & & $\begin{array}{l}\text { Hamilton Depression } \\
\text { Rating Scale } \\
\text { (HDRS)-24 item }\end{array}$ & 69.7 & 967 & $94(9.7 \%)$ & \\
\hline $\begin{array}{l}\text { Kilbourne, A. } \\
\text { M. et al } \\
\text { (2002)(86) }\end{array}$ & USA & $\begin{array}{l}\text { Random } \\
\text { Sampling } \\
\text { (CS) }\end{array}$ & $\begin{array}{l}\text { Veterans with HIV } \\
\text { infection }\end{array}$ & $\begin{array}{l}\text { The } 10 \text {-item Centers } \\
\text { for Epidemiologic } \\
\text { Studies Depression } \\
\text { Scale (CES-D) }\end{array}$ & 49 & 881 & $405(46 \%)$ & 7 \\
\hline $\begin{array}{l}\text { Lehman, C. L. } \\
\text { et al (2002) } \\
\text { (87) }\end{array}$ & USA & $\begin{array}{l}\text { Convenience } \\
\text { Sampling } \\
\text { (CS) }\end{array}$ & $\begin{array}{l}\text { Veterans with } \\
\text { Hepatitis C }\end{array}$ & $\begin{array}{l}\text { The Beck Depression } \\
\text { Inventory } \\
\text { (BDI) }\end{array}$ & 49 & 120 & $53(44.2 \%)$ & 6 \\
\hline
\end{tabular}




\begin{tabular}{|c|c|c|c|c|c|c|c|c|}
\hline $\begin{array}{l}\text { Authors } \\
\text { (Years) }\end{array}$ & Country & $\begin{array}{l}\text { Type of } \\
\text { Sampling } \\
\text { (Type of } \\
\text { Study) }\end{array}$ & Study Population & $\begin{array}{l}\text { Depression } \\
\text { Assessment } \\
\text { Method }\end{array}$ & $\begin{array}{l}\text { Age } \\
\text { (Mean) }\end{array}$ & Sample size & $\begin{array}{l}\text { Prevalence } \\
\text { of } \\
\text { Depression } \\
(\%)\end{array}$ & $\begin{array}{l}\text { NOS } \\
\text { Score }\end{array}$ \\
\hline \multirow{2}{*}{$\begin{array}{l}\text { Muir, A. J. et } \\
\text { al (2002)(88) }\end{array}$} & \multirow[t]{2}{*}{ USA } & \multirow{2}{*}{$\begin{array}{l}\text { Convenience } \\
\text { Sampling } \\
\text { (CS) }\end{array}$} & Veterans with & The Center for & \multirow[t]{2}{*}{47.3} & \multirow[t]{2}{*}{100} & \multirow[t]{2}{*}{$12(12 \%)$} & \multirow[t]{2}{*}{6} \\
\hline & & & Hepatitis C & $\begin{array}{l}\text { Epidemiological } \\
\text { Studies Depression } \\
\text { (CES-D) scale }\end{array}$ & & & & \\
\hline \multirow{2}{*}{$\begin{array}{l}\text { Nguyen, H. A. } \\
\text { et al (2002) } \\
(89)\end{array}$} & \multirow[t]{2}{*}{ USA } & \multirow{2}{*}{$\begin{array}{l}\text { Convenience } \\
\text { Sampling } \\
\text { (CS) }\end{array}$} & Veterans with & \multirow{2}{*}{$\begin{array}{l}\text { Clinical Symptoms } \\
\text { (Interviews) }\end{array}$} & \multirow[t]{2}{*}{46.5} & \multirow[t]{2}{*}{118} & \multirow[t]{2}{*}{$73(62 \%)$} & \multirow[t]{2}{*}{6} \\
\hline & & & Hepatitis C & & & & & \\
\hline \multirow{2}{*}{$\begin{array}{l}\text { Black, D. W. } \\
\text { et al (2004) } \\
(90)\end{array}$} & \multirow[t]{2}{*}{ USA } & $\begin{array}{l}\text { Convenience } \\
\text { Sampling }\end{array}$ & Veterans & $\begin{array}{l}\text { Clinical Symptoms } \\
\text { (Interviews) }\end{array}$ & \multirow[t]{2}{*}{39.3} & \multirow[t]{2}{*}{602} & \multirow[t]{2}{*}{$192(32 \%)$} & \multirow[t]{2}{*}{6} \\
\hline & & (CS) & & DSM-III-R & & & & \\
\hline \multirow{2}{*}{$\begin{array}{l}\text { Gerson, S. et } \\
\text { al (2004) } \\
\text { (91) }\end{array}$} & \multirow[t]{2}{*}{ USA } & \multirow{2}{*}{$\begin{array}{l}\text { Convenience } \\
\text { Sampling } \\
\text { (CS) }\end{array}$} & $\begin{array}{l}\text { Elderly veterans } \\
\text { (Male) }\end{array}$ & $\begin{array}{l}\text { Mental Health } \\
\text { Inventory (MHI) }\end{array}$ & \multirow[t]{2}{*}{69.6} & \multirow[t]{2}{*}{839} & \multirow[t]{2}{*}{$273(32.5 \%)$} & \multirow[t]{2}{*}{8} \\
\hline & & & & & & & & \\
\hline \multirow{2}{*}{$\begin{array}{l}\text { Rowan, P. J. } \\
\text { et al (2004) } \\
(92)\end{array}$} & USA & $\begin{array}{l}\text { Convenience } \\
\text { Sampling }\end{array}$ & Veterans with & $\begin{array}{l}\text { The Zung Self-report } \\
\text { Depression Scale }\end{array}$ & 51 & 580 & $93(16 \%)$ & 7 \\
\hline & & & Hepatitis C & $(\mathrm{SDS})$ & & & & \\
\hline $\begin{array}{l}\text { Smith, T. C. et } \\
\text { al }(2004)(93)\end{array}$ & USA & $\begin{array}{l}\text { Random } \\
\text { Sampling }\end{array}$ & US & & 55 & 8893 & $\begin{array}{l}1642(18.5 \\
\%)\end{array}$ & 8 \\
\hline & & (CS) & Military & $\begin{array}{l}\text { Patient Health } \\
\text { Questionnaire }\end{array}$ & & & & \\
\hline & & & & (PHQ) & & & & \\
\hline $\begin{array}{l}\text { Vafaee, B.et } \\
\text { al (2004)(94) }\end{array}$ & Iran & $\begin{array}{l}\text { Convenience } \\
\text { Sampling } \\
\text { (CS) }\end{array}$ & $\begin{array}{l}\text { Disabled veterans } \\
\text { male }\end{array}$ & $\begin{array}{l}\text { The Zung Self-report } \\
\text { Depression Scale } \\
\text { (SDS) }\end{array}$ & 38 & 100 & $71(71 \%)$ & 5 \\
\hline $\begin{array}{l}\text { Forman- } \\
\text { Hoffman, V. } \\
\text { L. et al }\end{array}$ & USA & $\begin{array}{l}\text { Convenience } \\
\text { Sampling }\end{array}$ & Veterans & $\begin{array}{l}\text { Structured Clinical } \\
\text { Interview for DSM } \\
\text { Disorders (SCID-IV) }\end{array}$ & 39.1 & 602 & $85(14.11 \%)$ & 6 \\
\hline (2005) (95) & & (CS) & & & & & & \\
\hline Goulet, J. L. & USA & Convenience & Veterans with HIV & - & 47.1 & 20627 & $5776(28 \%)$ & 7 \\
\hline (96) & & & Veterans with & - & 46.9 & 4489 & $1975(44 \%)$ & \\
\hline & & & Hepatitis C & & & & & \\
\hline $\begin{array}{l}\text { Rowan, P. J. } \\
\text { et al (2005) }\end{array}$ & USA & $\begin{array}{l}\text { Convenience } \\
\text { Sampling }\end{array}$ & Veterans with & $\begin{array}{l}\text { The Beck Depression } \\
\text { Inventory }\end{array}$ & 52 & 62 & $6(10 \%)$ & 5 \\
\hline & & (CS) & Hepatitis C & (BDI) & & & & \\
\hline $\begin{array}{l}\text { Williams, R. } \\
\text { M. et al }\end{array}$ & USA & $\begin{array}{l}\text { Convenience } \\
\text { Sampling }\end{array}$ & Veterans with & $\begin{array}{l}\text { The Beck Depression } \\
\text { Inventory }\end{array}$ & 55.1 & 451 & $100(22.2 \%)$ & 7 \\
\hline (2005)(98) & & & Multiple sclerosis & & & & & \\
\hline $\begin{array}{l}\text { Xiong, H. et } \\
\text { al }(2005)(99)\end{array}$ & China & $\begin{array}{l}\text { Random } \\
\text { Sampling }\end{array}$ & $\begin{array}{l}\text { Young adult males } \\
\text { during their } 8 \text { week }\end{array}$ & $\begin{array}{l}\text { The Zung Self-report } \\
\text { Depression Scale }\end{array}$ & 20 & 1107 & $279(25.2 \%)$ & 6 \\
\hline & & & $\begin{array}{l}\text { field military } \\
\text { training }\end{array}$ & & & & & \\
\hline $\begin{array}{l}\text { Grieger, T. A. } \\
\text { et al (2006) } \\
(100)\end{array}$ & USA & $\begin{array}{l}\text { Convenience } \\
\text { Sampling }\end{array}$ & $\begin{array}{l}\text { U.S. soldiers were } \\
\text { injured in combat }\end{array}$ & $\begin{array}{l}\text { The nine-item Patient } \\
\text { Health Questionnaire } \\
\text { depression scale }\end{array}$ & 26.94 & 301 & $28(9.3 \%)$ & 5 \\
\hline & & (CS) & & & & & & \\
\hline $\begin{array}{l}\text { Hoge, C. W. et } \\
\text { al (2006) }\end{array}$ & USA & $\begin{array}{l}\text { Random } \\
\text { Sampling }\end{array}$ & $\begin{array}{l}\text { Army soldiers and } \\
\text { Marines }\end{array}$ & The PRIME-MD & 31.2 & 303905 & $15930(5.24$ & 8 \\
\hline (101) & & (CS) & & $\begin{array}{l}\text { Patient Health } \\
\text { Questionnaire }\end{array}$ & & & & \\
\hline & & & & (PHQ) & & & & \\
\hline
\end{tabular}




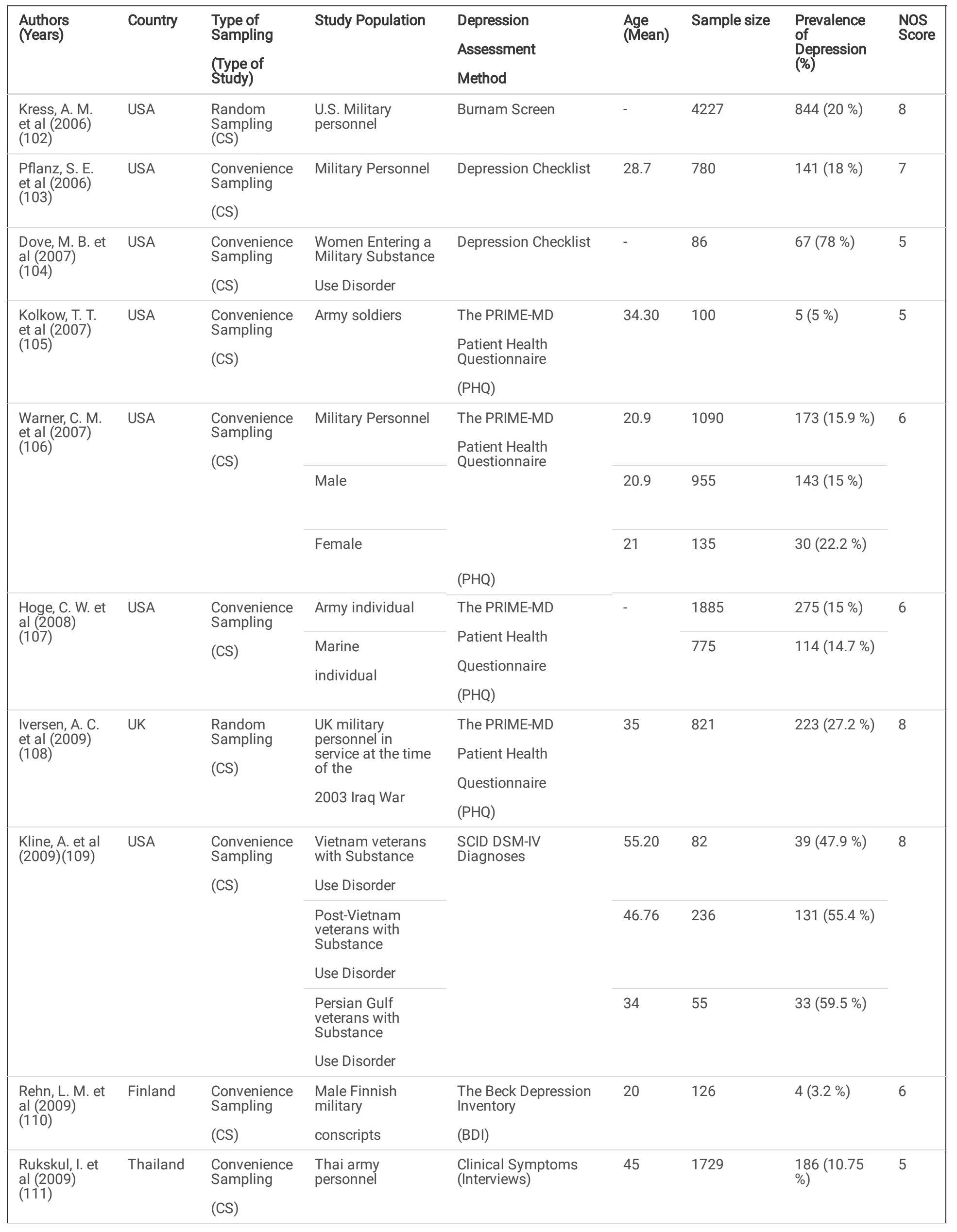




\begin{tabular}{|c|c|c|c|c|c|c|c|c|}
\hline $\begin{array}{l}\text { Authors } \\
\text { (Years) }\end{array}$ & Country & $\begin{array}{l}\text { Type of } \\
\text { Sampling } \\
\text { (Type of } \\
\text { Study) }\end{array}$ & Study Population & $\begin{array}{l}\text { Depression } \\
\text { Assessment } \\
\text { Method }\end{array}$ & $\begin{array}{l}\text { Age } \\
\text { (Mean) }\end{array}$ & Sample size & $\begin{array}{l}\text { Prevalence } \\
\text { of } \\
\text { Depression } \\
(\%)\end{array}$ & $\begin{array}{l}\text { NOS } \\
\text { Score }\end{array}$ \\
\hline $\begin{array}{l}\text { Rukskul, I. } \\
(2010)(112)\end{array}$ & Thailand & $\begin{array}{l}\text { Convenience } \\
\text { Sampling } \\
\text { (CS) }\end{array}$ & $\begin{array}{l}\text { Thai army } \\
\text { personnel }\end{array}$ & $\begin{array}{l}\text { Clinical Symptoms } \\
\text { (Interviews) }\end{array}$ & 45 & 213 & 7 (3.3 \%) & 5 \\
\hline $\begin{array}{l}\text { Fikretoglu, D. } \\
\text { et al (2010) } \\
(113)\end{array}$ & Canada & $\begin{array}{l}\text { Convenience } \\
\text { Sampling } \\
\text { (CS) }\end{array}$ & $\begin{array}{l}\text { Canadian } \\
\text { Community Health } \\
\text { Survey-Canadian } \\
\text { Forces } \\
\text { Supplement } \\
\text { (CCHS-CF) }\end{array}$ & $\begin{array}{l}\text { Diagnostic and } \\
\text { Statistical Manual of } \\
\text { Mental Disorders-IV } \\
\text { (DSM-IV) }\end{array}$ & - & 8441 & $\begin{array}{l}1257(14.9 \\
\%)\end{array}$ & 8 \\
\hline $\begin{array}{l}\text { Haskell, S. G. } \\
\text { et al (2010) } \\
(114)\end{array}$ & USA & $\begin{array}{l}\text { Convenience } \\
\text { Sampling } \\
\text { (CS) }\end{array}$ & $\begin{array}{l}\text { War Veterans of } \\
\text { Iraq and } \\
\text { Afghanistan }\end{array}$ & $\begin{array}{l}\text { Clinical Symptoms } \\
\text { (Interviews) }\end{array}$ & $\begin{array}{l}32 \\
32 \\
30\end{array}$ & $\begin{array}{l}\text { Total (1229) } \\
\text { Male (1032) } \\
\text { Female } \\
(197)\end{array}$ & $\begin{array}{l}472(38.4 \%) \\
380(36.8 \%) \\
92(46.7 \%)\end{array}$ & 7 \\
\hline $\begin{array}{l}\text { Luxton, D. D. } \\
\text { et al (2010) } \\
(115)\end{array}$ & USA & $\begin{array}{l}\text { Convenience } \\
\text { Sampling } \\
\text { (CS) }\end{array}$ & $\begin{array}{l}\text { Active duty } \\
\text { Soldiers } \\
\text { between } \\
\text { March } 2006 \text { and } \\
\text { July } 2009 .\end{array}$ & $\begin{array}{l}\text { The PRIME-MD } \\
\text { Patient Health } \\
\text { Questionnaire } \\
\text { (PHQ) }\end{array}$ & 27.37 & $\begin{array}{l}\text { Total (6943) } \\
\text { Male (6427) } \\
\text { Female } \\
(516)\end{array}$ & $\begin{array}{l}704(10.1 \%) \\
646(10.0 \%) \\
58(46.7 \%)\end{array}$ & 7 \\
\hline $\begin{array}{l}\text { Maguen, S. et } \\
\text { al (2010) } \\
(116)\end{array}$ & USA & $\begin{array}{l}\text { Convenience } \\
\text { Sampling } \\
\text { (CS) }\end{array}$ & $\begin{array}{l}\text { Iraq } \\
\text { and Afghanistan } \\
\text { Veterans Enrolled } \\
\text { in Veterans Affairs }\end{array}$ & $\begin{array}{l}\text { Diagnostic and } \\
\text { Statistical Manual of } \\
\text { Mental Disorders-IV } \\
\text { (DSM-IV) }\end{array}$ & 31.47 & $\begin{array}{l}\text { Total } \\
(329049) \\
\begin{array}{l}\text { Male } \\
(288348)\end{array}\end{array}$ & $\begin{array}{l}57051 \\
(17.33 \%) \\
\begin{array}{l}47876(17 \\
\%)\end{array}\end{array}$ & 8 \\
\hline & & & & & 29.41 & $\begin{array}{l}\text { Female } \\
\text { (40701) }\end{array}$ & $9175(23 \%)$ & \\
\hline $\begin{array}{l}\text { Stecker, T. et } \\
\text { al (2010) } \\
(117)\end{array}$ & Lebanon & $\begin{array}{l}\text { Convenience } \\
\text { Sampling } \\
\text { (CS) }\end{array}$ & $\begin{array}{l}\text { Iraq/Afghanistan } \\
\text { veterans } \\
\text { Iraq/Afghanistan } \\
\text { Veterans with } \\
\text { Alcohol } \\
\text { Use Disorder }\end{array}$ & $\begin{array}{l}\text { Diagnostic and } \\
\text { Statistical Manual of } \\
\text { Mental Disorders-IV } \\
\text { (DSM-IV) }\end{array}$ & 34.4 & $\begin{array}{l}293861 \\
118332\end{array}$ & $\begin{array}{l}36900(12.5 \\
\%) \\
4568(3.8 \%)\end{array}$ & 6 \\
\hline $\begin{array}{l}\text { Burnett- } \\
\text { Zeigler, I. et al } \\
(2011)(118)\end{array}$ & USA & $\begin{array}{l}\text { Random } \\
\text { Sampling } \\
\text { (CS) }\end{array}$ & $\begin{array}{l}\text { Afghanistan and } \\
\text { Iraq Veterans } \\
\text { Iraq/Afghanistan } \\
\text { Veterans with } \\
\text { Alcohol } \\
\text { Use Disorder }\end{array}$ & $\begin{array}{l}\text { The PRIME-MD } \\
\text { Patient Health } \\
\text { Questionnaire } \\
\text { (PHQ) }\end{array}$ & - & $\begin{array}{l}362 \\
200\end{array}$ & $\begin{array}{l}64(17.6 \%) \\
72(36 \%)\end{array}$ & 7 \\
\hline $\begin{array}{l}\text { Erbes, C. R. et } \\
\text { al }(2011) \\
(119)\end{array}$ & USA & $\begin{array}{l}\text { Convenience } \\
\text { Sampling } \\
\text { (CS) }\end{array}$ & $\begin{array}{l}\text { National } \\
\text { Guard/Reserve } \\
\text { veterans returning } \\
\text { from Iraq }\end{array}$ & $\begin{array}{l}\text { The Beck Depression } \\
\text { Inventory } \\
\text { (BDI) }\end{array}$ & 31.60 & 617 & $83(13.5 \%)$ & 7 \\
\hline $\begin{array}{l}\text { Guerra, V. S. } \\
\text { et al (2011) } \\
(120)\end{array}$ & USA & $\begin{array}{l}\text { Convenience } \\
\text { Sampling } \\
\text { (CS) }\end{array}$ & $\begin{array}{l}\text { Veterans in } \\
\text { Operations } \\
\text { Enduring Freedom } \\
\text { and } \\
\text { Iraqi Freedom } \\
\text { (OEF/OIF) }\end{array}$ & $\begin{array}{l}\text { The Beck Depression } \\
\text { Inventory } \\
\text { (BDI) } \\
\text { Beck Scale for Suicide } \\
\text { Ideation Scale for } \\
\text { Suicide Ideation- } \\
\text { Adapted }\end{array}$ & 38.3 & 393 & $88(22.4 \%)$ & 8 \\
\hline
\end{tabular}




\begin{tabular}{|c|c|c|c|c|c|c|c|c|}
\hline $\begin{array}{l}\text { Authors } \\
\text { (Years) }\end{array}$ & Country & $\begin{array}{l}\text { Type of } \\
\text { Sampling } \\
\text { (Type of } \\
\text { Study) }\end{array}$ & Study Population & $\begin{array}{l}\text { Depression } \\
\text { Assessment } \\
\text { Method }\end{array}$ & $\begin{array}{l}\text { Age } \\
\text { (Mean) }\end{array}$ & Sample size & $\begin{array}{l}\text { Prevalence } \\
\text { of } \\
\text { Depression } \\
(\%)\end{array}$ & $\begin{array}{l}\text { NOS } \\
\text { Score }\end{array}$ \\
\hline \multirow{4}{*}{$\begin{array}{l}\text { Jakupcak, M. } \\
\text { et al (2011) } \\
(121)\end{array}$} & \multirow[t]{4}{*}{ USA } & \multirow{4}{*}{$\begin{array}{l}\text { Convenience } \\
\text { Sampling } \\
\text { (CS) }\end{array}$} & \multirow{4}{*}{$\begin{array}{l}\text { Iraq and } \\
\text { Afghanistan War } \\
\text { Veterans in the U.S }\end{array}$} & & \multirow[t]{4}{*}{31} & \multirow[t]{4}{*}{336} & \multirow[t]{4}{*}{$126(37.5 \%)$} & \multirow[t]{4}{*}{7} \\
\hline & & & & \multirow{3}{*}{$\begin{array}{l}\text { Patient Health } \\
\text { Questionnaire } \\
\text { (PHQ) }\end{array}$} & & & & \\
\hline & & & & & & & & \\
\hline & & & & & & & & \\
\hline \multirow{5}{*}{$\begin{array}{l}\text { Kehle, S. M. } \\
\text { et al (2011) } \\
(122)\end{array}$} & \multirow[t]{5}{*}{ USA } & \multirow{5}{*}{$\begin{array}{l}\text { Convenience } \\
\text { Sampling } \\
\text { (CS) }\end{array}$} & $\begin{array}{l}\text { Soldiers from a } \\
\text { National }\end{array}$ & \multirow{5}{*}{$\begin{array}{l}\text { Diagnostic and } \\
\text { Statistical Manual of } \\
\text { Mental Disorders-IV } \\
\text { (DSM-IV) }\end{array}$} & \multirow[t]{5}{*}{31.30} & Total (348) & $51(15 \%)$ & \multirow[t]{5}{*}{7} \\
\hline & & & Guard Brigade & & & Male (304) & $39(13 \%)$ & \\
\hline & & & (BCT) & & & Female (44) & $12(27 \%)$ & \\
\hline & & & $\begin{array}{l}\text { Alcohol use } \\
\text { disorders }\end{array}$ & & & 348 & $45(13 \%)$ & \\
\hline & & & $\begin{array}{l}\text { Substance use } \\
\text { disorders }\end{array}$ & & & 348 & $4(1 \%)$ & \\
\hline \multirow{4}{*}{$\begin{array}{l}\text { Garber, B. G. } \\
\text { et al (2012) } \\
(123)\end{array}$} & \multirow[t]{4}{*}{ Canada } & \multirow{4}{*}{$\begin{array}{l}\text { Convenience } \\
\text { Sampling } \\
\text { (CS) }\end{array}$} & \multirow{4}{*}{$\begin{array}{l}\text { Canadian Forces } \\
\text { Members While on } \\
\text { Deployment to } \\
\text { Afghanistan }\end{array}$} & The PRIME-MD & \multirow[t]{4}{*}{-} & \multirow[t]{4}{*}{1572} & \multirow[t]{4}{*}{$73(4.7 \%)$} & \multirow[t]{4}{*}{5} \\
\hline & & & & Patient Health & & & & \\
\hline & & & & Questionnaire & & & & \\
\hline & & & & (PHQ) & & & & \\
\hline \multirow{3}{*}{$\begin{array}{l}\text { Maguen, S. et } \\
\text { al (2012) } \\
(124)\end{array}$} & \multirow[t]{3}{*}{ USA } & \multirow{3}{*}{$\begin{array}{l}\text { Convenience } \\
\text { Sampling } \\
(\mathrm{Re})\end{array}$} & \multirow{3}{*}{$\begin{array}{l}\text { Iraq and } \\
\text { Afghanistan } \\
\text { Veterans }\end{array}$} & $\begin{array}{l}\text { The Diagnostic and } \\
\text { Statistical }\end{array}$ & \multirow[t]{3}{*}{45} & $\begin{array}{l}\text { Total } \\
(74493)\end{array}$ & $\begin{array}{l}41424(56 \\
\%)\end{array}$ & \multirow[t]{3}{*}{7} \\
\hline & & & & $\begin{array}{l}\text { Manual-Fourth Edition } \\
\text { (DSM-IV) }\end{array}$ & & $\begin{array}{l}\text { Male } \\
(67238)\end{array}$ & $\begin{array}{l}36359(54 \\
\%)\end{array}$ & \\
\hline & & & & & & $\begin{array}{l}\text { Female } \\
(7255)\end{array}$ & $5065(70 \%)$ & \\
\hline $\begin{array}{l}\text { Vasterling, J. } \\
\text { J.et al } \\
\text { (2012)(125) }\end{array}$ & USA & $\begin{array}{l}\text { Convenience } \\
\text { Sampling }\end{array}$ & $\begin{array}{l}\text { Iraq-deployed US } \\
\text { Army soldiers }\end{array}$ & $\begin{array}{l}\text { The Center for } \\
\text { Epidemiological } \\
\text { Studies }\end{array}$ & 25.1 & 760 & $238(31.3 \%)$ & 7 \\
\hline & & (U) & & $\begin{array}{l}\text { Depression Scale } \\
\text { (CES-D) }\end{array}$ & & & & \\
\hline $\begin{array}{l}\text { Cohen, S. I. et } \\
\text { al (2013) }\end{array}$ & USA & $\begin{array}{l}\text { Convenience } \\
\text { Sampling }\end{array}$ & US military & $\begin{array}{l}\text { The Diagnostic and } \\
\text { Statistical }\end{array}$ & - & 93 & $44(47.3 \%)$ & 6 \\
\hline & & (CS) & $\begin{array}{l}\text { from Iraq and } \\
\text { Afghanistan }\end{array}$ & $\begin{array}{l}\text { Manual-Fourth Edition } \\
\text { (DSM-IV) }\end{array}$ & & & & \\
\hline Harbertson, & USA & Convenience & Male Rwanda & The Center for & 30.9 & 1238 & $232(22.1 \%)$ & 7 \\
\hline (2013)(127) & & & military personnel & Studies & & 546 & $129(23.7 \%)$ & \\
\hline & & & $\begin{array}{l}\text { Alcohol Use } \\
\text { Disorder }\end{array}$ & $\begin{array}{l}\text { Depression Scale } \\
\text { (CES-D) }\end{array}$ & & & & \\
\hline $\begin{array}{l}\text { Marshall, B. } \\
\text { D. et al }\end{array}$ & USA & $\begin{array}{l}\text { Convenience } \\
\text { Sampling }\end{array}$ & $\begin{array}{l}\text { Ohio Army } \\
\text { National Guard }\end{array}$ & The PRIME-MD & - & 2117 & $128(6 \%)$ & 7 \\
\hline (2013)(128) & & & Soldiers & Patient Health & & 142 & $17(12 \%)$ & \\
\hline & & & & Questionnaire & & & & \\
\hline & & & Soldiers with HIV & (PHQ) & & & & \\
\hline $\begin{array}{l}\text { Morrow, C. E. } \\
\text { et al (2013) }\end{array}$ & USA & $\begin{array}{l}\text { Convenience } \\
\text { Sampling }\end{array}$ & U.S. Air & The PRIME-MD & 30.35 & 194 & $3(1.6 \%)$ & 5 \\
\hline$(129)$ & & & Force & Patient Health & & & & \\
\hline & & & & Questionnaire & & & & \\
\hline & & & & (PHQ) & & & & \\
\hline
\end{tabular}




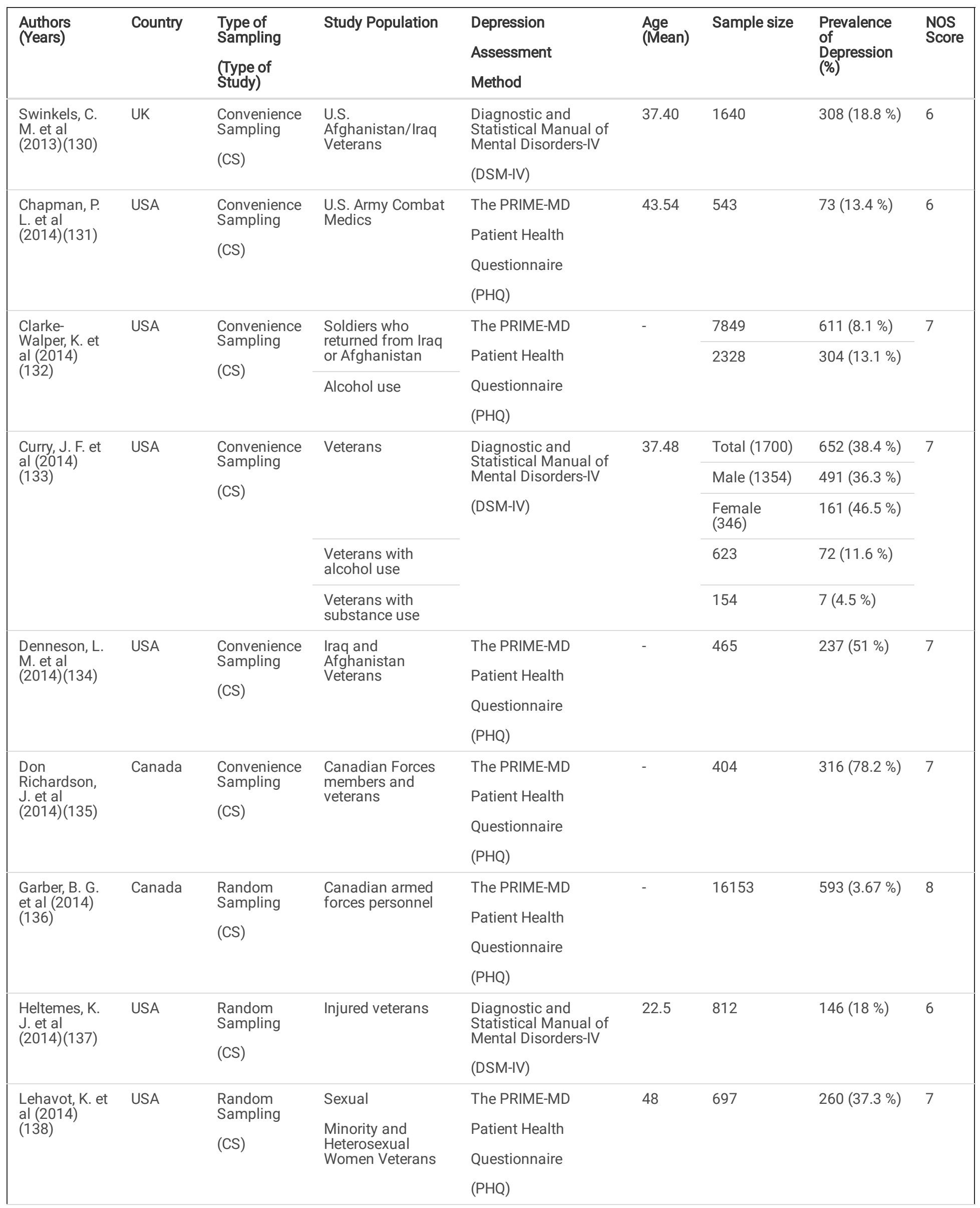




\begin{tabular}{|c|c|c|c|c|c|c|c|c|}
\hline $\begin{array}{l}\text { Authors } \\
\text { (Years) }\end{array}$ & Country & $\begin{array}{l}\text { Type of } \\
\text { Sampling } \\
\text { (Type of } \\
\text { Study) }\end{array}$ & Study Population & $\begin{array}{l}\text { Depression } \\
\text { Assessment } \\
\text { Method }\end{array}$ & $\begin{array}{l}\text { Age } \\
\text { (Mean) }\end{array}$ & Sample size & $\begin{array}{l}\text { Prevalence } \\
\text { of } \\
\text { Depression } \\
(\%)\end{array}$ & $\begin{array}{l}\text { NOS } \\
\text { Score }\end{array}$ \\
\hline $\begin{array}{l}\text { Ramsawh, H. } \\
\text { J. et al } \\
(2014)(139)\end{array}$ & USA & $\begin{array}{l}\text { Convenience } \\
\text { Sampling } \\
\text { (CS) }\end{array}$ & $\begin{array}{l}\text { Active Duty } \\
\text { Military } \\
\text { Personnel }\end{array}$ & $\begin{array}{l}\text { 10-item Center for } \\
\text { Epidemiologic Studies } \\
\text { Depression Scale }\end{array}$ & 35 & 5461 & $1914(35 \%)$ & 8 \\
\hline $\begin{array}{l}\text { Bin Zubair, U. } \\
\text { et al (2015) } \\
(140)\end{array}$ & Pakistan & $\begin{array}{l}\text { Random } \\
\text { Sampling } \\
\text { (CS) }\end{array}$ & $\begin{array}{l}\text { All military recruits } \\
\text { were men and } \\
\text { above the age of } \\
17 \text { years. }\end{array}$ & $\begin{array}{l}\text { The Beck Depression } \\
\text { Inventory } \\
\text { (BDI) }\end{array}$ & 20 & 313 & 159 (50.7 \%) & 7 \\
\hline $\begin{array}{l}\text { Cleveland, S. } \\
\text { D. et al al } \\
(2015)(141)\end{array}$ & USA & $\begin{array}{l}\text { Convenience } \\
\text { Sampling } \\
\text { (CS) }\end{array}$ & $\begin{array}{l}\text { Veterans and } \\
\text { Civilian College } \\
\text { Students }\end{array}$ & $\begin{array}{l}\text { Diagnostic and } \\
\text { Statistical Manual of } \\
\text { Mental Disorders-IV } \\
\text { (DSM-IV) }\end{array}$ & - & 26969 & $\begin{array}{l}7982(30.17 \\
\%)\end{array}$ & 8 \\
\hline $\begin{array}{l}\text { Foote, C. E. et } \\
\text { al (2015) } \\
(142)\end{array}$ & USA & $\begin{array}{l}\text { Random } \\
\text { Sampling } \\
\text { (CS) }\end{array}$ & $\begin{array}{l}\text { Vietnam } \\
\text { veterans }\end{array}$ & $\begin{array}{l}\text { The PRIME-MD } \\
\text { Patient Health } \\
\text { Questionnaire } \\
\text { (PHQ) }\end{array}$ & - & 247 & $44(17.8 \%)$ & 7 \\
\hline $\begin{array}{l}\text { Hamilton, A. } \\
\text { B. et al } \\
\text { (2015) (143) }\end{array}$ & USA & $\begin{array}{l}\text { Random } \\
\text { Sampling } \\
\text { (CS) }\end{array}$ & $\begin{array}{l}\text { Employed Women } \\
\text { Veterans } \\
\text { Unemployed } \\
\text { Women Veterans }\end{array}$ & $\begin{array}{l}\text { The five-question } \\
\text { Mental Health } \\
\text { Inventory (MHI-5) }\end{array}$ & - & $\begin{array}{l}1410 \\
195\end{array}$ & $\begin{array}{l}120(4.1 \%) \\
42(27.3 \%)\end{array}$ & 7 \\
\hline $\begin{array}{l}\text { Hoerster, K. } \\
\text { D. et al } \\
(2015)(144)\end{array}$ & USA & $\begin{array}{l}\text { Random } \\
\text { Sampling } \\
\text { (CS) }\end{array}$ & $\begin{array}{l}\text { Iraq and } \\
\text { Afghanistan } \\
\text { veterans }\end{array}$ & $\begin{array}{l}\text { The PRIME-MD } \\
\text { Patient Health } \\
\text { Questionnaire } \\
\text { (PHQ) }\end{array}$ & 31.3 & 332 & $53(16.3 \%)$ & 7 \\
\hline $\begin{array}{l}\text { Kim, N. Y. et } \\
\text { al (2015) } \\
(145)\end{array}$ & USA & $\begin{array}{l}\text { Convenience } \\
\text { Sampling } \\
\text { (CS) }\end{array}$ & Korean Soldiers & $\begin{array}{l}\text { Scale for suicide } \\
\text { ideation (SSI), } \\
\text { The Beck Depression } \\
\text { Inventory } \\
\text { (BDI) }\end{array}$ & 21.3 & 414 & $21(5 \%)$ & 6 \\
\hline $\begin{array}{l}\text { Lundin, A. et } \\
\text { al (2015) } \\
(146)\end{array}$ & Sweden & $\begin{array}{l}\text { Random } \\
\text { Sampling } \\
\text { (CS) }\end{array}$ & Vietnam veterans & $\begin{array}{l}\text { Diagnostic and } \\
\text { Statistical Manual of } \\
\text { Mental Disorders-IV } \\
\text { (DSM-IV) }\end{array}$ & - & 4251 & $\begin{array}{l}1263(29.7 \\
\%)\end{array}$ & 7 \\
\hline \multirow[t]{2}{*}{$\begin{array}{l}\text { McGuire, A. } \\
\text { et al (2015) } \\
(147)\end{array}$} & \multirow[t]{2}{*}{ UK } & \multirow{2}{*}{$\begin{array}{l}\text { Random } \\
\text { Sampling } \\
\text { (CS) }\end{array}$} & $\begin{array}{l}\text { Australian } \\
\text { Defense Force } \\
\text { (ADF men) }\end{array}$ & $\begin{array}{l}\text { Diagnostic and } \\
\text { Statistical Manual of } \\
\text { Mental Disorders-IV }\end{array}$ & 50 & 4091 & $454(11 \%)$ & \multirow[t]{2}{*}{7} \\
\hline & & & $\begin{array}{l}\text { Department of } \\
\text { Veterans' Affairs } \\
\text { (DVA women) }\end{array}$ & (DSM-IV) & & 4761 & $869(18.3 \%)$ & \\
\hline $\begin{array}{l}\text { Mysliwiec, V. } \\
\text { et al (2015) } \\
(148)\end{array}$ & USA & $\begin{array}{l}\text { Convenience } \\
\text { Sampling } \\
\text { (CS) }\end{array}$ & $\begin{array}{l}\text { U.S. Military } \\
\text { Personnel }\end{array}$ & $\begin{array}{l}\text { Quick Inventory of } \\
\text { Depressive } \\
\text { Symptomatology } \\
\text { (QIDS) }\end{array}$ & 36.2 & 58 & $30(51.7 \%)$ & 7 \\
\hline $\begin{array}{l}\text { Nasioudis, D. } \\
\text { et al (2015) } \\
(149)\end{array}$ & Greece & $\begin{array}{l}\text { Random } \\
\text { Sampling } \\
\text { (CS) }\end{array}$ & $\begin{array}{l}\text { Greek military } \\
\text { medicine } \\
\text { cadets }\end{array}$ & $\begin{array}{l}\text { The Zung Self-report } \\
\text { Depression Scale } \\
\text { (SDS) }\end{array}$ & 19.84 & $\begin{array}{l}\text { Total (146) } \\
\text { Male (91) } \\
\text { Female (55) }\end{array}$ & $\begin{array}{l}57(39 \%) \\
36(39.5 \%) \\
21(38.2 \%)\end{array}$ & 7 \\
\hline
\end{tabular}




\begin{tabular}{|c|c|c|c|c|c|c|c|c|}
\hline $\begin{array}{l}\text { Authors } \\
\text { (Years) }\end{array}$ & Country & $\begin{array}{l}\text { Type of } \\
\text { Sampling } \\
\text { (Type of } \\
\text { Study) }\end{array}$ & Study Population & $\begin{array}{l}\text { Depression } \\
\text { Assessment } \\
\text { Method }\end{array}$ & $\begin{array}{l}\text { Age } \\
\text { (Mean) }\end{array}$ & Sample size & $\begin{array}{l}\text { Prevalence } \\
\text { of } \\
\text { Depression } \\
(\%)\end{array}$ & $\begin{array}{l}\text { NOS } \\
\text { Score }\end{array}$ \\
\hline $\begin{array}{l}\text { Vanderploeg, } \\
\text { R. D. et al } \\
\text { (2015) (150) }\end{array}$ & USA & $\begin{array}{l}\text { Convenience } \\
\text { Sampling } \\
\text { (CS) }\end{array}$ & $\begin{array}{l}\text { Florida National } \\
\text { Guard } \\
\text { Members }\end{array}$ & $\begin{array}{l}\text { The PRIME-MD } \\
\text { Patient Health } \\
\text { Questionnaire } \\
\text { (PHQ) }\end{array}$ & - & 3098 & $63(2 \%)$ & 7 \\
\hline $\begin{array}{l}\text { Fink, D. S. et } \\
\text { al (2016) } \\
(151)\end{array}$ & USA & $\begin{array}{l}\text { Convenience } \\
\text { Sampling } \\
\text { (CS) }\end{array}$ & $\begin{array}{l}\text { U.S. Army National } \\
\text { Guard soldiers }\end{array}$ & $\begin{array}{l}\text { Diagnostic and } \\
\text { Statistical Manual of } \\
\text { Mental Disorders-IV } \\
\text { (DSM-IV) }\end{array}$ & 44 & 671 & $154(23 \%)$ & 8 \\
\hline $\begin{array}{l}\text { Forbes, D. et } \\
\text { al (2016) } \\
(152)\end{array}$ & Australia & $\begin{array}{l}\text { Convenience } \\
\text { Sampling } \\
\text { (CS) }\end{array}$ & $\begin{array}{l}\text { Australian } \\
\text { peacekeepers }\end{array}$ & $\begin{array}{l}\text { Diagnostic and } \\
\text { Statistical Manual of } \\
\text { Mental Disorders-IV } \\
\text { (DSM-IV) }\end{array}$ & 46.5 & 2050 & $201(9.8 \%)$ & 8 \\
\hline $\begin{array}{l}\text { Guloglu, B. et } \\
\text { al (2016) } \\
(153)\end{array}$ & Turkey & $\begin{array}{l}\text { Convenience } \\
\text { Sampling } \\
\text { (CS) }\end{array}$ & $\begin{array}{l}\text { Turkish combat- } \\
\text { injured } \\
\text { non-professional } \\
\text { veterans }\end{array}$ & $\begin{array}{l}\text { The Brief Symptom } \\
\text { Inventory (BSI) }\end{array}$ & 40 & 336 & $55(16.4 \%)$ & 7 \\
\hline $\begin{array}{l}\text { Hardos, J. E. } \\
\text { et al (2016) } \\
(154)\end{array}$ & USA & $\begin{array}{l}\text { Convenience } \\
\text { Sampling } \\
\text { (CS) }\end{array}$ & $\begin{array}{l}\text { Aircraft } \\
\text { Maintenance } \\
\text { Workers }\end{array}$ & $\begin{array}{l}\text { The PRIME-MD } \\
\text { Patient Health } \\
\text { Questionnaire } \\
\text { (PHQ) }\end{array}$ & 29 & 4801 & $\begin{array}{l}1042(21.7 \\
\%)\end{array}$ & 7 \\
\hline $\begin{array}{l}\text { Herberman } \\
\text { Mash, H. B. } \\
\text { et al }(2016) \\
(155)\end{array}$ & USA & $\begin{array}{l}\text { Convenience } \\
\text { Sampling } \\
\text { (CS) }\end{array}$ & $\begin{array}{l}\text { U.S. Army soldiers } \\
\text { U.S. Army soldiers } \\
\text { with alcohol use }\end{array}$ & $\begin{array}{l}\text { The } 10 \text {-item } \\
\text { Center for } \\
\text { Epidemiologic Studies } \\
\text { Depression Scale }\end{array}$ & - & $\begin{array}{l}3813 \\
1210\end{array}$ & $\begin{array}{l}1368(35.8 \\
\%) \\
583(48.18 \\
\%)\end{array}$ & 8 \\
\hline $\begin{array}{l}\text { Monteith, L. } \\
\text { L.et al } \\
(2016)(156)\end{array}$ & USA & $\begin{array}{l}\text { Convenience } \\
\text { Sampling } \\
\text { (CS) }\end{array}$ & Veterans & $\begin{array}{l}\text { Beck Scale for Suicide } \\
\text { Ideation (BSS), }\end{array}$ & 49.6 & Male (310) & $146(47.1 \%)$ & 8 \\
\hline & & & & $\begin{array}{l}\text { Multidimensional } \\
\text { Suicide Inventory-28 } \\
\text { (MSI) Negative Affect } \\
\text { scale }\end{array}$ & & Female (44) & $32(52.3 \%)$ & \\
\hline $\begin{array}{l}\text { Phillips, K. M. } \\
\text { et al (2016) } \\
(157)\end{array}$ & USA & $\begin{array}{l}\text { Convenience } \\
\text { Sampling } \\
\text { (CS) }\end{array}$ & $\begin{array}{l}\text { Iraq- and } \\
\text { Afghanistan-era } \\
\text { Veterans }\end{array}$ & $\begin{array}{l}20- \\
\text { item, self-report Center } \\
\text { for Epidemiological } \\
\text { Studies Depression } \\
\text { Scale (CES-D) }\end{array}$ & 35.1 & 359 & $108(30 \%)$ & 6 \\
\hline $\begin{array}{l}\text { Zamorski, M. } \\
\text { A. et al } \\
(2016)(158)\end{array}$ & Canada & $\begin{array}{l}\text { Convenience } \\
\text { Sampling } \\
\text { (CS) }\end{array}$ & $\begin{array}{l}\text { Canadian Armed } \\
\text { Forces }\end{array}$ & $\begin{array}{l}\text { Diagnostic and } \\
\text { Statistical Manual of } \\
\text { Mental Disorders-IV } \\
\text { (DSM-IV) }\end{array}$ & - & 5120 & 410 (8 \%) & 7 \\
\hline $\begin{array}{l}\text { Boakye, E. A. } \\
\text { et al (2017) } \\
(159)\end{array}$ & USA & $\begin{array}{l}\text { Random } \\
\text { Sampling } \\
\text { (CS) }\end{array}$ & $\begin{array}{l}\text { Veterans } \\
\text { Veterans with } \\
\text { alcohol use }\end{array}$ & Self-Report & 40 & $\begin{array}{l}144 \\
75\end{array}$ & $\begin{array}{l}48(33.3 \%) \\
24(32 \%)\end{array}$ & 7 \\
\hline $\begin{array}{l}\text { Cohen, G. H. } \\
\text { et al (2017) } \\
(160)\end{array}$ & USA & $\begin{array}{l}\text { Convenience } \\
\text { Sampling } \\
\text { (CS) }\end{array}$ & $\begin{array}{l}\text { Army National } \\
\text { Guard Soldiers }\end{array}$ & $\begin{array}{l}\text { The PRIME-MD } \\
\text { Patient Health }\end{array}$ & - & 1582 & $164(10.3 \%)$ & 8 \\
\hline
\end{tabular}




\begin{tabular}{|c|c|c|c|c|c|c|c|c|}
\hline \multirow[t]{4}{*}{$\begin{array}{l}\text { Authors } \\
\text { (Years) }\end{array}$} & \multirow[t]{4}{*}{ Country } & \multirow[t]{4}{*}{$\begin{array}{l}\text { Type of } \\
\text { Sampling } \\
\text { (Type of } \\
\text { Study) }\end{array}$} & Study Population & \multirow{4}{*}{$\begin{array}{l}\text { Depression } \\
\text { Assessment } \\
\text { Method } \\
\text { Questionnaire } \\
\text { (PHQ), } \\
\text { The PHQ-9 Item }\end{array}$} & \multirow[t]{4}{*}{$\begin{array}{l}\text { Age } \\
\text { (Mean) }\end{array}$} & \multirow[t]{2}{*}{ Sample size } & $\begin{array}{l}\text { Prevalence } \\
\text { of } \\
\text { Depression } \\
\text { (\%) }\end{array}$ & $\begin{array}{l}\text { NOS } \\
\text { Score }\end{array}$ \\
\hline & & & & & & & & \\
\hline & & & Army National & & & 93 & $27(29 \%)$ & \\
\hline & & & With Alconol use & & & & & \\
\hline \multirow{4}{*}{$\begin{array}{l}\text { Gradus, J. L. } \\
\text { et al (2017) } \\
(161)\end{array}$} & \multirow[t]{4}{*}{ USA } & \multirow{4}{*}{$\begin{array}{l}\text { Random } \\
\text { Sampling } \\
\text { (CS) }\end{array}$} & \multirow{4}{*}{$\begin{array}{l}\text { Veterans of the } \\
\text { Iraq and } \\
\text { Afghanistan Wars }\end{array}$} & \multirow{2}{*}{$\begin{array}{l}20- \\
\text { item, self-report Center } \\
\text { for Epidemiological } \\
\text { Studies Depression } \\
\text { Scale (CES-D), }\end{array}$} & \multirow[t]{4}{*}{34} & Total (2244) & $712(31.7 \%)$ & \multirow[t]{4}{*}{7} \\
\hline & & & & & & Male (1062) & $314(29.5 \%)$ & \\
\hline & & & & $\begin{array}{l}\text { The 4-item Suicidal } \\
\text { Behaviors }\end{array}$ & & $\begin{array}{l}\text { Female } \\
\text { (1099) }\end{array}$ & $398(36.3 \%)$ & \\
\hline & & & & $\begin{array}{l}\text { Questionnaire-Short } \\
\text { Form (SBQ-SF) }\end{array}$ & & & & \\
\hline \multirow{4}{*}{$\begin{array}{l}\text { Packnett, E. } \\
\text { R. et al } \\
(2017)(162)\end{array}$} & \multirow[t]{4}{*}{ USA } & \multirow{4}{*}{$\begin{array}{l}\text { Convenience } \\
\text { Sampling } \\
\text { (CS) }\end{array}$} & Army & \multirow{4}{*}{$\begin{array}{l}\text { Diagnostic and } \\
\text { Statistical Manual of } \\
\text { Mental Disorders-IV } \\
\text { (DSM-IV) }\end{array}$} & \multirow[t]{4}{*}{-} & 34487 & $1777(5.1 \%)$ & \multirow[t]{4}{*}{8} \\
\hline & & & Navy & & & 6602 & $263(4 \%)$ & \\
\hline & & & Marine Corps & & & 8428 & $113(1.3 \%)$ & \\
\hline & & & Air Force & & & 9510 & $729(7.6 \%)$ & \\
\hline $\begin{array}{l}\text { Weeks, M. et } \\
\text { al (2017) } \\
(163)\end{array}$ & Canada & $\begin{array}{l}\text { Convenience } \\
\text { Sampling } \\
\text { (CS) }\end{array}$ & $\begin{array}{l}\text { Canadian Military } \\
\text { and Civilian } \\
\text { Populations }\end{array}$ & $\begin{array}{l}\text { Diagnostic and } \\
\text { Statistical Manual of } \\
\text { Mental Disorders-IV } \\
\text { (DSM-IV) }\end{array}$ & 35 & 6696 & $536(8 \%)$ & 8 \\
\hline $\begin{array}{l}\text { Bartlett, B. A. } \\
\text { et al (2018) } \\
(164)\end{array}$ & USA & $\begin{array}{l}\text { Convenience } \\
\text { Sampling } \\
\text { (CS) }\end{array}$ & Military veterans & $\begin{array}{l}20- \\
\text { item, self-report Center } \\
\text { for Epidemiological } \\
\text { Studies Depression } \\
\text { Scale (CES-D) }\end{array}$ & 38.40 & 910 & $75(9.8 \%)$ & 6 \\
\hline $\begin{array}{l}\text { Blakey, S. M. } \\
\text { et al (2018) } \\
(165)\end{array}$ & USA & $\begin{array}{l}\text { Convenience } \\
\text { Sampling } \\
\text { (CS) }\end{array}$ & $\begin{array}{l}\text { U.S. veterans, } \\
\text { active duty } \\
\text { personnel, and } \\
\text { National } \\
\text { Guard and Reserve } \\
\text { members }\end{array}$ & $\begin{array}{l}\text { Diagnostic and } \\
\text { Statistical Manual of } \\
\text { Mental Disorders-IV } \\
\text { (DSM-IV) }\end{array}$ & 37.8 & 667 & $169(25.3 \%)$ & 7 \\
\hline $\begin{array}{l}\text { Boulos, D. et } \\
\text { al (2018) }\end{array}$ & Canada & $\begin{array}{l}\text { Random } \\
\text { Sampling } \\
\text { (CS) }\end{array}$ & $\begin{array}{l}\text { Regular Force } \\
\text { personnel }\end{array}$ & $\begin{array}{l}\text { Diagnostic and } \\
\text { Statistical Manual of }\end{array}$ & - & 3385 & $129(3.8 \%)$ & 7 \\
\hline & & & $\begin{array}{l}\text { Reserve Force } \\
\text { personnel }\end{array}$ & & & 1469 & $55(3.7 \%)$ & \\
\hline (166) & & & & Mental Disorders-IV & & & & \\
\hline & & & & (DSM-IV) & & & & \\
\hline $\begin{array}{l}\text { Dillon, K. H. } \\
\text { et al (2018) } \\
(167)\end{array}$ & USA & $\begin{array}{l}\text { Convenience } \\
\text { Sampling } \\
\text { (CS) }\end{array}$ & $\begin{array}{l}\text { Iraq/Afghanistan- } \\
\text { era veterans }\end{array}$ & $\begin{array}{l}\text { The Beck Scale for } \\
\text { Suicide Ideation } \\
\text { (BSS), The Structured } \\
\text { Clinical Interview for } \\
\text { DSM-IV-TR (SCID) }\end{array}$ & - & 3238 & $\begin{array}{l}1315 \\
(40.6 \%)\end{array}$ & 7 \\
\hline $\begin{array}{l}\text { Don } \\
\text { Richardson }\end{array}$ & Canada & $\begin{array}{l}\text { Convenience } \\
\text { Sampling }\end{array}$ & $\begin{array}{l}\text { Canadian Armed } \\
\text { Forces members }\end{array}$ & The PRIME-MD & 44.6 & 522 & $413(79.1 \%)$ & 7 \\
\hline $\begin{array}{l}\text { J. et al } \\
(0018)(168)\end{array}$ & & & and veterans & Patient Health & & & & \\
\hline & & & & Questionnaire & & & & \\
\hline & & & & (PHQ) & & & & \\
\hline
\end{tabular}




\begin{tabular}{|c|c|c|c|c|c|c|c|c|}
\hline $\begin{array}{l}\text { Authors } \\
\text { (Years) }\end{array}$ & Country & $\begin{array}{l}\text { Type of } \\
\text { Sampling } \\
\text { (Type of } \\
\text { Study) }\end{array}$ & Study Population & $\begin{array}{l}\text { Depression } \\
\text { Assessment } \\
\text { Method }\end{array}$ & $\begin{array}{l}\text { Age } \\
\text { (Mean) }\end{array}$ & Sample size & $\begin{array}{l}\text { Prevalence } \\
\text { of } \\
\text { Depression } \\
(\%)\end{array}$ & $\begin{array}{l}\text { NOS } \\
\text { Score }\end{array}$ \\
\hline $\begin{array}{l}\text { Elbogen, E. B. } \\
\text { et al (2018) } \\
(169)\end{array}$ & USA & $\begin{array}{l}\text { Convenience } \\
\text { Sampling } \\
\text { (CS) }\end{array}$ & $\begin{array}{l}\text { Iraq/Afghanistan- } \\
\text { era veterans }\end{array}$ & $\begin{array}{l}\text { Diagnostic and } \\
\text { Statistical Manual of } \\
\text { Mental Disorders-IV } \\
\text { (DSM-IV) }\end{array}$ & 34.9 & 1172 & 375 (32 \%) & 6 \\
\hline $\begin{array}{l}\text { Hourani, L. L. } \\
\text { et al (2018) } \\
(170)\end{array}$ & USA & $\begin{array}{l}\text { Convenience } \\
\text { Sampling } \\
\text { (CS) }\end{array}$ & $\begin{array}{l}\text { Active duty } \\
\text { military personnel }\end{array}$ & $\begin{array}{l}\text { The PRIME-MD } \\
\text { Patient Health } \\
\text { Questionnaire } \\
\text { (PHQ), Checklist }\end{array}$ & - & 947 & $115(15.4 \%)$ & 7 \\
\hline $\begin{array}{l}\text { Kizilhan, J. I. } \\
\text { et al (2018) } \\
(171)\end{array}$ & Iraq & $\begin{array}{l}\text { Convenience } \\
\text { Sampling } \\
\text { (CS) }\end{array}$ & Child soldiers & $\begin{array}{l}\text { Diagnostic and } \\
\text { Statistical Manual of } \\
\text { Mental Disorders-IV } \\
\text { (DSM-IV) }\end{array}$ & 12.6 & 81 & $37(45.6 \%)$ & 6 \\
\hline $\begin{array}{l}\text { McDonald, S. } \\
\text { D. et al } \\
\text { (2018)(172) }\end{array}$ & USA & $\begin{array}{l}\text { Convenience } \\
\text { Sampling } \\
\text { (CS) }\end{array}$ & $\begin{array}{l}\text { U.S. } \\
\text { Department of } \\
\text { Veterans Affairs } \\
\text { outpatients }\end{array}$ & $\begin{array}{l}\text { Diagnostic and } \\
\text { Statistical Manual of } \\
\text { Mental Disorders-IV } \\
\text { (DSM-IV) }\end{array}$ & 58.1 & 280 & $53(19 \%)$ & 7 \\
\hline $\begin{array}{l}\text { Stefanovics, } \\
\text { E. A. et al } \\
\text { (2018)(173) }\end{array}$ & USA & $\begin{array}{l}\text { Convenience } \\
\text { Sampling } \\
\text { (CS) }\end{array}$ & US Veterans & $\begin{array}{l}\text { The Patient Health } \\
\text { Questionnaire-4 }\end{array}$ & 59 & 3122 & 209 (6.7 \%) & 7 \\
\hline $\begin{array}{l}\text { Vun, E. et al } \\
(2018)(174)\end{array}$ & Canada & $\begin{array}{l}\text { Convenience } \\
\text { Sampling } \\
\text { (CS) }\end{array}$ & $\begin{array}{l}\text { Canadian Armed } \\
\text { Forces active } \\
\text { personnel }\end{array}$ & $\begin{array}{l}\text { Diagnostic and } \\
\text { Statistical Manual of } \\
\text { Mental Disorders-IV } \\
\text { (DSM-IV) }\end{array}$ & 35.4 & 6696 & $517(8 \%)$ & 8 \\
\hline $\begin{array}{l}\text { Waitzkin, H. } \\
\text { et al }(2018) \\
(175)\end{array}$ & USA & $\begin{array}{l}\text { Convenience } \\
\text { Sampling } \\
\text { (CS) }\end{array}$ & Military Personnel & $\begin{array}{l}\text { The PRIME-MD } \\
\text { Patient Health } \\
\text { Questionnaire } \\
\text { (PHQ) }\end{array}$ & - & 198 & $143(72 \%)$ & 7 \\
\hline $\begin{array}{l}\text { Byrne, S. P. et } \\
\text { al (2019) } \\
(176)\end{array}$ & USA & $\begin{array}{l}\text { Convenience } \\
\text { Sampling } \\
\text { (CS) }\end{array}$ & $\begin{array}{l}\text { U.S. military } \\
\text { veterans }\end{array}$ & $\begin{array}{l}\text { The PRIME-MD } \\
\text { Patient Health } \\
\text { Questionnaire } \\
(\mathrm{PHQ})\end{array}$ & 53.4 & 158 & $62(34.7 \%)$ & 7 \\
\hline $\begin{array}{l}\text { Carney, B. et } \\
\text { al (2019) } \\
(177)\end{array}$ & USA & $\begin{array}{l}\text { Random } \\
\text { Sampling } \\
\text { (CS) }\end{array}$ & $\begin{array}{l}\text { US Military } \\
\text { population with } \\
\text { HIV } \\
\text { infection }\end{array}$ & $\begin{array}{l}20- \\
\text { item, self-report Center } \\
\text { for Epidemiological } \\
\text { Studies Depression } \\
\text { Scale (CES-D) }\end{array}$ & 32 & 662 & $114(17.2 \%)$ & 8 \\
\hline $\begin{array}{l}\text { Jones, N. et } \\
\text { al (2019) } \\
(178)\end{array}$ & UK & $\begin{array}{l}\text { Random } \\
\text { Sampling } \\
\text { (CS) }\end{array}$ & UK Armed Forces & The PRIME-MD & - & $\begin{array}{l}\text { Total (1448) } \\
\text { Male (1229) }\end{array}$ & $\begin{array}{l}110(7.6 \%) \\
93(7.7 \%)\end{array}$ & 6 \\
\hline & & & & $\begin{array}{l}\text { Patient Health } \\
\text { Questionnaire } \\
\text { (PHQ) }\end{array}$ & & $\begin{array}{l}\text { Female } \\
\text { (219) }\end{array}$ & $17(7.9 \%)$ & \\
\hline $\begin{array}{l}\text { Lucas, C. L. } \\
\text { et al (2019) } \\
(179)\end{array}$ & USA & $\begin{array}{l}\text { Convenience } \\
\text { Sampling } \\
\text { (CS) }\end{array}$ & Military Personnel & $\begin{array}{l}\text { The PRIME-MD } \\
\text { Patient Health } \\
\text { Questionnaire }\end{array}$ & - & $\begin{array}{l}\text { Total (1980) } \\
\text { Male (1665) }\end{array}$ & $\begin{array}{l}660(37.9 \%) \\
530(36.2 \%)\end{array}$ & 7 \\
\hline
\end{tabular}




\begin{tabular}{|c|c|c|c|c|c|c|c|c|}
\hline $\begin{array}{l}\text { Authors } \\
\text { (Years) }\end{array}$ & Country & $\begin{array}{l}\text { Type of } \\
\text { Sampling } \\
\text { (Type of } \\
\text { Study) }\end{array}$ & Study Population & $\begin{array}{l}\text { Bepression } \\
\text { Assessment } \\
\text { Method }\end{array}$ & $\begin{array}{l}\text { Age } \\
\text { (Mean) }\end{array}$ & Sample size & $\begin{array}{l}\text { Prevalence } \\
\text { of } \\
\text { Depression } \\
(\%)\end{array}$ & $\begin{array}{l}\text { NOS } \\
\text { Score }\end{array}$ \\
\hline & & & & & & $\begin{array}{l}\text { Female } \\
(315)\end{array}$ & $130(46.8 \%)$ & \\
\hline \multirow[t]{2}{*}{$\begin{array}{l}\text { Nichter, B. et } \\
\text { al (2019) } \\
(180)\end{array}$} & \multirow[t]{2}{*}{ USA } & \multirow[t]{2}{*}{$\begin{array}{l}\text { Random } \\
\text { Sampling } \\
\text { (CS) }\end{array}$} & \multirow[t]{2}{*}{$\begin{array}{l}\text { U.S. } \\
\text { veteran population }\end{array}$} & $\begin{array}{l}\text { The Patient Health } \\
\text { Questionnaire-4 (PHQ- } \\
\text { 4), The Patient Health }\end{array}$ & \multirow[t]{2}{*}{60.3} & \multirow[t]{2}{*}{2732} & \multirow[t]{2}{*}{$201(7.3 \%)$} & \multirow[t]{2}{*}{9} \\
\hline & & & & $\begin{array}{l}\text { Questionnaire-9 (PHQ- } \\
\text { 9) }\end{array}$ & & & & \\
\hline \multirow{2}{*}{$\begin{array}{l}\text { Start, A. R. et } \\
\text { al (2019) } \\
(181)\end{array}$} & \multirow[t]{2}{*}{ USA } & \multirow{2}{*}{$\begin{array}{l}\text { Convenience } \\
\text { Sampling } \\
\text { (CS) }\end{array}$} & \multirow[t]{2}{*}{ Military Personnel } & The Patient Health & \multirow[t]{2}{*}{-} & \multirow[t]{2}{*}{944} & \multirow[t]{2}{*}{$72(7.6 \%)$} & \multirow[t]{2}{*}{7} \\
\hline & & & & $\begin{array}{l}\text { Questionnaire-9 (PHQ- } \\
\text { 9) }\end{array}$ & & & & \\
\hline \multirow[t]{2}{*}{$\begin{array}{l}\text { Blosnich, J. } \\
\text { R. et al } \\
(2020)(182)\end{array}$} & \multirow[t]{2}{*}{ USA } & \multirow{2}{*}{$\begin{array}{l}\text { Random } \\
\text { Sampling } \\
\text { (CS) }\end{array}$} & \multirow[t]{2}{*}{ Military Veterans } & $\begin{array}{l}\text { Diagnostic and } \\
\text { Statistical Manual of } \\
\text { Mental Disorders-IV }\end{array}$ & \multirow[t]{2}{*}{-} & \multirow[t]{2}{*}{293872} & \multirow[t]{2}{*}{$\begin{array}{l}45391(15.4 \\
\%)\end{array}$} & \multirow[t]{2}{*}{9} \\
\hline & & & & (DSM-IV) & & & & \\
\hline \multirow{2}{*}{$\begin{array}{l}\text { Forys- } \\
\text { Donahue, K. } \\
\text { L.et al } \\
(2020)(183)\end{array}$} & \multirow[t]{2}{*}{ USA } & \multirow{2}{*}{$\begin{array}{l}\text { Random } \\
\text { Sampling } \\
\text { (CS) }\end{array}$} & \multirow{2}{*}{$\begin{array}{l}\text { US Army } \\
\text { population }\end{array}$} & The Patient Health & \multirow[t]{2}{*}{-} & \multirow[t]{2}{*}{7043} & \multirow[t]{2}{*}{$774(11 \%)$} & \multirow[t]{2}{*}{6} \\
\hline & & & & $\begin{array}{l}\text { Questionnaire-9 (PHQ- } \\
\text { 9) }\end{array}$ & & & & \\
\hline Gjerstad, C. & Norway & $\begin{array}{l}\text { Convenience } \\
\text { Sampling }\end{array}$ & Norwegian & The Hospital Anxiety & 30 & 10450 & $417(4 \%)$ & 8 \\
\hline (2020)(184) & & & Peacekeepers & $(\mathrm{HADS})$ & & & & \\
\hline Groll, D. L. et & Canada & Convenience & Canadian military & The Patient Health & - & 477 & $61(12.8 \%)$ & 8 \\
\hline (185) & & & & $\begin{array}{l}\text { Questionnaire-9 (PHQ- } \\
\text { 9) }\end{array}$ & & & & \\
\hline Gross, G. M. & USA & Random & U.S. & The Patient Health & 35 & Total (650) & $306(47 \%)$ & 7 \\
\hline & & & veteran population & Questionnaire-9 (PHQ- & & Male (498) & $192(38.6 \%)$ & \\
\hline & & & & & & $\begin{array}{l}\text { Female } \\
(352)\end{array}$ & $114(32.4 \%)$ & \\
\hline $\begin{array}{l}\text { Shim, E. J. et } \\
\text { al (2020) }\end{array}$ & Korea & $\begin{array}{l}\text { Random } \\
\text { Sampling }\end{array}$ & $\begin{array}{l}\text { Korean military } \\
\text { population }\end{array}$ & & 50.6 & 1937 & $162(8.4 \%)$ & 8 \\
\hline & & (CS) & & $\begin{array}{l}\text { International } \\
\text { Neuropsychiatric } \\
\text { Interview Plus (MINI- } \\
\text { Plus), The Patient } \\
\text { Health }\end{array}$ & & & & \\
\hline & & & & $\begin{array}{l}\text { Questionnaire-9 (PHQ- } \\
\text { 9) }\end{array}$ & & & & \\
\hline $\begin{array}{l}\text { Smigelsky, } \\
\text { M. A. et al } \\
(2020)(188)\end{array}$ & USA & $\begin{array}{l}\text { Convenience } \\
\text { Sampling }\end{array}$ & $\begin{array}{l}\text { U.S. military } \\
\text { population }\end{array}$ & $\begin{array}{l}\text { Diagnostic and } \\
\text { Statistical Manual of } \\
\text { Mental Disorders-IV }\end{array}$ & 37.6 & 1002 & $210(21 \%)$ & 6 \\
\hline & & & & (DSM-IV) & & & & \\
\hline Smith, L. M. & USA & Convenience & U.S. Air Force & The Patient Health & - & 85 & $20(23.5 \%)$ & 5 \\
\hline (189) & & & Training & $\begin{array}{l}\text { Questionnaire-9 (PHQ- } \\
\text { 9) }\end{array}$ & & & & \\
\hline $\begin{array}{l}\text { Stefanovics, } \\
\text { E. A. et al } \\
(2020)(190)\end{array}$ & USA & $\begin{array}{l}\text { Convenience } \\
\text { Sampling } \\
\text { (CS) }\end{array}$ & $\begin{array}{l}\text { U.S. Military } \\
\text { Veterans }\end{array}$ & $\begin{array}{l}\text { The Mini International } \\
\text { Neuropsychiatric } \\
\text { Interview (MINI), The } \\
\text { Patient Health }\end{array}$ & 55 & 1308 & $340(30 \%)$ & 5 \\
\hline & & & & $\begin{array}{l}\text { Questionnaire-9 (PHQ- } \\
\text { 9) }\end{array}$ & & & & \\
\hline
\end{tabular}




\begin{tabular}{|c|c|c|c|c|c|c|c|c|}
\hline $\begin{array}{l}\text { Authors } \\
\text { (Years) }\end{array}$ & Country & $\begin{array}{l}\text { Type of } \\
\text { Sampling } \\
\text { (Type of } \\
\text { Study) }\end{array}$ & Study Population & $\begin{array}{l}\text { Depression } \\
\text { Assessment } \\
\text { Method }\end{array}$ & $\begin{array}{l}\text { Age } \\
\text { (Mean) }\end{array}$ & Sample size & $\begin{array}{l}\text { Prevalence } \\
\text { of } \\
\text { Depression } \\
(\%)\end{array}$ & $\begin{array}{l}\text { NOS } \\
\text { Score }\end{array}$ \\
\hline $\begin{array}{l}\text { Taillieu, T. L. } \\
\text { et al (2020) } \\
(191)\end{array}$ & Canada & $\begin{array}{l}\text { Convenience } \\
\text { Sampling } \\
\text { (CS) }\end{array}$ & $\begin{array}{l}\text { Canadian Armed } \\
\text { Forces }\end{array}$ & $\begin{array}{l}\text { Diagnostic and } \\
\text { Statistical Manual of } \\
\text { Mental Disorders-IV } \\
\text { (DSM-IV) }\end{array}$ & - & 6447 & $1006(15.6 \%)$ & 5 \\
\hline $\begin{array}{l}\text { Wang, J. et al } \\
(2020)(192)\end{array}$ & USA & $\begin{array}{l}\text { Convenience } \\
\text { Sampling } \\
\text { (CS) }\end{array}$ & $\begin{array}{l}\text { U.S. } \\
\text { Reserve and } \\
\text { National Guard } \\
\text { Personnel }\end{array}$ & $\begin{array}{l}\text { The Patient Health } \\
\text { Questionnaire-9 (PHQ- } \\
\text { 9) }\end{array}$ & 34.4 & 3503 & $86(2.5 \%)$ & 6 \\
\hline $\begin{array}{l}\text { Ursano, R. J. } \\
\text { et al (2020) } \\
\text { (193) }\end{array}$ & USA & $\begin{array}{l}\text { Convenience } \\
\text { Sampling } \\
\text { (CS) }\end{array}$ & $\begin{array}{l}\text { US Army Soldiers } \\
\text { During } \\
\text { Deployment } \\
\text { in Afghanistan }\end{array}$ & $\begin{array}{l}\text { Diagnostic and } \\
\text { Statistical Manual of } \\
\text { Mental Disorders-IV } \\
\text { (DSM-IV) }\end{array}$ & - & 3957 & $173(4.1 \%)$ & 7 \\
\hline $\begin{array}{l}\text { Yeom, C. W. } \\
\text { et al }(2020) \\
(194)\end{array}$ & Korea & $\begin{array}{l}\text { Convenience } \\
\text { Sampling } \\
\text { (CS) }\end{array}$ & $\begin{array}{l}\text { Korean military } \\
\text { personal }\end{array}$ & $\begin{array}{l}\text { The Mini } \\
\text { International } \\
\text { Neuropsychiatric } \\
\text { Interview Plus } \\
\text { (MINI-Plus Suicidality } \\
\text { module), The Patient } \\
\text { Health } \\
\text { Questionnaire-9 (PHQ- } \\
\text { 9) }\end{array}$ & 21.4 & 480 & $27(5.6 \%)$ & 6 \\
\hline
\end{tabular}


Table 2

The study characteristics of included studies about suicide attempted and thought

\begin{tabular}{|c|c|c|c|c|c|c|c|c|c|}
\hline \multirow[t]{2}{*}{$\begin{array}{l}\text { Authors } \\
\text { (Years) }\end{array}$} & \multirow[t]{2}{*}{ Country } & \multirow{2}{*}{$\begin{array}{l}\text { Type of } \\
\text { Sampling } \\
\text { (Type of } \\
\text { Study) }\end{array}$} & \multirow[t]{2}{*}{ Study Population } & \multirow{2}{*}{$\begin{array}{l}\text { Depression } \\
\text { Assessment } \\
\text { Method }\end{array}$} & \multirow[t]{2}{*}{$\begin{array}{l}\text { Age } \\
\text { (Mean) }\end{array}$} & \multirow[t]{2}{*}{$\begin{array}{l}\text { Sample } \\
\text { size }\end{array}$} & \multicolumn{2}{|c|}{$\begin{array}{l}\text { Prevalence of Suicide } \\
(\%)\end{array}$} & \multirow[t]{2}{*}{$\begin{array}{l}\text { NOS } \\
\text { Score }\end{array}$} \\
\hline & & & & & & & Attempts & Thoughts & \\
\hline $\begin{array}{l}\text { Helzer, J. E. et } \\
\text { al (1976) (66) }\end{array}$ & USA & $\begin{array}{l}\text { Random } \\
\text { Sampling } \\
\text { (CS) }\end{array}$ & Army men & $\begin{array}{l}\text { Clinical } \\
\text { Symptoms } \\
\text { (Interviews) }\end{array}$ & - & 470 & NR & $42(9 \%)$ & 7 \\
\hline $\begin{array}{l}\text { Bohnker, B. et } \\
\text { al (1992) } \\
(195)\end{array}$ & USA & $\begin{array}{l}\text { Random } \\
\text { Sampling } \\
\text { (CS) }\end{array}$ & $\begin{array}{l}\text { Aircraft Carrier } \\
\text { (men) }\end{array}$ & NR & - & 150 & $\begin{array}{l}102(68 \\
\%)\end{array}$ & NR & 6 \\
\hline $\begin{array}{l}\text { Brown, G. R. et } \\
\text { al (1993) (70) }\end{array}$ & USA & $\begin{array}{l}\text { Random } \\
\text { Sampling } \\
\text { (CS) }\end{array}$ & $\begin{array}{l}\text { Air Forces men } \\
\text { with HIV }\end{array}$ & $\begin{array}{l}\text { Structured } \\
\text { Interview Guide } \\
\text { for the Hamilton } \\
\text { Anxiety and } \\
\text { Depression } \\
\text { Scales (SIGH-AD) }\end{array}$ & 35 & 442 & $\begin{array}{l}24(5.4 \\
\%)\end{array}$ & NR & 8 \\
\hline $\begin{array}{l}\text { Lish, J. D. et al } \\
(1996)(74)\end{array}$ & USA & $\begin{array}{l}\text { Random } \\
\text { Sampling } \\
\text { (CS) }\end{array}$ & $\begin{array}{l}\text { Army men and } \\
\text { women }\end{array}$ & $\begin{array}{l}\text { Brief self-report } \\
\text { questionnaire } \\
\text { (SCRENNER) }\end{array}$ & 21.2 & 669 & NR & 51 (7.62 \%) & 7 \\
\hline $\begin{array}{l}\text { Benda, B. B. } \\
(2003) \text { (196) }\end{array}$ & USA & $\begin{array}{l}\text { Convenience } \\
\text { Sampling } \\
\text { (CS) }\end{array}$ & $\begin{array}{l}\text { Veterans } \\
\text { Who Abuse } \\
\text { Substances }\end{array}$ & $\begin{array}{l}\text { Multi-Problem } \\
\text { Screening } \\
\text { Inventory (MPSI) }\end{array}$ & 50.3 & 600 & $\begin{array}{l}240(40 \\
\%)\end{array}$ & $\begin{array}{l}184(30.7 \\
\%)\end{array}$ & 7 \\
\hline $\begin{array}{l}\text { Ritchie, E. C. } \\
\text { et al (2003) } \\
(197)\end{array}$ & USA & $\begin{array}{l}\text { Convenience } \\
\text { Sampling } \\
\text { (CS) }\end{array}$ & $\begin{array}{l}\text { Men and Women } \\
\text { in the Navy and } \\
\text { Marine Corps }\end{array}$ & - & 43 & 100 & $\begin{array}{l}54(54 \\
\%)\end{array}$ & NR & 5 \\
\hline $\begin{array}{l}\text { Benda, B. B. et } \\
\text { al (2005)(198) }\end{array}$ & USA & $\begin{array}{l}\text { Convenience } \\
\text { Sampling } \\
\text { (CS) }\end{array}$ & $\begin{array}{l}\text { Veterans } \\
\text { Who Abuse } \\
\text { Substances }\end{array}$ & $\begin{array}{l}\text { The Multi- } \\
\text { Problem } \\
\text { Screening } \\
\text { Inventory (MPSI) }\end{array}$ & 40.3 & 625 & $\begin{array}{l}197 \\
(31.5 \%)\end{array}$ & $\begin{array}{l}291(46.5 \\
\%)\end{array}$ & 6 \\
\hline $\begin{array}{l}\text { Hoge, C. W. et } \\
\text { al (2006)(101) }\end{array}$ & USA & $\begin{array}{l}\text { Random } \\
\text { Sampling } \\
\text { (CS) }\end{array}$ & $\begin{array}{l}\text { Army soldiers } \\
\text { and Marines }\end{array}$ & $\begin{array}{l}\text { The PRIME-MD } \\
\text { Patient Health } \\
\text { Questionnaire } \\
\text { (PHQ) }\end{array}$ & 31.2 & 303905 & NR & $\begin{array}{l}3501(1.15 \\
\%)\end{array}$ & 8 \\
\hline $\begin{array}{l}\text { Dove, M. B. et } \\
\text { al }(2007)(104)\end{array}$ & USA & $\begin{array}{l}\text { Convenience } \\
\text { Sampling } \\
\text { (CS) }\end{array}$ & $\begin{array}{l}\text { Women Entering } \\
\text { a Military } \\
\text { Substance } \\
\text { Use Disorder }\end{array}$ & $\begin{array}{l}\text { Depression } \\
\text { Checklist }\end{array}$ & - & 86 & NR & $15(17.4 \%)$ & 5 \\
\hline \multirow[t]{3}{*}{$\begin{array}{l}\text { Kline, A. et al } \\
(2009)(109)\end{array}$} & USA & $\begin{array}{l}\text { Convenience } \\
\text { Sampling } \\
\text { (CS) }\end{array}$ & $\begin{array}{l}\text { Vietnam veterans } \\
\text { with Substance } \\
\text { Use Disorder }\end{array}$ & $\begin{array}{l}\text { SCID DSM-IV } \\
\text { Diagnoses }\end{array}$ & 55.20 & 82 & $\begin{array}{l}23(27.8 \\
\%)\end{array}$ & $5(6.1 \%)$ & 8 \\
\hline & & & $\begin{array}{l}\text { Post-Vietnam } \\
\text { veterans with } \\
\text { Substance } \\
\text { Use Disorder }\end{array}$ & & 46.76 & 236 & $\begin{array}{l}63(26.8 \\
\%)\end{array}$ & $16(6.8 \%)$ & \\
\hline & & & $\begin{array}{l}\text { Persian Gulf } \\
\text { veterans with } \\
\text { Substance } \\
\text { Use Disorder }\end{array}$ & & 34 & 55 & $\begin{array}{l}9(15.4 \\
\%)\end{array}$ & $5(9.1 \%)$ & \\
\hline $\begin{array}{l}\text { Rehn, L. M. et } \\
\text { al (2009)(110) }\end{array}$ & Finland & $\begin{array}{l}\text { Convenience } \\
\text { Sampling } \\
\text { (CS) }\end{array}$ & $\begin{array}{l}\text { Male Finnish } \\
\text { military } \\
\text { conscripts }\end{array}$ & $\begin{array}{l}\text { The Beck } \\
\text { Depression } \\
\text { Inventory } \\
\text { (BDI) }\end{array}$ & 20 & 126 & NR & $9(7.1 \%)$ & 6 \\
\hline
\end{tabular}




\begin{tabular}{|c|c|c|c|c|c|c|c|c|c|}
\hline \multirow[t]{2}{*}{$\begin{array}{l}\text { Authors } \\
\text { (Years) }\end{array}$} & \multirow[t]{2}{*}{ Country } & \multirow{2}{*}{$\begin{array}{l}\text { Type of } \\
\text { Sampling } \\
\text { (Type of } \\
\text { Study) }\end{array}$} & \multirow[t]{2}{*}{ Study Population } & \multirow{2}{*}{$\begin{array}{l}\text { Depression } \\
\text { Assessment } \\
\text { Method }\end{array}$} & \multirow[t]{2}{*}{$\begin{array}{l}\text { Age } \\
\text { (Mean) }\end{array}$} & \multirow[t]{2}{*}{$\begin{array}{l}\text { Sample } \\
\text { size }\end{array}$} & \multicolumn{2}{|c|}{$\begin{array}{l}\text { Prevalence of Suicide } \\
(\%)\end{array}$} & \multirow[t]{2}{*}{$\begin{array}{l}\text { NOS } \\
\text { Score }\end{array}$} \\
\hline & & & & & & & Attempts & Thoughts & \\
\hline $\begin{array}{l}\text { Belik, S. L. et } \\
\text { al (2010)(199) }\end{array}$ & Canada & $\begin{array}{l}\text { Convenience } \\
\text { Sampling } \\
\text { (CS) }\end{array}$ & $\begin{array}{l}\text { The Canadian } \\
\text { Forces }\end{array}$ & $\begin{array}{l}\text { Diagnostic and } \\
\text { Statistical } \\
\text { Manual of } \\
\text { Mental } \\
\text { Disorders-IV } \\
\text { (DSM-IV) }\end{array}$ & - & 37129 & $\begin{array}{l}236(0.8 \\
\%)\end{array}$ & $\begin{array}{l}1613(4.34 \\
\%)\end{array}$ & 8 \\
\hline $\begin{array}{l}\text { Guerra, V. S. et } \\
\text { al }(2011)(120)\end{array}$ & USA & $\begin{array}{l}\text { Convenience } \\
\text { Sampling } \\
\text { (CS) }\end{array}$ & $\begin{array}{l}\text { Veterans in } \\
\text { Operations } \\
\text { Enduring } \\
\text { Freedom and } \\
\text { Iraqi Freedom } \\
\text { (OEF/OIF) }\end{array}$ & $\begin{array}{l}\text { The Beck } \\
\text { Depression } \\
\text { Inventory } \\
\text { (BDI) } \\
\text { Beck Scale for } \\
\text { Suicide Ideation } \\
\text { Scale for Suicide } \\
\text { Ideation-Adapted }\end{array}$ & 38.3 & 393 & $\begin{array}{l}34(8.7 \\
\%)\end{array}$ & $45(11.5 \%)$ & 8 \\
\hline \multirow[t]{6}{*}{$\begin{array}{l}\text { Mansfield, A. } \\
\text { J. et al (2011) } \\
\text { (200) }\end{array}$} & \multirow[t]{6}{*}{ USA } & \multirow{6}{*}{$\begin{array}{l}\text { Convenience } \\
\text { Sampling } \\
\text { (CS) }\end{array}$} & $\begin{array}{l}\text { Military } \\
\text { Personnel }\end{array}$ & \multirow{3}{*}{$\begin{array}{l}\text { The Center for } \\
\text { Epidemiological } \\
\text { Studies } \\
\text { Depression (CES- } \\
\text { D) scale, }\end{array}$} & 28.1 & 3069 & \multirow[t]{6}{*}{ NR } & $215(7 \%)$ & \multirow[t]{6}{*}{6} \\
\hline & & & \multirow{2}{*}{$\begin{array}{l}\text { Military } \\
\text { Personnel (Navy) }\end{array}$} & & 31.8 & 1843 & & $98(5.3 \%)$ & \\
\hline & & & & & \multirow[t]{4}{*}{25.8} & 1226 & & \multirow[t]{4}{*}{$110(9 \%)$} & \\
\hline & & & Military & The PRIME-MD & & & & & \\
\hline & & & $\begin{array}{l}\text { Personnel } \\
\text { (Marine) }\end{array}$ & \multirow{2}{*}{$\begin{array}{l}\text { Patient Health } \\
\text { Questionnaire } \\
(\mathrm{PHQ})\end{array}$} & & & & & \\
\hline & & & & & & $\begin{array}{l}\text { Female } \\
(7255)\end{array}$ & & & \\
\hline $\begin{array}{l}\text { Maguen, S. et } \\
\text { al (2012)(201) }\end{array}$ & USA & $\begin{array}{l}\text { Convenience } \\
\text { Sampling } \\
\text { (CS) }\end{array}$ & Vietnam veterans & Checklist & 40 & 244 & $\begin{array}{l}12(4.9 \\
\%)\end{array}$ & $40(16.4 \%)$ & 6 \\
\hline $\begin{array}{l}\text { Swinkels, C. } \\
\text { M. et al } \\
(2013)(130)\end{array}$ & UK & $\begin{array}{l}\text { Convenience } \\
\text { Sampling } \\
\text { (CS) }\end{array}$ & $\begin{array}{l}\text { U.S. } \\
\text { Afghanistan/Iraq } \\
\text { Veterans }\end{array}$ & $\begin{array}{l}\text { Diagnostic and } \\
\text { Statistical } \\
\text { Manual of } \\
\text { Mental } \\
\text { Disorders-IV } \\
\text { (DSM-IV) }\end{array}$ & 37.40 & 1640 & $\begin{array}{l}132(8 \\
\%)\end{array}$ & NR & 6 \\
\hline $\begin{array}{l}\text { Bryan, C. J. et } \\
\text { al (2013)(202) }\end{array}$ & USA & $\begin{array}{l}\text { Convenience } \\
\text { Sampling } \\
\text { (CS) }\end{array}$ & $\begin{array}{l}\text { Deployed Military } \\
\text { Personnel }\end{array}$ & $\begin{array}{l}\text { The 4-item } \\
\text { Suicidal } \\
\text { Behaviors } \\
\text { Questionnaire- } \\
\text { Revised (SBQ-R) }\end{array}$ & - & 161 & NR & $35(21.7 \%)$ & 5 \\
\hline $\begin{array}{l}\text { Bryan, C. J. et } \\
\text { al (2013)(203) }\end{array}$ & USA & $\begin{array}{l}\text { Convenience } \\
\text { Sampling } \\
\text { (CS) }\end{array}$ & $\begin{array}{l}\text { Deployed Military } \\
\text { Personnel }\end{array}$ & $\begin{array}{l}\text { The 4-item } \\
\text { Suicidal } \\
\text { Behaviors } \\
\text { Questionnaire- } \\
\text { Revised (SBQ-R) }\end{array}$ & - & 158 & $3(1.5 \%)$ & 21 (13.1\%) & 5 \\
\hline $\begin{array}{l}\text { Bryan, C. J. et } \\
\text { al (2013)(204) }\end{array}$ & USA & $\begin{array}{l}\text { Convenience } \\
\text { Sampling } \\
\text { (CS) }\end{array}$ & $\begin{array}{l}\text { Air } \\
\text { Force Personnel }\end{array}$ & $\begin{array}{l}\text { Beck Scale for } \\
\text { Suicidal Ideation- } \\
\text { Current } \\
\text { (BSSI-C) }\end{array}$ & 25.9 & 273 & NR & 53 (19.4\%) & 5 \\
\hline $\begin{array}{l}\text { Bryan, C. J. et } \\
\text { al (2013)(205) }\end{array}$ & USA & $\begin{array}{l}\text { Convenience } \\
\text { Sampling } \\
\text { (CS) }\end{array}$ & $\begin{array}{l}\text { Deployed Military } \\
\text { Personnel }\end{array}$ & $\begin{array}{l}\text { The Self- } \\
\text { Injurious } \\
\text { Thoughts and } \\
\text { Behaviors } \\
\text { Interview (SITBI) }\end{array}$ & 34.2 & 69 & NR & $25(36.2 \%)$ & 5 \\
\hline $\begin{array}{l}\text { Blosnich, J. R. } \\
\text { et al (2014) } \\
(206)\end{array}$ & USA & $\begin{array}{l}\text { Convenience } \\
\text { Sampling } \\
\text { (CS) }\end{array}$ & $\begin{array}{l}\text { Deployed Military } \\
\text { Personnel }\end{array}$ & Checklist & - & 4250 & $N R$ & 154 (3.3\%) & 5 \\
\hline
\end{tabular}




\begin{tabular}{|c|c|c|c|c|c|c|c|c|c|}
\hline \multirow[t]{2}{*}{$\begin{array}{l}\text { Authors } \\
\text { (Years) }\end{array}$} & \multirow[t]{2}{*}{ Country } & \multirow{2}{*}{$\begin{array}{l}\text { Type of } \\
\text { Sampling } \\
\text { (Type of } \\
\text { Study) }\end{array}$} & \multirow[t]{2}{*}{ Study Population } & \multirow{2}{*}{$\begin{array}{l}\text { Depression } \\
\text { Assessment } \\
\text { Method }\end{array}$} & \multirow[t]{2}{*}{$\begin{array}{l}\text { Age } \\
\text { (Mean) }\end{array}$} & \multirow[t]{2}{*}{$\begin{array}{l}\text { Sample } \\
\text { size }\end{array}$} & \multicolumn{2}{|c|}{$\begin{array}{l}\text { Prevalence of Suicide } \\
\text { (\%) }\end{array}$} & \multirow[t]{2}{*}{$\begin{array}{l}\text { NOS } \\
\text { Score }\end{array}$} \\
\hline & & & & & & & Attempts & Thoughts & \\
\hline $\begin{array}{l}\text { Bryan, C. J. et } \\
\text { al (2014)(207) }\end{array}$ & USA & $\begin{array}{l}\text { Convenience } \\
\text { Sampling } \\
\text { (CS) }\end{array}$ & $\begin{array}{l}\text { Deployed Military } \\
\text { Personnel }\end{array}$ & $\begin{array}{l}\text { The Self- } \\
\text { Injurious } \\
\text { Thoughts and } \\
\text { Behaviors } \\
\text { Interview (SITBI) }\end{array}$ & 36.7 & 374 & $\begin{array}{l}29 \\
(7.8 \%)\end{array}$ & $\begin{array}{l}136(36.4 \\
\%)\end{array}$ & 6 \\
\hline $\begin{array}{l}\text { Mash, H. B. et } \\
\text { al (2014)(208) }\end{array}$ & USA & $\begin{array}{l}\text { Convenience } \\
\text { Sampling } \\
\text { (CS) }\end{array}$ & US Army & Checklist & - & 4999 & NR & $303(6 \%)$ & 6 \\
\hline $\begin{array}{l}\text { Don } \\
\text { Richardson, J. } \\
\text { et al (2014) } \\
(135)\end{array}$ & Canada & $\begin{array}{l}\text { Convenience } \\
\text { Sampling } \\
\text { (CS) }\end{array}$ & $\begin{array}{l}\text { Canadian Forces } \\
\text { members and } \\
\text { veterans }\end{array}$ & $\begin{array}{l}\text { The PRIME-MD } \\
\text { Patient Health } \\
\text { Questionnaire } \\
\text { (PHQ) }\end{array}$ & - & 404 & $N R$ & $68(16.8 \%)$ & 7 \\
\hline $\begin{array}{l}\text { Ramsawh, } \mathrm{H}_{\text {. }} \\
\text { J. et al (2014) } \\
\text { (139) }\end{array}$ & USA & $\begin{array}{l}\text { Convenience } \\
\text { Sampling } \\
\text { (CS) }\end{array}$ & $\begin{array}{l}\text { Active Duty } \\
\text { Military } \\
\text { Personnel }\end{array}$ & $\begin{array}{l}\text { 10-item Center } \\
\text { for } \\
\text { Epidemiologic } \\
\text { Studies } \\
\text { Depression Scale }\end{array}$ & 35 & 5461 & $\begin{array}{l}346 \\
(6.33 \%)\end{array}$ & $N R$ & 8 \\
\hline $\begin{array}{l}\text { Bryan, C. J. et } \\
\text { al }(2015)(209)\end{array}$ & USA & $\begin{array}{l}\text { Convenience } \\
\text { Sampling } \\
\text { (CS) }\end{array}$ & $\begin{array}{l}\text { Air Force } \\
\text { personnel }\end{array}$ & $\begin{array}{l}\text { The Suicidal } \\
\text { Behaviors } \\
\text { Questionnaire } \\
\text { Revised (SBQ-R) }\end{array}$ & - & 168 & $2(1.2 \%)$ & $29(17.3 \%)$ & 7 \\
\hline $\begin{array}{l}\text { Cleveland, S. } \\
\text { D. et al (2015) } \\
\text { (141) }\end{array}$ & USA & $\begin{array}{l}\text { Convenience } \\
\text { Sampling } \\
\text { (CS) }\end{array}$ & $\begin{array}{l}\text { Veterans and } \\
\text { Civilian College } \\
\text { Students }\end{array}$ & $\begin{array}{l}\text { Diagnostic and } \\
\text { Statistical } \\
\text { Manual of } \\
\text { Mental } \\
\text { Disorders-IV } \\
\text { (DSM-IV) }\end{array}$ & - & 26969 & $\begin{array}{l}282 \\
(1.07 \%)\end{array}$ & $\begin{array}{l}1730(6.54 \\
\%)\end{array}$ & 8 \\
\hline $\begin{array}{l}\text { Kim, N. Y. et al } \\
\text { (2015) (145) }\end{array}$ & USA & $\begin{array}{l}\text { Convenience } \\
\text { Sampling } \\
\text { (CS) }\end{array}$ & Korean Soldiers & $\begin{array}{l}\text { Scale for suicide } \\
\text { ideation (SSI), } \\
\text { The Beck } \\
\text { Depression } \\
\text { Inventory } \\
\text { (BDI) }\end{array}$ & 21.3 & 414 & NR & $80(19.3 \%)$ & 6 \\
\hline $\begin{array}{l}\text { Ursano, R. J. } \\
\text { et al (2015) } \\
(210)\end{array}$ & USA & $\begin{array}{l}\text { Convenience } \\
\text { Sampling } \\
\text { (CS) }\end{array}$ & Soldiers & $\begin{array}{l}\text { The Columbia } \\
\text { Suicidal Severity } \\
\text { Rating Scale (C- } \\
\text { SSRS) }\end{array}$ & 20 & 38237 & $\begin{array}{l}536(1.9 \\
\%)\end{array}$ & $\begin{array}{l}5353(14 \\
\%)\end{array}$ & 8 \\
\hline $\begin{array}{l}\text { Vanderploeg, } \\
\text { R. D. et al } \\
\text { (2015) (150) }\end{array}$ & USA & $\begin{array}{l}\text { Convenience } \\
\text { Sampling } \\
\text { (CS) }\end{array}$ & $\begin{array}{l}\text { Florida National } \\
\text { Guard } \\
\text { Members }\end{array}$ & $\begin{array}{l}\text { The PRIME-MD } \\
\text { Patient Health } \\
\text { Questionnaire } \\
\text { (PHQ) }\end{array}$ & - & 3098 & NR & $130(4.2 \%)$ & 7 \\
\hline $\begin{array}{l}\text { Forbes, D. et } \\
\text { al }(2016)(152)\end{array}$ & Australia & $\begin{array}{l}\text { Convenience } \\
\text { Sampling } \\
\text { (CS) }\end{array}$ & $\begin{array}{l}\text { Australian } \\
\text { peacekeepers }\end{array}$ & $\begin{array}{l}\text { Diagnostic and } \\
\text { Statistical } \\
\text { Manual of } \\
\text { Mental } \\
\text { Disorders-IV } \\
\text { (DSM-IV) }\end{array}$ & 46.5 & 2050 & $\begin{array}{l}25(1.2 \\
\%)\end{array}$ & $\begin{array}{l}275(13.4 \\
\%)\end{array}$ & 8 \\
\hline Herberman & USA & $\begin{array}{l}\text { Convenience } \\
\text { Sampling }\end{array}$ & $\begin{array}{l}\text { U.S. Army } \\
\text { soldiers }\end{array}$ & The 10-item & - & 3813 & $\begin{array}{l}230(6 \\
\%)\end{array}$ & NR & 8 \\
\hline & & (CS) & $\begin{array}{l}\text { U.S. Army } \\
\text { soldiers with } \\
\text { alcohol use }\end{array}$ & $\begin{array}{l}\text { Epidemiologic } \\
\text { Studies } \\
\text { Depression Scale }\end{array}$ & & 1210 & $\begin{array}{l}100(8.3 \\
\%)\end{array}$ & & \\
\hline
\end{tabular}




\begin{tabular}{|c|c|c|c|c|c|c|c|c|c|}
\hline \multirow[t]{2}{*}{$\begin{array}{l}\text { Authors } \\
\text { (Years) }\end{array}$} & \multirow[t]{2}{*}{ Country } & \multirow{2}{*}{$\begin{array}{l}\text { Type of } \\
\text { Sampling } \\
\text { (Type of } \\
\text { Study) }\end{array}$} & \multirow[t]{2}{*}{ Study Population } & \multirow{2}{*}{$\begin{array}{l}\text { Depression } \\
\text { Assessment } \\
\text { Method }\end{array}$} & \multirow[t]{2}{*}{$\begin{array}{l}\text { Age } \\
\text { (Mean) }\end{array}$} & \multirow[t]{2}{*}{$\begin{array}{l}\text { Sample } \\
\text { size }\end{array}$} & \multicolumn{2}{|c|}{$\begin{array}{l}\text { Prevalence of Suicide } \\
\text { (\%) }\end{array}$} & \multirow[t]{2}{*}{$\begin{array}{l}\text { NOS } \\
\text { Score }\end{array}$} \\
\hline & & & & & & & Attempts & Thoughts & \\
\hline \multirow[t]{3}{*}{$\begin{array}{l}\text { Monteith, L. L. } \\
\text { et al (2016) } \\
(156)\end{array}$} & \multirow[t]{3}{*}{ USA } & \multirow{3}{*}{$\begin{array}{l}\text { Convenience } \\
\text { Sampling } \\
\text { (CS) }\end{array}$} & \multirow[t]{3}{*}{ Veterans } & \multirow{3}{*}{$\begin{array}{l}\text { Beck Scale for } \\
\text { Suicide Ideation } \\
\text { (BSS), } \\
\text { Multidimensional } \\
\text { Suicide } \\
\text { Inventory-28 } \\
\text { (MSI) Negative } \\
\text { Affect scale }\end{array}$} & \multirow[t]{3}{*}{49.6} & $\begin{array}{l}\text { Total } \\
(354)\end{array}$ & $\begin{array}{l}92(26.8 \\
\%)\end{array}$ & \multirow[t]{3}{*}{ NR } & \multirow[t]{3}{*}{8} \\
\hline & & & & & & $\begin{array}{l}\text { Male } \\
(310)\end{array}$ & $\begin{array}{l}82(26.5 \\
\%)\end{array}$ & & \\
\hline & & & & & & $\begin{array}{l}\text { Female } \\
(44)\end{array}$ & $\begin{array}{l}13(29.5 \\
\%)\end{array}$ & & \\
\hline \multirow{4}{*}{$\begin{array}{l}\text { Cohen, G. H. } \\
\text { et al (2017) } \\
(160)\end{array}$} & \multirow[t]{4}{*}{ USA } & \multirow{4}{*}{$\begin{array}{l}\text { Convenience } \\
\text { Sampling } \\
\text { (CS) }\end{array}$} & \multirow{2}{*}{$\begin{array}{l}\text { Army National } \\
\text { Guard Soldiers }\end{array}$} & \multirow{4}{*}{$\begin{array}{l}\text { The PRIME-MD } \\
\text { Patient Health } \\
\text { Questionnaire } \\
\text { (PHQ), } \\
\text { The PHQ-9 Item }\end{array}$} & \multirow[t]{4}{*}{ - } & 1582 & \multirow[t]{4}{*}{ NR } & $42(2.6 \%)$ & \multirow[t]{4}{*}{8} \\
\hline & & & & & & & & & \\
\hline & & & \multirow[t]{2}{*}{$\begin{array}{l}\text { Army National } \\
\text { with Alcohol Use }\end{array}$} & & & 93 & & $8(8.6 \%)$ & \\
\hline & & & & & & & & & \\
\hline \multirow{6}{*}{$\begin{array}{l}\text { Gradus, J. L. } \\
\text { et al (2017) } \\
\text { (161) }\end{array}$} & \multirow[t]{6}{*}{ USA } & \multirow{6}{*}{$\begin{array}{l}\text { Random } \\
\text { Sampling } \\
\text { (CS) }\end{array}$} & \multirow{6}{*}{$\begin{array}{l}\text { Veterans of the } \\
\text { Iraq and } \\
\text { Afghanistan } \\
\text { Wars }\end{array}$} & \multirow{3}{*}{$\begin{array}{l}20- \\
\text { item, self-report } \\
\text { Center for } \\
\text { Epidemiological } \\
\text { Studies } \\
\text { Depression Scale } \\
\text { (CES-D), }\end{array}$} & \multirow[t]{6}{*}{34} & $\begin{array}{l}\text { Total } \\
(2244)\end{array}$ & NR & $\begin{array}{l}370(16.5 \\
\%)\end{array}$ & 7 \\
\hline & & & & & & & & & \\
\hline & & & & & & $\begin{array}{l}\text { Male } \\
(1062)\end{array}$ & & $\begin{array}{l}179 \\
(16.9 \%)\end{array}$ & \\
\hline & & & & The 4-item & & & & & \\
\hline & & & & Behaviors & & $\begin{array}{l}\text { Female } \\
\text { (1099) }\end{array}$ & & $\begin{array}{l}191 \\
(17.4 \%)\end{array}$ & \\
\hline & & & & $\begin{array}{l}\text { Questionnaire- } \\
\text { Short Form (SBQ- } \\
\text { SF) }\end{array}$ & & & & & \\
\hline $\begin{array}{l}\text { Weeks, M. et } \\
\text { al (2017)(163) }\end{array}$ & Canada & $\begin{array}{l}\text { Convenience } \\
\text { Sampling } \\
\text { (CS) }\end{array}$ & $\begin{array}{l}\text { Canadian Military } \\
\text { and Civilian } \\
\text { Populations }\end{array}$ & $\begin{array}{l}\text { Diagnostic and } \\
\text { Statistical } \\
\text { Manual of } \\
\text { Mental } \\
\text { Disorders-IV }\end{array}$ & 35 & 6696 & NR & $289(4 . \%)$ & 8 \\
\hline & & & & (DSM-IV) & & & & & \\
\hline $\begin{array}{l}\text { Bartlett, B. A. } \\
\text { et al (2018) } \\
(164)\end{array}$ & USA & $\begin{array}{l}\text { Convenience } \\
\text { Sampling }\end{array}$ & Military veterans & $20-$ & 38.40 & 910 & $\begin{array}{l}62(7.5 \\
\%)\end{array}$ & NR & 6 \\
\hline & & (CS) & & $\begin{array}{l}\text { Center for } \\
\text { Epidemiological } \\
\text { Studies } \\
\text { Depression Scale } \\
\text { (CES-D) }\end{array}$ & & & & & \\
\hline $\begin{array}{l}\text { Boulos, D. et } \\
\text { al (2018)(166) }\end{array}$ & Canada & $\begin{array}{l}\text { Random } \\
\text { Sampling }\end{array}$ & $\begin{array}{l}\text { Regular Force } \\
\text { personnel }\end{array}$ & $\begin{array}{l}\text { Diagnostic and } \\
\text { Statistical }\end{array}$ & - & 3385 & NR & $156(4.6 \%)$ & 7 \\
\hline & & & $\begin{array}{l}\text { Reserve Force } \\
\text { personnel }\end{array}$ & Disorders-IV & & 1469 & & $82(5.6 \%)$ & \\
\hline & & & & (DSM-IV) & & & & & \\
\hline $\begin{array}{l}\text { Dillon, K. H. et } \\
\text { al }(2018)(167)\end{array}$ & USA & $\begin{array}{l}\text { Convenience } \\
\text { Sampling }\end{array}$ & $\begin{array}{l}\text { Iraq/Afghanistan- } \\
\text { era veterans }\end{array}$ & $\begin{array}{l}\text { The Beck Scale } \\
\text { for Suicide }\end{array}$ & - & 3238 & $\begin{array}{l}291(9 \\
\%)\end{array}$ & NR & 7 \\
\hline & & (CS) & & $\begin{array}{l}\text { The Structured } \\
\text { Clinical Interview } \\
\text { for DSM-IV-TR } \\
\text { (SCID) }\end{array}$ & & & & & \\
\hline
\end{tabular}




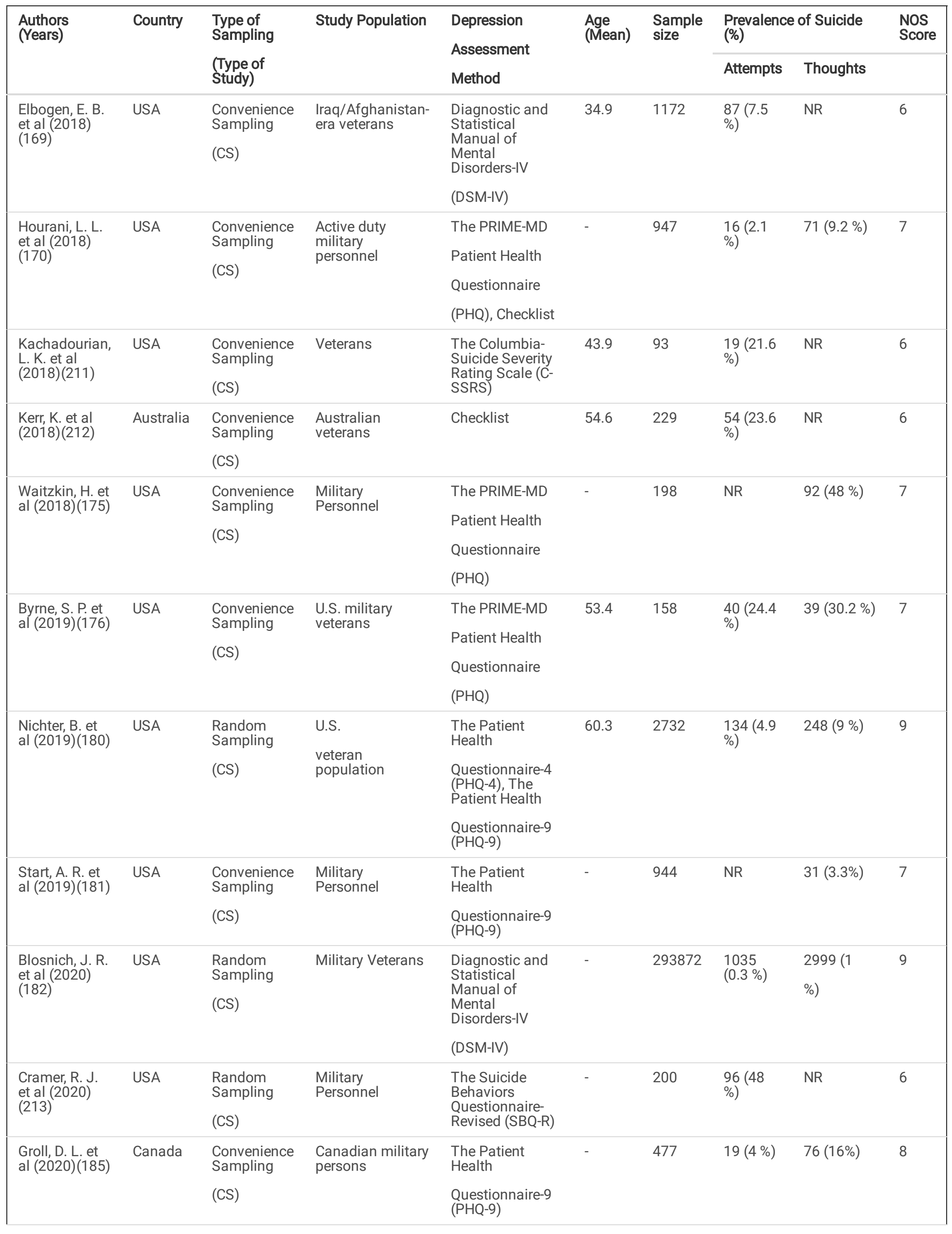




\begin{tabular}{|c|c|c|c|c|c|c|c|c|c|}
\hline \multirow[t]{2}{*}{$\begin{array}{l}\text { Authors } \\
\text { (Years) }\end{array}$} & \multirow[t]{2}{*}{ Country } & \multirow{2}{*}{$\begin{array}{l}\text { Type of } \\
\text { Sampling } \\
\text { (Type of } \\
\text { Study) }\end{array}$} & \multirow[t]{2}{*}{ Study Population } & \multirow{2}{*}{$\begin{array}{l}\text { Depression } \\
\text { Assessment } \\
\text { Method }\end{array}$} & \multirow[t]{2}{*}{$\begin{array}{l}\text { Age } \\
\text { (Mean) }\end{array}$} & \multirow[t]{2}{*}{$\begin{array}{l}\text { Sample } \\
\text { size }\end{array}$} & \multicolumn{2}{|c|}{$\begin{array}{l}\text { Prevalence of Suicide } \\
(\%)\end{array}$} & \multirow[t]{2}{*}{$\begin{array}{l}\text { NOS } \\
\text { Score }\end{array}$} \\
\hline & & & & & & & Attempts & Thoughts & \\
\hline \multirow[t]{3}{*}{$\begin{array}{l}\text { Shim, E. J. et } \\
\text { al (2020)(187) }\end{array}$} & \multirow[t]{3}{*}{ Korea } & \multirow{3}{*}{$\begin{array}{l}\text { Random } \\
\text { Sampling } \\
\text { (CS) }\end{array}$} & \multirow[t]{3}{*}{$\begin{array}{l}\text { Korean military } \\
\text { population }\end{array}$} & The Mini & \multirow[t]{3}{*}{50.6} & \multirow[t]{3}{*}{1937} & \multirow[t]{3}{*}{$\begin{array}{l}87(4.5 \\
\%)\end{array}$} & \multirow[t]{3}{*}{ NR } & \multirow[t]{3}{*}{8} \\
\hline & & & & $\begin{array}{l}\text { International } \\
\text { Neuropsychiatric } \\
\text { Interview Plus } \\
\text { (MINI-Plus), The } \\
\text { Patient Health }\end{array}$ & & & & & \\
\hline & & & & $\begin{array}{l}\text { Questionnaire-9 } \\
\text { (PHQ-9) }\end{array}$ & & & & & \\
\hline \multirow{3}{*}{$\begin{array}{l}\text { Smigelsky, M. } \\
\text { A. et al (2020) } \\
\text { (188) }\end{array}$} & \multirow[t]{3}{*}{ USA } & $\begin{array}{l}\text { Convenience } \\
\text { Sampling }\end{array}$ & \multirow[t]{3}{*}{$\begin{array}{l}\text { U.S. military } \\
\text { population }\end{array}$} & $\begin{array}{l}\text { Diagnostic and } \\
\text { Statistical }\end{array}$ & \multirow[t]{3}{*}{37.6} & \multirow[t]{3}{*}{1002} & \multirow[t]{3}{*}{$41(4 \%)$} & \multirow[t]{3}{*}{ NR } & \multirow[t]{3}{*}{6} \\
\hline & & (CS) & & $\begin{array}{l}\text { Mental } \\
\text { Disorders-IV }\end{array}$ & & & & & \\
\hline & & & & (DSM-IV) & & & & & \\
\hline \multirow{3}{*}{$\begin{array}{l}\text { Stefanovics, } \\
\text { E. A. et al al } \\
(2020)(190)\end{array}$} & \multirow[t]{3}{*}{ USA } & $\begin{array}{l}\text { Convenience } \\
\text { Sampling }\end{array}$ & \multirow[t]{3}{*}{$\begin{array}{l}\text { U.S. Military } \\
\text { Veterans }\end{array}$} & $\begin{array}{l}\text { The Mini } \\
\text { International }\end{array}$ & \multirow[t]{3}{*}{55} & \multirow[t]{3}{*}{1308} & \multirow[t]{3}{*}{$\begin{array}{l}118(9 \\
\%)\end{array}$} & \multirow[t]{3}{*}{$\begin{array}{l}165(12.6 \\
\%)\end{array}$} & 5 \\
\hline & & (CS) & & $\begin{array}{l}\text { Interview (MINI), } \\
\text { The Patient } \\
\text { Health }\end{array}$ & & & & & \\
\hline & & & & $\begin{array}{l}\text { Questionnaire-9 } \\
\text { (PHQ-9) }\end{array}$ & & & & & \\
\hline $\begin{array}{l}\text { Wang, J. et al } \\
(2020)(192)\end{array}$ & USA & $\begin{array}{l}\text { Convenience } \\
\text { Sampling }\end{array}$ & U.S. & $\begin{array}{l}\text { The Patient } \\
\text { Health }\end{array}$ & 34.4 & 3503 & NR & $101(2.9 \%)$ & 6 \\
\hline & & (CS) & $\begin{array}{l}\text { Reserve and } \\
\text { National Guard } \\
\text { Personnel }\end{array}$ & $\begin{array}{l}\text { Questionnaire-9 } \\
\text { (PHQ-9) }\end{array}$ & & & & & \\
\hline $\begin{array}{l}\text { Anestis, M. D. } \\
\text { et al (2020) } \\
(214)\end{array}$ & USA & $\begin{array}{l}\text { Convenience } \\
\text { Sampling } \\
\text { (CS) }\end{array}$ & $\begin{array}{l}\text { U.S. Military } \\
\text { Veterans }\end{array}$ & $\begin{array}{l}\text { The Suicide } \\
\text { Behaviors } \\
\text { Questionnaire- } \\
\text { Revised (SBQ-R) }\end{array}$ & 27.0 & 953 & NR & $\begin{array}{l}105 \\
(15.2 \%)\end{array}$ & 5 \\
\hline $\begin{array}{l}\text { Monteith, L. L. } \\
\text { et al }(2020)\end{array}$ & USA & $\begin{array}{l}\text { Convenience } \\
\text { Sampling }\end{array}$ & Female veterans & Checklist & 55.6 & 439 & $\begin{array}{l}158(36 \\
\%)\end{array}$ & $113(25.7 \%)$ & 5 \\
\hline & & (CS) & & & & & & & \\
\hline $\begin{array}{l}\text { Ursano, R. J. } \\
\text { et al (2020) } \\
(193)\end{array}$ & USA & $\begin{array}{l}\text { Convenience } \\
\text { Sampling }\end{array}$ & $\begin{array}{l}\text { US Army Soldiers } \\
\text { During } \\
\text { Deployment }\end{array}$ & $\begin{array}{l}\text { Diagnostic and } \\
\text { Statistical } \\
\text { Manual of }\end{array}$ & - & 3957 & NR & $85(2.1 \%)$ & 7 \\
\hline & & & in Afghanistan & Disorders-IV & & & & & \\
\hline & & & & (DSM-IV) & & & & & \\
\hline $\begin{array}{l}\text { Yeom, C. W. et } \\
\text { al (2020)(194) }\end{array}$ & Korea & $\begin{array}{l}\text { Convenience } \\
\text { Sampling }\end{array}$ & $\begin{array}{l}\text { Korean military } \\
\text { personal }\end{array}$ & The Mini & 21.4 & 480 & $\begin{array}{l}22(4.5 \\
\%)\end{array}$ & NR & 6 \\
\hline & & (CS) & & $\begin{array}{l}\text { International } \\
\text { Neuropsychiatric } \\
\text { Interview Plus }\end{array}$ & & & & & \\
\hline & & & & $\begin{array}{l}\text { (MINI-Plus } \\
\text { Suicidality } \\
\text { module), The } \\
\text { Patient Health }\end{array}$ & & & & & \\
\hline & & & & $\begin{array}{l}\text { Questionnaire-9 } \\
\text { (PHQ-9) }\end{array}$ & & & & & \\
\hline
\end{tabular}

\section{Quantitative analysis}

\section{Prevalence of depression in the all military}

Initially, the studies were divided into two groups: the active duty military community and the veteran's community in terms of the study population. Then, separate analyzes were performed for each of these communities and the prevalence of depression in each was meta-analyzed. Of the 133 final selected cross-sectional studies, 80 were in the veterans and 100 were in the active duty military personnel. 


\section{Prevalence of depression in the active duty military}

In these studies, 1278837 employees of the active or serving military had been examined, of whom 273173 had depression. After combining the results of these studies, the overall pooled prevalence of depression in the active or in-service military was $23 \%$ with a confidence interval of 20 to $26 \%$. The percentage of heterogeneity was $99.91 \%$ which was statistically significant (Table 3 ). 
Table 3

The pooled estimate of prevalence of depression in active duty and veteran military

\begin{tabular}{|c|c|c|c|c|c|c|c|}
\hline \multirow[t]{2}{*}{ Categories } & \multirow[t]{2}{*}{$\begin{array}{l}\text { No. of Studies (Sample } \\
\text { Size) }\end{array}$} & \multirow[t]{2}{*}{$\begin{array}{l}\text { Pooled Prevalence (\% } 95 \\
\mathrm{Cl})\end{array}$} & \multicolumn{3}{|c|}{$\begin{array}{l}\text { Between studies heterogeneity } \\
\text { assessment (\%) }\end{array}$} & \multicolumn{2}{|c|}{$\begin{array}{l}\text { Between subgroups } \\
\text { heterogeneity } \\
\text { assessment (\%) }\end{array}$} \\
\hline & & & $R$ & $P_{\text {Heterogenity }}$ & $Z$ & Q & $P_{\text {Heterogenity }}$ \\
\hline \multicolumn{8}{|c|}{ The prevalence of depression in active duty military } \\
\hline Total & $100(1278837)$ & $23 \%(20-26 \%)$ & $\begin{array}{l}87.91 \\
\%\end{array}$ & 0.018 & 27.74 & - & - \\
\hline Sampling Method & 67 (939796) & $21 \%(18-25 \%)$ & \multirow{2}{*}{$\begin{array}{l}66.90 \\
\% \\
54.80 \\
\%\end{array}$} & 0.030 & 20.25 & \multirow[t]{2}{*}{9.33} & \multirow[t]{2}{*}{0.001} \\
\hline $\begin{array}{l}\text { Convinces Sampling } \\
\text { Random Sampling }\end{array}$ & $33(339041)$ & 26 \% (19-32 \%) & & 0.050 & 13.11 & & \\
\hline Type of Forces & $5(4562)$ & $20 \%(9-33 \%)$ & \multirow{3}{*}{$\begin{array}{l}83.93 \\
\% \\
89.45 \\
\%\end{array}$} & 0.040 & 5.59 & \multirow[t]{5}{*}{8.98} & \multirow[t]{5}{*}{0.001} \\
\hline Air Forces & $36(995073)$ & $22 \%(20-23 \%)$ & & 0.034 & 20.05 & & \\
\hline Armed Forces & 6 (775778) & $31 \%(16-48 \%)$ & & 0.0001 & 6.22 & & \\
\hline Marine Forces & $53(201624)$ & $22 \%(16-28 \%)$ & $\begin{array}{l}90.86 \\
\%\end{array}$ & 0.005 & 12.41 & & \\
\hline Military Forces & & & $\begin{array}{l}79.89 \\
\%\end{array}$ & & & & \\
\hline Population & $90(1152451)$ & $22 \%(20-25 \%)$ & \multirow{2}{*}{$\begin{array}{l}99.87 \\
\%\end{array}$} & 0.0001 & 18.28 & \multirow[t]{5}{*}{10.03} & \multirow[t]{5}{*}{0.001} \\
\hline Healthy Forces & $3(113620)$ & $15 \%(3-36 \%)$ & & - & 3.16 & & \\
\hline Forces with HIV/AIDS & $5(8303)$ & $29 \%(13-47 \%)$ & \multirow{3}{*}{$\begin{array}{l}99.96 \\
\% \\
-\end{array}$} & 0.0001 & 5.70 & & \\
\hline Forces with Alcohol Use & $2(4463)$ & $37 \%(36-39 \%)$ & & - & 18.04 & & \\
\hline $\begin{array}{l}\text { Forces with Substance } \\
\text { Use }\end{array}$ & & & & & & & \\
\hline Gender & $71(1163273)$ & $22 \%(20-25 \%)$ & \multirow{3}{*}{$\begin{array}{l}90.88 \\
\% \\
91.83 \\
\%\end{array}$} & 0.0001 & 20.19 & \multirow[t]{4}{*}{10.01} & \multirow[t]{4}{*}{0.001} \\
\hline Total & 20 (110847) & $23 \%(12-37 \%)$ & & 0.0001 & 6.45 & & \\
\hline Male & $9(4717)$ & $25 \%(13-40 \%)$ & & 0.012 & 6.15 & & \\
\hline Female & & & \multicolumn{3}{|l|}{$\begin{array}{l}89.99 \\
\%\end{array}$} & & \\
\hline Tools & $9(38888)$ & $25 \%(15-36 \%)$ & 65.75 & 0.054 & 5.88 & \multirow[t]{11}{*}{5.09} & \multirow[t]{11}{*}{0.001} \\
\hline BDI Scale & $7(15365)$ & $13 \%(8-19 \%)$ & \multirow{2}{*}{$\begin{array}{l}50.20 \\
\%\end{array}$} & 0.130 & 4.05 & & \\
\hline CES-D Scale & $13(16980)$ & $25 \%(17-35 \%)$ & & 0.060 & 10.22 & & \\
\hline Interviews & $36(202430)$ & $15 \%(11-19 \%)$ & 67.38 & 0.078 & 12.41 & & \\
\hline DSM-IV Scale & $1(236)$ & $56 \%(49-60 \%)$ & \multirow{2}{*}{$\begin{array}{l}60.07 \\
\%\end{array}$} & - & - & & \\
\hline BSI Scale & 1 (197) & $47 \%(40-54 \%)$ & & - & - & & \\
\hline HAMD Scale & $1(6943)$ & $10 \%(9-11 \%)$ & - & - & - & & \\
\hline HADS Scale & 24 (692087) & $15 \%(13-17 \%)$ & - & 0.059 & 9.32 & & \\
\hline PHQ Scale & $5(304767)$ & $20 \%(14-26 \%)$ & - & 0.059 & 9.03 & & \\
\hline \multirow[t]{2}{*}{ SDS Scale } & & & \multicolumn{3}{|l|}{$\begin{array}{l}78.62 \\
\%\end{array}$} & & \\
\hline & & & \multicolumn{2}{|l|}{$\begin{array}{l}72.99 \\
\%\end{array}$} & & & \\
\hline
\end{tabular}

Beck Depression Inventory (BDI), Center for Epidemiological Studies Depression (CES-D), Clinical Symptoms (Interviews), Diagnostic and Statistical Manual of Mental Disorders-IV (DSM-IV), The Brief Symptom Inventory (BSI), The Hamilton Depression Rating Scale (HAMD), The Hospital Anxiety and Depression Scale (HADS), The Patient Health Questionnaire(PHQ), The Zung Self-Report Depression Scale (SDS), Geriatric Depression Scale (GDS), Mental Health Inventory (MHI), Quick Inventory of Depressive Symptomatology (QIDS), Hamilton Depression Rating Scale (HDRS)-24 item, 


\begin{tabular}{|c|c|c|c|c|c|c|c|}
\hline \multirow[t]{2}{*}{ Categories } & \multirow[t]{2}{*}{$\begin{array}{l}\text { No. of Studies (Sample } \\
\text { Size) }\end{array}$} & \multirow[t]{2}{*}{$\begin{array}{l}\text { Pooled Prevalence (\% } 95 \\
\mathrm{Cl})\end{array}$} & \multicolumn{3}{|c|}{$\begin{array}{l}\text { Between studies heterogeneity } \\
\text { assessment (\%) }\end{array}$} & \multicolumn{2}{|c|}{$\begin{array}{l}\text { Between subgroups } \\
\text { heterogeneity } \\
\text { assessment (\%) }\end{array}$} \\
\hline & & & $R$ & $P_{\text {Heterogenity }}$ & $Z$ & Q & $P_{\text {Heterogenity }}$ \\
\hline \multicolumn{8}{|c|}{ The prevalence of depression in veteran military } \\
\hline Total & $80(887982)$ & $20 \%(18-22 \%)$ & $\begin{array}{l}79.80 \\
\%\end{array}$ & 0.032 & 31.46 & - & - \\
\hline Sampling Method & $55(565979)$ & $19 \%(16-21 \%)$ & \multirow{2}{*}{$\begin{array}{l}69.78 \\
\% \\
58.26 \\
\%\end{array}$} & 0.049 & 17.25 & \multirow[t]{2}{*}{2.12} & \multirow[t]{2}{*}{0.150} \\
\hline $\begin{array}{l}\text { Convinces Sampling } \\
\text { Random Sampling }\end{array}$ & $25(322003)$ & $22 \%(18-27 \%)$ & & 0.054 & 10.02 & & \\
\hline Type of Forces & NR & - & - & - & - & \multirow[t]{4}{*}{1.27} & \multirow[t]{4}{*}{0.260} \\
\hline Air Forces & $68(583048)$ & $19 \%(17-22 \%)$ & \multirow{2}{*}{$\begin{array}{l}76.97 \\
\%\end{array}$} & 0.054 & 16.88 & & \\
\hline Armed Forces & NR & - & & - & - & & \\
\hline $\begin{array}{l}\text { Marine Forces } \\
\text { Military Forces }\end{array}$ & $12(304934)$ & $24 \%(16-33 \%)$ & $\begin{array}{l}64.66 \\
\%\end{array}$ & 0.034 & 9.83 & & \\
\hline Population & $64(856091)$ & $19 \%(17-22 \%)$ & \multirow{3}{*}{$\begin{array}{l}99.09 \\
\% \\
91.33 \\
\%\end{array}$} & 0.0001 & 18.28 & \multirow[t]{6}{*}{28.40} & \multirow[t]{6}{*}{0.001} \\
\hline Healthy Forces & $2(1257)$ & $16 \%(14-18 \%)$ & & 0.0001 & 22.32 & & \\
\hline Forces with HIV/AIDS & $4(1780)$ & $29 \%(21-37 \%)$ & & 0.0001 & 11.92 & & \\
\hline Forces with Alcohol Use & 4 (4397) & $10 \%(6-14 \%)$ & \multirow{3}{*}{$\begin{array}{l}98.44 \\
\% \\
74.50 \\
\% \\
88.93\end{array}$} & 0.0001 & 8.68 & & \\
\hline $\begin{array}{l}\text { Forces with Substance } \\
\text { Use }\end{array}$ & $6(24457)$ & $29 \%(17-43 \%)$ & & 0.001 & 7.36 & & \\
\hline Forces with $\mathrm{HCV}$ & & & & & & & \\
\hline Gender & $55(237654)$ & $20 \%(17-23 \%)$ & \multirow{3}{*}{$\begin{array}{l}90.88 \\
\% \\
91.91 \\
\%\end{array}$} & 0.0001 & 22.36 & \multirow[t]{4}{*}{0.12} & \multirow[t]{4}{*}{0.873} \\
\hline Total & $15(343584)$ & $21 \%(13-31 \%)$ & & 0.0001 & 7.75 & & \\
\hline Male & $10(306744)$ & $20 \%(14-26 \%)$ & & 0.0001 & 11.40 & & \\
\hline Female & & & $\begin{array}{l}88.49 \\
\%\end{array}$ & & & & \\
\hline
\end{tabular}

Beck Depression Inventory (BDI), Center for Epidemiological Studies Depression (CES-D), Clinical Symptoms (Interviews), Diagnostic and Statistical Manual of Mental Disorders-IV (DSM-IV), The Brief Symptom Inventory (BSI), The Hamilton Depression Rating Scale (HAMD), The Hospital Anxiety and Depression Scale (HADS), The Patient Health Questionnaire(PHQ), The Zung Self-Report Depression Scale (SDS), Geriatric Depression Scale (GDS), Mental Health Inventory (MHI), Quick Inventory of Depressive Symptomatology (QIDS), Hamilton Depression Rating Scale (HDRS)-24 item, 


\begin{tabular}{|c|c|c|c|c|c|c|c|}
\hline \multirow[t]{2}{*}{ Categories } & \multirow[t]{2}{*}{$\begin{array}{l}\text { No. of Studies (Sample } \\
\text { Size) }\end{array}$} & \multirow[t]{2}{*}{$\begin{array}{l}\text { Pooled Prevalence (\% } 95 \\
\text { Cl) }\end{array}$} & \multicolumn{3}{|c|}{$\begin{array}{l}\text { Between studies heterogeneity } \\
\text { assessment (\%) }\end{array}$} & \multicolumn{2}{|c|}{$\begin{array}{l}\text { Between subgroups } \\
\text { heterogeneity } \\
\text { assessment (\%) }\end{array}$} \\
\hline & & & $R$ & $P_{\text {Heterogenity }}$ & $Z$ & Q & $P_{\text {Heterogenity }}$ \\
\hline Tools & 7 (415692) & $14 \%(9-21 \%)$ & \multirow{2}{*}{$\begin{array}{l}55.15 \\
\%\end{array}$} & 0.060 & 7.97 & \multirow[t]{13}{*}{22.16} & \multirow[t]{13}{*}{0.001} \\
\hline BDI Scale & $11(318802)$ & $18 \%(13-25 \%)$ & & 0.761 & 10.80 & & \\
\hline CES-D Scale & $13(50675)$ & $20 \%(11-31 \%)$ & $\begin{array}{l}40.45 \\
\%\end{array}$ & 0.181 & 6.74 & & \\
\hline Interviews & 11 (64263) & $15 \%(9-22 \%)$ & \multirow{3}{*}{$\begin{array}{l}60.22 \\
\% \\
78.99 \\
\%\end{array}$} & 0.028 & 7.54 & & \\
\hline DSM-IV Scale & $29(28445)$ & $21 \%(17-25 \%)$ & & 0.049 & 17.52 & & \\
\hline PHQ Scale & $2(1300)$ & $47 \%(44-50 \%)$ & & 0.059 & 9.14 & & \\
\hline SDS Scale & 1 (1032) & $37 \%(34-40 \%)$ & \multirow{3}{*}{$\begin{array}{l}\begin{array}{l}78.48 \\
\% \\
52.04 \\
\%\end{array} \\
\end{array}$} & - & 20.91 & & \\
\hline GDS Scale & $4(3649)$ & $35 \%(15-59 \%)$ & & 0.601 & 4.91 & & \\
\hline MHI Scale & 1 (1002) & $21 \%(18-24 \%)$ & & - & 19.13 & & \\
\hline QIDS Scale & 1 (3122) & $7 \%(6-8 \%)$ & - & - & 18.28 & & \\
\hline \multirow[t]{3}{*}{ HDRS Scale } & & & \multicolumn{3}{|l|}{$\begin{array}{l}50.74 \\
\%\end{array}$} & & \\
\hline & & & \multicolumn{3}{|l|}{-} & & \\
\hline & & & \multicolumn{3}{|l|}{ - } & & \\
\hline \multicolumn{8}{|c|}{$\begin{array}{l}\text { Beck Depression Inventory (BDI), Center for Epidemiological Studies Depression (CES-D), Clinical Symptoms (Interviews), Diagnostic and } \\
\text { Statistical Manual of Mental Disorders-IV ( (SSM-IV), The Brief Symptom Inventory (BSI)), The Hamilton Depression Rating Scale (HAMD), The } \\
\text { Hospital Anxiety and Depression Scale (HADS), The Patient Health Questionnaire(PHQ), The Zung Self-Report Depression Scale (SDS), Geriatric } \\
\text { Depression Scale (GDS), Mental Health Inventory (MHI), Quick Inventory of Depressive Symptomatology (QIDS), Hamilton Depression Rating } \\
\text { Scale (HDRS)-24 item, }\end{array}$} \\
\hline
\end{tabular}

The pooled prevalence of depression was $21 \%$ (\% $95 \mathrm{Cl} ; 18-25 \%$ ) in studies where the sampling method was the available one (convinces sampling). A total of 67 studies used this type of sampling method, which had examined a total of 939,796 active members, of whom 21,7487 had been considered depressed. In addition, 33 studies with a sample size of 339041 people had used the random sampling method to collect their samples. After combining these studies, the pooled prevalence of depression was estimated to be $26 \%(\% 95 \mathrm{Cl} ; 19-32 \%$ ) (Table 3).

In this meta-analysis, the pooled prevalence of depression in active duty military personnel was also calculated based on the location and the results were reported in Table 3. The results showed that the pooled prevalence of depression in active air, land, and naval forces was $20 \%$ (\% $95 \mathrm{Cl}$; 9-33\%), 22\% (\% $95 \mathrm{Cl} ; 20-23 \%$ ), and 31\% (\% $95 \mathrm{Cl} ; 16-48 \%$ ), respectively. In 53 cross-sectional studies, it had not been specified that in which military unit, the study population was serving and it had been mentioned as military forces in that studies, so, a group called military forces was formed, the sample size of which was equal to 201624 active military personnel of whom 65,158 people were depressed. The pooled prevalence of depression after a combination of these studies was $22 \%$ (\% $95 \mathrm{Cl} ; 16-28 \%$ ) (Table 3 ).

The pooled prevalence of depression in active militaries with HIV was $15 \%(\% 95 \mathrm{Cl} ; 3-36 \%$ ), in active militaries with substance use was $37 \%$ (\% 95 $\mathrm{Cl} ; 36-39 \%)$, in militaries using alcohol was equal to $29 \%(\% 95 \mathrm{Cl} ; 13-47 \%)$ and finally in healthy and disease-free military members was equal to $22 \%$ (\% $95 \mathrm{Cl} ; 20-25 \%$ ) (Table 3).

The pooled prevalence of depression in the active military varied by gender. A total of 71 cross-sectional studies had not identified the gender of the study population while 20 and 9 studies had been performed on military men and women, respectively. In studies that had not specified gender, the sample size was 1163273 people, of whom 221910 individuals were depressed. The sample size in cross-sectional studies on military men and women was 110847 and 4717 people, respectively, of whom 50370 and 893 were depressed, respectively. The results of meta-analysis showed that the pooled prevalence of depression in male soldiers was equal to $23 \%(\% 95 \mathrm{Cl} ; 12-37 \%$ ) while in military women, it was equal to $25 \%$ (\% 95 Cl; 31-40\%) (Table 3).

36 cross-sectional studies had used the diagnostic and statistical manual of mental disorders-IV (DSM-IV), 24 studies had used the patient health questionnaire (PHQ), 13 studies had applied interviews using clinical criteria and symptoms, 5 studies had applied the Zung self-tool report depression scale (SDS), 9 studies had used the beck depression inventory (BDI) and 7 studies had used the center for epidemiological studies depression (CES-D) to diagnose depression in the active or in-service military. The overall prevalence of depression according to the diagnostic and statistical manual of mental disorders-IV (DSM-IV) was 15\% (\% $95 \mathrm{Cl} ; 17-35 \%$ ), according to the patient health questionnaire (PHQ), it was 15\% (\% $95 \mathrm{Cl} ; 13-17 \%)$, And according to the Zung self-report depression scale (SDS), it was equal to $20 \%$ (\% $95 \mathrm{Cl} ; 14-26 \%)$. Also, the overall pooled 
prevalence based on beck depression inventory (BDI) and the center for epidemiological studies depression (CES-D) was 25\% (\% 95 Cl; $15-36 \%$ ) and $13 \%$ (\% $95 \mathrm{Cl} ; 8-19 \%)$, respectively (Table 3).

\section{Publication bias, and meta-regression in studies related to the active military}

The results of the publication bias were shown in Fig. 2 for studies related to the active military. The results of the Eggers test showed that diffusion bias did not occur in calculating the prevalence of depression in the active military (B: 0.96, SE: 0.69, P: 0.167) (Fig. 2). In meta-regression analysis, the effect of military personnel age on prevalence was studied and analyzed. The results presented that age had a significant effect on the prevalence of depression in the active military and for every 1 year of age, depression increased by $0.04 \%$. The results of heterogeneity evaluation demonstrated that 5 studies were the cause of heterogeneity in the meta-analysis of the depression prevalence in active military (Fig. 2).

\section{Prevalence of depression in veterans}

Regarding the prevalence of depression in veterans, 80 cross-sectional articles with a sample size of 887982 people were reviewed, of whom 822967 people were depressed. After combining the results of these studies, the overall pooled prevalence of depression in veterans was $20 \%$ (\% 95 Cl; 18-22\%). The percentage of heterogeneity was $99.80 \%$ which was statistically significant (Table 3 ).

The results of the subgroup analysis showed that 55 studies had used the convinces sampling method and 25 studies had used the random sampling method to determine the prevalence of depression in veterans. The sample size in the studies that had used the convinces sampling method was equal to 565979 people. After combining their results, the pooled prevalence of depression was equal to 19\% (\% $95 \mathrm{Cl}$; 16-21 \%). Also, the sample size in studies that had used the random sampling method was equal to 32,2003 people. After combining their results, the pooled prevalence of depression in veterans was equal to $22 \%$ (\% $95 \mathrm{Cl} ; 18-27 \%$ ) (Table 3$)$.

Regarding the military community of different divisions, the analysis showed that in the case of veterans, 68 studies had been conducted in the veterans' community of the Army, and 12 studies had been conducted in the entire military (without separating the different divisions). There was no study in the Air Force or Navy. The sample size in military veterans was 583048 people and after combining these results, the pooled prevalence of depression was 19\% (\% $95 \mathrm{Cl}$; 17-22 \%) (Table 3).

The results of meta-analysis based on questionnaires and various measurement tools showed that heterogeneity of pooled prevalence was significantly reduced. In this section, 7 cross-sectional studies included in the meta-analysis using the beck depression inventory (BDI) questionnaire, 11 studies using the center for epidemiological studies depression (CES-D), 13 studies based on clinical criteria and interviews, 11 studies based on diagnostic and statistical manual of mental disorders-IV (DSM-IV), 29 studies based on the patient health questionnaire (PHQ), 2 studies based on the Zung self-report depression scale (SDS), 4 studies based on mental health inventory (MHI), 1 study based on Hamilton depression rating scale (HDRS), 1 study based on the quick inventory of depressive symptomatology (QIDS), and 1 study based on the geriatric depression scale (GDS) had examined depression in veterans. The results of the meta-analysis showed that the prevalence of depression according to the statistical manual of mental disorders-IV (DSM-IV), the patient health questionnaire (PHQ), and beck depression inventory (BDI) was 15\% (\% $95 \mathrm{Cl} ; 9-22 \%), 21 \%$ (\% $95 \mathrm{Cl} ; 17-25 \%$ ), and 14\% (\% $95 \mathrm{Cl} ; 9-21 \%$ ), respectively (Table 3).

\section{Publication bias, and meta-regression in studies related to veterans}

The results of the publication bias were shown in Fig. 2 for studies related to veterans. The results of the Eggers test presented that bias occurred in calculating the prevalence of depression in veterans (B: 8.95, SE: 0.54, P: 0.001) (Fig. 2). In meta-regression analysis, the effect of military age on prevalence was examined and analyzed, which showed that age did not have a significant effect on the prevalence of depression in military veterans.

\section{Prevalence of suicide in the military}

The results of this study demonstrated that 49 studies related to the prevalence of suicidal ideation in the military and 42 studies related to the prevalence of suicide attempts in the military were included in the meta-analysis. The sample size in studies related to suicidal ideation was 759,374 people, of whom a total of 20,065 individuals had suicidal ideation. However, the sample size in studies related to suicide attempts was equal to 438,890 people, of whom 5471 people had attempted suicide. The results of meta-analysis showed that the pooled prevalence of suicidal ideation in the entire military was $11 \%$ (\% $95 \mathrm{Cl}$; 10-13\%) (Fig. 3). The pooled prevalence of suicide attempts in all military was equal to the prevalence of suicidal ideation $11 \%$ (\% $95 \mathrm{Cl} ; 9-13 \%$ ) (Fig. 4).

To accurately estimate the prevalence of suicidal ideation in the military and to find the source of heterogeneity in the study, the subgroup analysis was performed based on whether the military person was serving or a veteran at that time, the study sampling method (random or convinces), the military service location, the statistical population of the study in terms of the presence of various diseases or being healthy, gender, and finally the tools used to measure suicide ideation and attempts. The results were shown in Table 4 . As can be seen from the results, the pooled prevalence of suicidal ideation in veterans was higher than that in active military (14\% vs. $10 \%)$. Suicidal ideation was also higher in women than men (Table 4 ). The pooled prevalence of suicidal ideation was higher in the air force (19\%) than that in the navy and the army (Table 4). In the military with substance use, the prevalence of suicidal ideation was $18 \%$ (\% $95 \mathrm{Cl} ; 7-33 \%)$, which was higher than one in the military consuming alcohol with a prevalence of $9 \%$ (\% $95 \mathrm{Cl} ; 4-13 \%$ ) (Table 4). In studies that had used multi-problem screening inventory (MPSI) and the self-injurious thoughts

Page 28/50 
and behaviors interview (SITBI) to estimate suicidal ideation, the prevalence was 39\% (\% $95 \mathrm{Cl}$; 36-41 \%), and 36\% (\% $95 \mathrm{Cl}$; $32-41 \%$ ), respectively, which was higher than those in studies that had used other tools to estimate the prevalence of suicidal ideation in the military (Table 4). 
Table 4

The pooled estimate of prevalence of suicide in active duty and veteran military

\begin{tabular}{|c|c|c|c|c|c|c|c|}
\hline \multirow[t]{2}{*}{ Categories } & \multirow[t]{2}{*}{$\begin{array}{l}\text { No. of Studies (Sample } \\
\text { Size) }\end{array}$} & \multirow[t]{2}{*}{$\begin{array}{l}\text { Pooled Prevalence (\% } 95 \\
\mathrm{Cl})\end{array}$} & \multicolumn{3}{|c|}{$\begin{array}{l}\text { Between studies heterogeneity } \\
\text { assessment (\%) }\end{array}$} & \multicolumn{2}{|c|}{$\begin{array}{l}\text { Between subgroups } \\
\text { heterogeneity } \\
\text { assessment (\%) }\end{array}$} \\
\hline & & & $R$ & $P_{\text {Heterogenity }}$ & $Z$ & Q & $P_{\text {Heterogenity }}$ \\
\hline \multicolumn{8}{|c|}{ The prevalence of suicide thought in military } \\
\hline Military Statue & $31(424253)$ & $10 \%(7-13 \%)$ & \multirow{2}{*}{$\begin{array}{l}67.55 \\
\% \\
69.77 \\
\%\end{array}$} & 0.402 & 12.55 & \multirow[t]{2}{*}{2.24} & \multirow[t]{2}{*}{0.130} \\
\hline $\begin{array}{l}\text { Active Duty } \\
\text { Veteran }\end{array}$ & $18(335121)$ & $14 \%(10-20 \%)$ & & 0.329 & 9.59 & & \\
\hline Sampling Method & $40(151199)$ & $12 \%(10-15 \%)$ & \multirow{2}{*}{$\begin{array}{l}57.23 \\
\% \\
74.31 \\
\%\end{array}$} & 0.170 & 20.37 & \multirow[t]{2}{*}{15.76} & \multirow[t]{2}{*}{0.001} \\
\hline $\begin{array}{l}\text { Convinces Sampling } \\
\text { Random Sampling }\end{array}$ & $9(608175)$ & $7 \%(6-9 \%)$ & & 0.059 & 17.83 & & \\
\hline Type of Forces & $2(441)$ & $19 \%(15-22 \%)$ & \multirow{3}{*}{$\begin{array}{l}78.99 \\
\% \\
88.68 \\
\%\end{array}$} & 0.041 & 17.36 & \multirow[t]{5}{*}{30.05} & \multirow[t]{5}{*}{0.001} \\
\hline Air Forces & $23(434677)$ & $8 \%(5-11 \%)$ & & 0.025 & 9.57 & & \\
\hline Armed Forces & 2 (295715) & $1 \%(1-2 \%)$ & & 0.0001 & 10.98 & & \\
\hline Marine Forces & $22(28982)$ & $16 \%(12-21 \%)$ & $\begin{array}{l}93.86 \\
\%\end{array}$ & 0.041 & 13.22 & & \\
\hline Military Forces & & & \multicolumn{3}{|l|}{$\begin{array}{l}77.83 \\
\%\end{array}$} & & \\
\hline Population & 42 (757597) & $11 \%(9-13 \%)$ & $\begin{array}{l}99.80 \\
\%\end{array}$ & 0.0001 & 20.07 & \multirow[t]{4}{*}{1.74} & \multirow[t]{4}{*}{0.420} \\
\hline Healthy Forces & - & - & & - & - & & \\
\hline Forces with HIV/AIDS & $1(93)$ & $9 \%(4-13 \%)$ & & - & 4.45 & & \\
\hline $\begin{array}{l}\text { Forces with Alcohol Use } \\
\text { Forces with Substance } \\
\text { Use }\end{array}$ & $6(1684)$ & $18 \%(7-33 \%)$ & $\begin{array}{l}97.74 \\
\%\end{array}$ & 0.0001 & 4.91 & & \\
\hline Gender & $44(756218)$ & $11 \%(9-13 \%)$ & \multirow{3}{*}{$\begin{array}{l}\begin{array}{l}88.72 \\
\%\end{array} \\
90.00 \\
\%\end{array}$} & 0.0001 & 20.10 & \multirow[t]{4}{*}{12.30} & \multirow[t]{4}{*}{0.001} \\
\hline Total & $2(1532)$ & $14 \%(12-16 \%)$ & & 0.0001 & 10.94 & & \\
\hline Male & $3(1624)$ & $20 \%(14-27 \%)$ & & 0.017 & 28.78 & & \\
\hline Female & & & $\begin{array}{l}75.22 \\
\%\end{array}$ & & & & \\
\hline
\end{tabular}

Beck Scale for Suicidal Ideation-Current (BSSI-C), Brief self-report questionnaire (SCRENNER), SCID DSM-IV Diagnoses, Multi-Problem Screening Inventory (MPSI), The 4-item Suicidal Behaviors Questionnaire-Short Form (SBQ-SF), The Patient Health Questionnaire(PHQ), The Self-Injurious Thoughts and Behaviors Interview (SITBI), The Suicidal Behaviors Questionnaire Revised (SBQ-R), The Columbia Suicidal Severity Rating Scale (C-SSRS), the Mini International Neuropsychiatric Interview Plus (MINI-Plus) 


\begin{tabular}{|c|c|c|c|c|c|c|c|}
\hline \multirow[t]{2}{*}{ Categories } & \multirow[t]{2}{*}{$\begin{array}{l}\text { No. of Studies (Sample } \\
\text { Size) }\end{array}$} & \multirow[t]{2}{*}{$\begin{array}{l}\text { Pooled Prevalence (\% } 95 \\
\mathrm{Cl})\end{array}$} & \multicolumn{3}{|c|}{$\begin{array}{l}\text { Between studies heterogeneity } \\
\text { assessment (\%) }\end{array}$} & \multicolumn{2}{|c|}{$\begin{array}{l}\text { Between subgroups } \\
\text { heterogeneity } \\
\text { assessment (\%) }\end{array}$} \\
\hline & & & $R$ & $P_{\text {Heterogenity }}$ & $z$ & Q & $P_{\text {Heterogenity }}$ \\
\hline Tools & $5(12775)$ & $11 \%(7-16 \%)$ & \multirow{2}{*}{$\begin{array}{l}67.96 \\
\%\end{array}$} & 0.052 & 8.98 & \multirow[t]{9}{*}{24.84} & 0.001 \\
\hline BSSI-C Scale & $1(669)$ & $8 \%(6-10 \%)$ & & - & 13.54 & & \\
\hline SCRENNER Scale & $16(375640)$ & $7 \%(5-10 \%)$ & \multirow{2}{*}{$\begin{array}{l}69.80 \\
\%\end{array}$} & 0.049 & 10.23 & & \\
\hline SCID DSM-IV Scale & $2(1225)$ & $39 \%(36-41 \%)$ & & 0.077 & 15.52 & & \\
\hline MPSI Scale & $15(324540)$ & $9 \%(6-13 \%)$ & \multirow{3}{*}{$\begin{array}{l}55.21 \\
\% \\
53.01 \\
\%\end{array}$} & 0.850 & 9.61 & & \\
\hline PHQ Scale & $2(443)$ & $36 \%(32-41 \%)$ & & 0.501 & 13.35 & & \\
\hline SITBI Scale & $7(5845)$ & $16 \%(14-18 \%)$ & & 0.053 & 12.25 & & \\
\hline SBQ-R Scale & $1(38237)$ & $14 \%(12-18 \%)$ & 44.34 & - & 14.98 & & \\
\hline C-SSRS Scale & & & \multicolumn{3}{|l|}{$\begin{array}{l}77.69 \\
\%\end{array}$} & & \\
\hline \multicolumn{8}{|c|}{ The prevalence of suicide attempted in military } \\
\hline Military Statue & $19(98426)$ & $8 \%(6-10 \%)$ & \multirow{3}{*}{$\begin{array}{l}50.18 \\
\% \\
69.80 \\
\%\end{array}$} & 0.497 & 12.14 & \multirow[t]{3}{*}{10.13} & \multirow[t]{3}{*}{0.001} \\
\hline Active Duty & $23(340464)$ & $15 \%(11-19 \%)$ & & 0.122 & 12.59 & & \\
\hline Veteran & & & & & & & \\
\hline Sampling Method & 35 (133437) & $11 \%(9-13 \%)$ & \multirow{3}{*}{$\begin{array}{l}77.78 \\
\% \\
64.26 \\
\%\end{array}$} & 0.059 & 16.11 & \multirow[t]{3}{*}{0.30} & \multirow[t]{3}{*}{0.660} \\
\hline Convinces Sampling & $7(305453)$ & $13 \%(7-20 \%)$ & & 0.051 & 6.47 & & \\
\hline Random Sampling & & & & & & & \\
\hline Type of Forces & $4(4851)$ & $13 \%(1-35 \%)$ & \multirow{3}{*}{$\begin{array}{l}79.99 \\
\% \\
76.44 \\
\%\end{array}$} & 0.047 & 2.54 & \multirow[t]{5}{*}{1.27} & \multirow[t]{5}{*}{0.260} \\
\hline Air Forces & $23(121644)$ & $12 \%(9-15 \%)$ & & 0.044 & 14.50 & & \\
\hline Armed Forces & $1(100)$ & $54 \%(44-64 \%)$ & & - & 15.54 & & \\
\hline Marine Forces & $14(312295)$ & $8 \%(4-12 \%)$ & - & 0.034 & 6.82 & & \\
\hline Military Forces & & & $\begin{array}{l}74.77 \\
\%\end{array}$ & & & & \\
\hline
\end{tabular}

Beck Scale for Suicidal Ideation-Current (BSSI-C), Brief self-report questionnaire (SCRENNER), SCID DSM-IV Diagnoses, Multi-Problem Screening Inventory (MPSI), The 4-item Suicidal Behaviors Questionnaire-Short Form (SBQ-SF), The Patient Health Questionnaire(PHQ), The Self-Injurious Thoughts and Behaviors Interview (SITBI), The Suicidal Behaviors Questionnaire Revised (SBQ-R), The Columbia Suicidal Severity Rating Scale (C-SSRS), the Mini International Neuropsychiatric Interview Plus (MINI-Plus) 


\begin{tabular}{|c|c|c|c|c|c|c|c|}
\hline \multirow[t]{2}{*}{ Categories } & \multirow[t]{2}{*}{$\begin{array}{l}\text { No. of Studies (Sample } \\
\text { Size) }\end{array}$} & \multirow[t]{2}{*}{$\begin{array}{l}\text { Pooled Prevalence (\% } 95 \\
\mathrm{Cl} \text { ) }\end{array}$} & \multicolumn{3}{|c|}{$\begin{array}{l}\text { Between studies heterogeneity } \\
\text { assessment (\%) }\end{array}$} & \multicolumn{2}{|c|}{$\begin{array}{l}\text { Between subgroups } \\
\text { heterogeneity } \\
\text { assessment (\%) }\end{array}$} \\
\hline & & & $R$ & $P_{\text {Heterogenity }}$ & $Z$ & Q & $P_{\text {Heterogenity }}$ \\
\hline Population & $35(435640)$ & $9 \%(8-11 \%)$ & \multirow{2}{*}{$\begin{array}{l}99.09 \\
\%\end{array}$} & 0.0001 & 18.52 & \multirow[t]{6}{*}{84.99} & \multirow[t]{6}{*}{0.001} \\
\hline Healthy Forces & $1(442)$ & $5 \%(4-8 \%)$ & & - & 19.33 & & \\
\hline Forces with HIV/AIDS & $1(1210)$ & $8 \%(7-10 \%)$ & \multirow{3}{*}{$\begin{array}{l}- \\
87.44\end{array}$} & - & 14.59 & & \\
\hline $\begin{array}{l}\text { Forces with Alcohol Use } \\
\text { Forces with Substance } \\
\text { Use }\end{array}$ & $5(1598)$ & $30 \%(23-36 \%)$ & & 0.0001 & 8.99 & & \\
\hline \multirow[t]{2}{*}{ Forces with HCV } & & & & & & & \\
\hline & - & - & - & - & - & & \\
\hline Gender & 37 (429113) & $11 \%(9-13 \%)$ & \multirow{3}{*}{$\begin{array}{l}92.88 \\
\% \\
95.91 \\
\%\end{array}$} & 0.0001 & 9.04 & \multirow[t]{4}{*}{9.56} & \multirow[t]{4}{*}{0.001} \\
\hline Total & $2(4533)$ & $3 \%(2-4 \%)$ & & 0.0001 & 2.49 & & \\
\hline Male & \multirow[t]{2}{*}{$3(5244)$} & \multirow[t]{2}{*}{$21 \%(1-53 \%)$} & & 0.0001 & 10.75 & & \\
\hline Female & & & $\begin{array}{l}98.49 \\
\%\end{array}$ & & & & \\
\hline Tools & $6(9800)$ & $15 \%(10-22 \%)$ & \multirow{3}{*}{$\begin{array}{l}66.33 \\
\% \\
78.31 \\
\%\end{array}$} & 0.050 & 9.04 & \multirow[t]{13}{*}{35.33} & \multirow[t]{13}{*}{0.001} \\
\hline BSSI-C Scale & $6(6882)$ & $11 \%(5-20 \%)$ & & 0.049 & 5.25 & & \\
\hline Checklist & $12(373059)$ & $5 \%(3-7 \%)$ & & 0.041 & 11.15 & & \\
\hline SCID DSM-IV Scale & $2(1225)$ & $36 \%(33-38 \%)$ & \multirow{3}{*}{$\begin{array}{l}61.99 \\
\% \\
55.99 \\
\%\end{array}$} & 0.055 & 43.39 & & \\
\hline MPSI Scale & $4(4675)$ & $9 \%(5-15 \%)$ & & 0.039 & 6.54 & & \\
\hline PHQ Scale & $2(2417)$ & $4 \%(4-5 \%)$ & & 0.041 & 19.36 & & \\
\hline MINI-Plus Scale & $3(526)$ & $11 \%(1-49 \%)$ & \multirow{3}{*}{$\begin{array}{l}70.05 \\
\% \\
60.44 \\
\%\end{array}$} & 0.050 & 1.42 & & \\
\hline SBQ-R Scale & $1(374)$ & $8 \%(5-11 \%)$ & & - & 10.00 & & \\
\hline SITBI Scale & $2(38330)$ & $1 \%(1-2 \%)$ & & 0.034 & 32.48 & & \\
\hline C-SSRS Scale & $3(692)$ & $22 \%(2-53 \%)$ & $\begin{array}{l}69.01 \\
\%\end{array}$ & 0.045 & 2.76 & & \\
\hline \multirow{3}{*}{ NR } & & & \multicolumn{3}{|l|}{ - } & & \\
\hline & & & \multicolumn{3}{|l|}{$\begin{array}{l}62.99 \\
\%\end{array}$} & & \\
\hline & & & \multicolumn{3}{|l|}{$\begin{array}{l}79.90 \\
\%\end{array}$} & & \\
\hline
\end{tabular}

In terms of the prevalence of suicide attempts, servicemen serving in the air force were more likely to commit suicide than ones in the army (13\% vs. $12 \%$ ). In the present analysis, the prevalence of suicide attempts in the navy was $54 \%$, but this was the result of a study with a sample size of 100 people that could not be trusted and compared with the prevalence of suicide attempts in other military (Table 4).

The prevalence of suicide attempts in militaries with substance use was $30 \%(\% 95 \mathrm{Cl} ; 23-36 \%)$, which was higher than the prevalence of suicide attempts in non-drug-using military. Also, the prevalence of suicide attempts was $8 \%$ in militaries consuming alcohol (\% $95 \mathrm{Cl} ; 7-10 \%)$ and in 
militaries with AIDS / HIV, it was equal to $5 \%$ (\% $95 \mathrm{Cl}$; 4-8\%) (Table 4). Also, suicide attempts in female soldiers was more than that in male soldiers (21\% vs. $3 \%)$ (Table 4$)$.

The prevalence of suicide attempts was also analyzed based on the tools used in the studies. The results showed that after combining studies using SCID DSM-IV diagnoses, beck scale for suicidal ideation-current (BSSI-C), multi-problem screening inventory (MPSI) and the suicidal behavior questionnaire revised (SBQ-R), the prevalence was 5\% (\% $95 \mathrm{Cl} ; 3-7 \%), 15 \%$ (\% $95 \mathrm{Cl} ; 10-22 \%) 36 \%$ (\% $95 \mathrm{Cl} ; 33-38 \%$ ), $11 \%$ (\% $95 \mathrm{Cl}$; $1-49 \%$ ), respectively (Table 4).

\section{Publication bias, and meta-regression in studies related to the spread of suicide ideation and attempts}

The results of the diffusion bias were shown in Fig. 5. The results of the Eggers test represented that diffusion bias occurred in calculating the prevalence of suicidal ideation (B: 7.59, SE: 0.99, P: 0.001) and suicide attempts (B: 7.03, SE: 0.44, P: 0.001) in the military (Fig. 5). In metaregression analysis, the effect of military age on prevalence was examined and analyzed. The results showed that age did not have a significant effect on the prevalence of suicidal ideation and suicide attempts in the military.

\section{Discussion}

The present study was a systematic review and meta-analysis that showed that the pooled prevalence of depression in the active military was $23 \%$. According to the World Health Organization, the prevalence of depression in the general population is 15 to $20 \%$ ( 30,31$)$. Therefore, it can be said that the prevalence of depression in the military community is higher than that in the general community. Feeling sad in unfavorable situations such as military situations and operational locations can be one of the reasons for the increase in the prevalence of depression or in some way the occurrence of depression and its symptoms in the military. This relationship indicates the existence of a relation between activity abnormalities, mood and thoughts with social or occupational environments (32-36). On the other hand, the military may not be very interested in their job and, therefore, they have unpleasant moods and thoughts such as sadness, grief, despair and worry, which can make a military person prone to depression $(37,38)$. Military personnel often suffer from disorders in sleep, nutrition, physical exertion, concentration, as well as anorexia, and weight changes due primarily to job sensitivity and confidential activities. The presence of these behaviors and emotions over time and their stability for a long time have a negative effect on the mood of these people and can expose a military person to depression (39, 40). In the present meta-analysis, the pooled prevalence of depression after combining studies in which the available sampling method had been used, was equal to $21 \%$ and after combining studies that had used random sampling method to collect their samples, the pooled prevalence of depression was equal to $26 \%$. In cross-sectional studies, the sampling method should be random in order to consider samples under investigation as a good representative of the target population. In studies that had selected this type of sampling, the pooled prevalence of depression was higher. On the other hand, the results of the subgroup analysis showed that the amount of heterogeneity after the analysis based on the sampling method has decreased, which indicated that different sampling methods in meta-analysis studies were one of the sources of heterogeneity in the total pooled prevalence in the active military.

The results of the present meta-analysis represented that the prevalence of depression was higher in active servicemen in the navy than in those in the air force and the army. The navy has more professional problems in terms of special professional missions, and more psychological problems than the army and the air Force. Job-related stress, complex missions, strict rules, the possibility of injury, disability, captivity and even death are some of the issues that increase the likelihood of depression in these soldiers compared to others (41, 42). A person's psychological capacity includes a person's ability to cope with the expectations and difficulties of everyday life. High psychological capacity allows a person to maintain his/her life at the desired psychological level and crystallize this ability in the form of adaptive behaviors, effective and positive actions for himself/herself. The role of psychological capacity in promoting health and well-being in all three aspects of physical, mental and social is very important. This importance becomes even more apparent when the problem becomes behavioral. In such a case, the person is not strong enough when faced with psychological pressures and obstacles in life, and as a result, his/her inappropriate behavior will be the source of all suffering and failure $(43,44)$. Therefore, addressing various psychological aspects, quality of life and social relations of the military, especially the navy, in order to properly understand the conditions of these people and their families can be useful to strengthen and enhance their military capabilities and efficiency. Other reasons for the increasing prevalence of depression in the navy include family problems (45). Over the years, research has shown that the family plays an important role in providing function and activity to individuals. Having a healthy society depends on having strong families in the society. Navy families often suffer from the stress of being away from a normal life, living in unfamiliar environments, and experiencing life outside their homelands. These may cause problems within the family, which ultimately reduce the ability of the navy and cause psychological problems such as depression $(46,47)$.

The stress of military jobs has major and significant consequences for the family environment. Psychological disorders between military families have been reported between 3 to 15\% depending on the disorder type, while they have been reported paranoid disorders, obsessive-compulsive disorders, depression, interpersonal relationships, physical problems, and aggression, respectively (47). According to research, it has been shown that the prevalence of these disorders in military families was higher than that in other families in the society. Factors such as workplace stress, sensitive and critical situations, high job responsibilities, job stress, unwanted relocation, problems in the family and home, lack of confidence in

Page 33/50 
individual abilities, mental fatigue caused by hard work, thinking the possibility of death are some of the depression and mental distress causes in the military and their families $(48,49)$. In a study entitled Environment, Lifestyle and Psychological Factors in the Health and Welfare of Military Families, the results showed that the psychological factors resulting from military missions were divided into 5 stages which included the stage before deployment, deployment, return, reinforcement and re-deployment, respectively. Military personnel and their families also experienced different psychological difficulties before, during, or after deployment to different missions. These experiences brought them many psychological norms that varied with different variables such as the location of the mission (in terms of the possibility of military conflict with hostile forces), duration of deployment, number of deployments, time between deployments, military responsibility, and the difficulty of working conditions of individuals at the time of deployment (50-52). The same factors may lead military personnel to use drugs, and alcohol (53). In the present metaanalysis, the prevalence of depression in the active military drug users was $37 \%$ and in the military alcohol users was $29 \%$. Drug, and alcohol abuse can be a contributing factor to depression or other mental disorders in the military. Excessive alcohol abuse in the US military has resulted in significant financial losses. Data from 2006 showed that excessive alcohol consumption annually cost the US military 1.12 billion dollars (54, 55). In a large survey study by Bray and Hourani, the results demonstrated that the prevalence of alcohol use in the US military was 15 to $20 \%$ (56). Also, in terms of gender, this prevalence was different and in men, alcohol consumption was 3.5 times more than that in military women. The results of studies have shown that the prevalence of alcohol and drug use in the Navy was higher than that in the Air Force, which might be related to the high prevalence of depression in the navy (57-59). Alcohol and substance abuse occur more frequently in war veterans. A study by Milliken and colleagues in a population-based study found that 12 to 15 percent of veterans experienced alcohol and substance abuse after 3 to 6 months of returning from war, which put them at risk of depression (60-62). In the present meta-analysis, the overall pooled prevalence of depression in veterans was $20 \%$. However, in studies that had used random sampling to collect samples, the prevalence was $22 \%$.

The prevalence of depression was 15\% in active HIV-positive servicemen and $16 \%$ in HIV-positive veterans. These military personnel, of course, suffered from depression and other mental disorders due to the existence of the disease and its difficult conditions in the society. The prevalence of depression in veterans with hepatitis C was $29 \%$. It was noteworthy that the amount of heterogeneity during the subgroup analysis based on the healthy and unhealthy military population did not significantly decrease compared to the overall prevalence of heterogeneity, which indicated the lack of the inclusion effect of soldiers with various diseases, and healthy soldiers on the amount of heterogeneity in studies. In other words, this factor could not be a source of heterogeneity when estimating the overall prevalence of depression. However, as shown in Table 4, the type of sampling (random or available), location and place of service (the air, naval or army), and various tools for measuring the prevalence of depression were the main sources of heterogeneity when estimating general depression in the military because the amount and percentage of heterogeneity had significantly decreased when performing subgroups based on these variables.

The prevalence of suicidal ideation in the present meta-analysis in the military was equal to the prevalence of suicide attempts in the entire military. Suicidal ideation was also more common in women than in military men. According to studies conducted in the world, the prevalence of suicide and its thoughts in the military had a range from $5.8 \%$ to $28.4 \%$, which in the present meta-analysis study was exactly equal to $11 \%$. In the study of Farsi et al., the results showed that with increasing scores of depression, the possibility of self-harm and suicide in the military increased (63). In the study by Hossieni et al., The prevalence of depressive disorders in military personnel who had attempted suicide was 0.7 to $1.3 \%$ (64). The prevalence of suicidal ideation was higher in Air Force servicemen than that in Navy and Land Force servicemen. The prevalence of suicidal ideation was $18 \%$ in the military using drug, which was higher than that in the military using alcohol. Also, the prevalence of suicide attempts in drug-using military was higher than the prevalence of suicide attempts in non-drug-using military. The results of the present meta-analysis showed that the use of drugs, alcohol and diseases such as HIV and HCV could be a predisposing factor in the development of mental disorders and the development of suicidal ideation and suicide attempts in the military. In addition, there were more thoughts and attempts to commit suicide in veterans than in active and serving soldiers. One of the effective reasons for the existence of suicidal ideation and attempts in the veterans was the lack of combat and other physical activities, living at home, consuming drugs and alcohol. The results of the present meta-analysis represented that the prevalence of suicidal ideation and attempts in military personnel using drugs were equal to $18 \%$ and $30 \%$, respectively.

Regarding the prevalence of suicidal ideation and attempts, the results of the subgroup analysis showed that the use of different tools in determining the prevalence of suicidal ideation in the military in meta-analysis studies, different sampling methods (available or random sampling), and the type of servicemen included in the study (in-service or veterans) were among the most important factors in creating heterogeneity in determining the pooled prevalence of suicidal ideation and attempts in the military after completing the entire study.

The present meta-analysis study was the first systematic review and meta-analysis study to determine the prevalence of depressive and suicidal disorders in the entire military worldwide. Also, the exact prevalence of these disorders in the military had not been reported and this research determined the overall pooled prevalence of depression and suicidal ideation or attempts. On the other hand, the sample size in the present metaanalysis subgroup was very significant, which made the estimated prevalence in each subgroup very reliable. Other benefits of this study included determining the prevalence of depressive disorders and suicide in military personnel in various sectors, such as the navy, air, and army forces. One of the limitations of the present study was the lack of sufficient number of studies and sample sizes to determine the prevalence of depressive and suicidal disorders in servicemen with hepatitis $\mathrm{C}$ or other diseases. For future research, the issue of social classes, religion, and income levels need to be considered to determine the prevalence of mental disorders in the military. Also, studies on how to carry out preventive interventions, and their cost-effectiveness need to be done in order to determine effective and useful interventions in the military to prevent suicide and depression. 


\section{Conclusion}

The present study showed that the prevalence of depression and suicide (thoughts and actions) was high in the military, especially in the navy and air forces, and this prevalence was more significant. On the other hand, substance and alcohol consumption were factors that increased the prevalence of depression and ultimately led to suicide in the military. Therefore, it is necessary to develop and design training and intervention programs in order to train and increase the awareness of the military, especially veterans, in order to prevent the occurrence of suicide and mental disorders such as depression. Considering the prevalence of depression and suicide in the military consuming drugs and alcohol in the present meta-analysis study, it is necessary to implement screening and follow-up measures to identify, and prevent these two disorders (drug and alcohol consumption) in the military.

\section{Abbreviations}

$\mathrm{Cl}$

Confidence Interval

EMBASE

Excerpta Medica dataBASE

NOS

Newcastle-Ottawa Scale

MOOSE

The Meta-Analyses of Observational Studies in Epidemiology

PRISMA

Preferred Reporting Items for Systematic Reviews and Meta-analyses

WHO

World Health Organization

DSM-IV

The diagnostic and statistical manual of mental disorders-IV

$\mathrm{PHQ}$

The patient health questionnaire

SDS

The Zung self-tool report depression scale

BDI

The beck depression inventory

CES-D

The center for epidemiological studies depression

HDRS

Hamilton depression rating scale

$\mathrm{MHI}$

Mental health inventory

QIDS

The quick inventory of depressive symptomatology

GDS

The geriatric depression scale

SCID DSM-IV

Structured Clinical Interview for DSM Disorders

BSSI-C

Beck scale for suicidal ideation-current

MPSI

Multi-problem screening inventory

SBQ-R

The suicidal behavior questionnaire revised

SCRENNER

Brief self-report questionnaire

MPSI

Multi-Problem Screening Inventory

SBQ-SF

The 4-item Suicidal Behaviors Questionnaire-Short Form 
The Self-Injurious Thoughts and Behaviors Interview

SBQ-R

The Suicidal Behaviors Questionnaire Revised

C-SSRS

The Columbia Suicidal Severity Rating Scale

MINI-Plus

The Mini International Neuropsychiatric Interview Plus

BSI

The Brief Symptom Inventory

HADS

The Hospital Anxiety and Depression Scale

GDS

Geriatric Depression Scale

QIDS

Quick Inventory of Depressive Symptomatology

\section{Declarations}

\section{Ethics approval and consent to participate}

Not applicable because no primary data were collected.

\section{Consent for Publication}

Not applicable.

Availability of data and material

Data is available and it can be accessed from the corresponding author with reasonable inquiry.

\section{Competing interests}

The authors declare that they have no competing interests.

\section{Funding}

None.

\section{Competing interests}

The authors declare that they have no competing interests.

\section{Funding}

None.

\section{Authors' contributions}

YM, BD, and MS conceptualized the idea for this review, formulated the review question, and objectives. All authors contributed equally to the formulation of the development of the search strategy, conducting the searches, data extraction, data analysis/interpretation, and writing the manuscript. All authors read and approved the final manuscript.

\section{Acknowledgments}

Not applicable

\section{References}

1. Wise T, Dolan RJ. Associations between aversive learning processes and transdiagnostic psychiatric symptoms in a general population sample. Nat Commun. 2020;11(1):4179. 
2. Best MW, Law H, Pyle M, Morrison AP. Relationships between psychiatric symptoms, functioning and personal recovery in psychosis. Schizophrenia research. 2020;223:112-8.

3. Foxx CL, Heinze JD, González A, Vargas F, Baratta MV, Elsayed Al, et al. Effects of Immunization With the Soil-Derived Bacterium Mycobacterium vaccae on Stress Coping Behaviors and Cognitive Performance in a "Two Hit" Stressor Model. Front Physiol. 2020;11:524833.

4. Mota N, Bolton SL, Enns MW, Afifi TO, El-Gabalawy R, Sommer JL, et al. Course and Predictors of Posttraumatic Stress Disorder in the Canadian Armed Forces: A Nationally Representative, 16-Year Follow-up Study: Cours et prédicteurs du trouble de stress post-traumatique dans les Forces armées canadiennes: une étude de suivi de 16 ans nationalement représentative. Canadian journal of psychiatry Revue canadienne de psychiatrie. 2021:706743721989167.

5. Sullivan KS, Hawkins SA, Gilreath TD, Castro CA. Mental health outcomes associated with profiles of risk and resilience among U.S. Army spouses. Journal of family psychology : JFP : journal of the Division of Family Psychology of the American Psychological Association (Division 43). 2020.

6. Marini CM, Fiori KL, Wilmoth JM, Pless Kaiser A, Martire LM. Psychological Adjustment of Aging Vietnam Veterans: The Role of Social Network Ties in Reengaging with Wartime Memories. Gerontology. 2020;66(2):138-48.

7. Vogt DS, Tyrell FA, Bramande EA, Nillni YI, Taverna EC, Finley EP, et al. U.S. Military Veterans' Health and Well-Being in the First Year After Service. American journal of preventive medicine. 2020;58(3):352-60.

8. Herzog S, Fogle BM, Harpaz-Rotem I, Tsai J, Pietrzak RH. Dissociative Symptoms in a Nationally Representative Sample of Trauma-Exposed U.S. Military Veterans: Prevalence, Comorbidities, and Suicidality. Journal of affective disorders. 2020;272:138-45.

9. Brockelmeyer MC. The Impact of Military Culture on Veterans Entering the Private Workforce: A Qualitative Ethnographic Study: Northcentral University; 2020.

10. Wilcove GL, Schwerin MJ, Wolosin DG. An exploratory model of quality of life in the US Navy. Military Psychology. $2003 ; 15(2): 133-52$.

11. Hindelang RL, Schwerin MJ, Farmer WL. Quality of life (QOL) in the US Marine Corps: The validation of a QOL model for predicting reenlistment intentions. Military Psychology. 2004;16(2):115-34.

12. Sticha PJ, Sadacca R, DiFazio AS, Knerr CM, Hogan PF. Personnel Tempo: Definition, Measurement, and Effects on Retention, Readiness, and Quality of Life. HUMAN RESOURCES RESEARCH ORGANIZATION ALEXANDRIA VA; 1999.

13. Levina M, Krieger E. Interrelation and Interdependence of Attitudes of Choice of Life Path and Propensity to Deviant Behavior in Military Personnel. Psychological-Educational Studies. 2020;12(3):137-51.

14. Dunlap SL, Holloway IW, Pickering CE, Tzen M, Goldbach JT, Castro CA. Support for transgender military service from active duty United States military personnel. Sexuality Research and Social Policy. 2021;18(1):137-43.

15. Rabin JS. Psychological Mechanisms Underlying the Populist Threat to Democracy. The Psychology of Political Behavior in a Time of Change: Springer; 2021. p. 473-527.

16. Reyes ME, Dillague SGO, Fuentes MIA, Malicsi CAR, Manalo DCF, Melgarejo JMT, et al. Self-Esteem and Optimism as Predictors of Resilience among Selected Filipino Active Duty Military Personnel in Military Camps. Journal of Positive School Psychology. 2020;4(1):15-25.

17. Moody RL, Savarese E, Gurung S, Rendina HJ, Parsons JT. The mediating role of psychological distress in the association between harassment and alcohol use among lesbian, gay, and bisexual military personnel. Substance use \& misuse. 2020;55(12):2055-63.

18. Van Den Berk-Clark C, Balan S, Shroff MV, Widner G, Price RK. The impact of hazardous alcohol use on behavioral healthcare utilization among National Guard service members. Substance use \& misuse. 2016;51(5):625-36.

19. Kelley ML, Milletich RJ, Hollis BF, Veprinsky A, Robbins AT, Snell AK. Social support and relationship satisfaction as moderators of the stressmood-alcohol link association in US Navy members. The Journal of nervous and mental disease. 2017;205(2):99-105.

20. Coronges KA, Miller KA, Tamayo Cl, Ender MG. A network evaluation of attitudes toward gays and lesbians among US military cadets. Journal of homosexuality. 2013;60(11):1557-80.

21. Cadigan JM, Klanecky AK, Martens MP. An examination of alcohol risk profiles and co-occurring mental health symptoms among OEF/OIF veterans. Addictive behaviors. 2017;70:54-60.

22. Smith BM, Twohy AJ, Smith GS. Psychological inflexibility and intolerance of uncertainty moderate the relationship between social isolation and mental health outcomes during COVID-19. Journal of Contextual Behavioral Science. 2020;18:162-74.

23. Presti G, Dal Lago B, Fattori A, Mioli G, Moderato P, Sciaretta L, et al. Mental health support to staff in a major hospital in Milan (Italy) during the COVID-19 pandemic: a framework of actions. General Psychiatry. 2020;33(4).

24. McCracken LM, Badinlou F, Buhrman M, Brocki KC. Psychological impact of COVID-19 in the Swedish population: Depression, anxiety, and insomnia and their associations to risk and vulnerability factors. European Psychiatry. 2020;63(1).

25. Stroup DF, Berlin JA, Morton SC, Olkin I, Williamson GD, Rennie D, et al. Meta-analysis of observational studies in epidemiology: a proposal for reporting. Meta-analysis Of Observational Studies in Epidemiology (MOOSE) group. Jama. 2000;283(15):2008-12.

26. Moher D, Liberati A, Tetzlaff J, Altman DG. Preferred reporting items for systematic reviews and meta-analyses: the PRISMA statement. Int J Surg. 2010;8(5):336-41.

Page $37 / 50$ 
27. Moher D, Liberati A, Tetzlaff J, Altman DG, Group P. Preferred reporting items for systematic reviews and meta-analyses: the PRISMA statement. PLoS medicine. 2009;6(7):e1000097.

28. Stang A. Critical evaluation of the Newcastle-Ottawa scale for the assessment of the quality of nonrandomized studies in meta-analyses. European journal of epidemiology. 2010;25(9):603-5.

29. Wells GA, Shea B, O'Connell Da, Peterson J, Welch V, Losos M, et al. The Newcastle-Ottawa Scale (NOS) for assessing the quality of nonrandomised studies in meta-analyses. Oxford; 2000.

30. Zandi A, Sayari R, Ebadi A, Sanainasab H. Abundance of depression, anxiety and stress in militant Nurses. Journal Mil Med. 2011;13(2):103-8.

31. Mohammadi M, Vaisi-Raygani AA, Jalali R, Salari N, Sabbaghchi M. Prevalence of Depression in Nurses Working in Iranian Hospitals: A Systematic Review and Meta-analysis. International Journal of Medical Reviews. 2020;7(1):32-8.

32. Groll DL, Ricciardelli R, Carleton RN, Anderson G, Cramm H. A cross-sectional study of the relationship between previous military experience and mental health disorders in currently serving public safety personnel in Canada. The Canadian Journal of Psychiatry. 2020;65(5):330-7.

33. Rowan AB, Travis WJ, Richardson CB, Adams TR. Military Mental Health Personnel Deployment Survey: A Secondary Analysis. Military medicine. 2020;185(3-4):e340-e6.

34. Rugo KF, Tabares JV, Crowell SE, Baucom BR, Rudd MD, Bryan CJ. The role of depression and suicidal cognitions as contributors to suicide risk among active duty soldiers reporting symptoms of posttraumatic stress disorder. Journal of affective disorders. 2020;265:333-41.

35. Sadler N, Forbes D, O’Donnell M. Mental Health, Well-being, and Suicidality Following Separation from the Military: Advancing Research and Practice. Psychiatry. 2020;83(2):176-8.

36. Wang J, Ursano RJ, Gifford RK, Dinh H, Farooq S, Broshek CE, et al. Mental health and suicidality in separating US Reserve and National Guard personnel. Psychiatry. 2020;83(2):166-75.

37. Hu Y, Chu X, Urosevich TG, Hoffman SN, Kirchner HL, Adams RE, et al. Predictors of current DSM-5 PTSD diagnosis and symptom severity among deployed veterans: significance of predisposition, stress exposure, and genetics. Neuropsychiatric disease and treatment. 2020;16:43.

38. Huang J, Li X, He W, Wan Y, Bao H, Xu Y, et al. Formation Environment and Development Models for the Lower Cambrian Source Rocks of the Southern North China Plate, China. ACS omega. 2020;5(14):8001-11.

39. Sheriff RS, Van Hooff M, Malhi G, Grace B, McFarlane A. Childhood trauma and childhood mental disorder in military and employed civilian men. The Journal of nervous and mental disease. 2020;208(1):13-20.

40. Thériault FL, Gardner W, Momoli F, Garber BG, Kingsbury M, Clayborne Z, et al. Mental health service use in depressed military personnel: a systematic review. Military medicine. 2020;185(7-8):e1255-e62.

41. Thériault FL, Hawes R, Garber B, Momoli F, Gardner W, Zamorski M, et al. Incidence of major depression diagnoses in the Canadian Armed Forces: longitudinal analysis of clinical and health administrative data. Social psychiatry and psychiatric epidemiology. 2020;55(5):581-8.

42. Kim Halford W. The future of couple relationship education: Suggestions on how it can make a difference. Family Relations. 2004;53(5):55966.

43. Busby DM, Ivey DC, Harris SM, Ates C. Self-directed, therapist-directed, and assessment-based interventions for premarital couples. Family Relations. 2007;56(3):279-90

44. Ooms T, Wilson P. The challenges of offering relationship and marriage education to low-income populations. Family Relations. 2004;53(5):440-7.

45. Živić B, Joković D, Vranić M, Stojanović Z. Post-traumatic stress disorder psychotic subtype or comorbid psychotic disorder and evaluation of military service ability. Vojnosanitetski pregled. 2020;77(3):335-9.

46. Al-Turkait FA, Ohaeri JU. Prevalence and correlates of posttraumatic stress disorder among Kuwaiti military men according to level of involvement in the first Gulf War. Depression and anxiety. 2008;25(11):932-41.

47. Kamm B, A. Depressive, aggressive and paranoid reactions. Psychoanalytic review. 1951;38(2):127-38.

48. Fisch M. The suicidal gesture: A study of 114 military patients hospitalized because of abortive suicide attempts. American Journal of Psychiatry. 1954;111(1):33-6.

49. OFFENKRANTZ W, CHURCH E, ELLIOTT R. Psychiatric management of suicide problems in military service. American Journal of Psychiatry. 1957;114(1):33-41.

50. Drehner D, Neuhauser KM, Neuhauser TS, Drehner D, Blackwood GV. Death among US Air Force basic trainees, 1956 to 1996. Military medicine. 1999;164(12):841-7.

51. Slu P. Mental disorders in men enlisted into military service and in soldiers (clinico-epidemiological study). Zhurnal nevrologii i psikhiatrii imeni SS Korsakova. 1999;99(8):16-20.

52. Peltzer K. Trauma and mental health problems of Sudanese refugees in Uganda. The Central African journal of medicine. 1999;45(5):110-4.

53. Rossow I, Romelsjo A, Leifman $\mathrm{H}$. Alcohol abuse and suicidal behaviour in young and middle aged men: differentiating between attempted and completed suicide. Addiction (Abingdon, England). 1999;94(8):1199-207.

Page 38/50 
54. Stahre MA, Brewer RD, Fonseca VP, Naimi TS. Binge drinking among US active-duty military personnel. American journal of preventive medicine. 2009;36(3):208-17.

55. Ames GM, Cunradi CB, Moore RS, Stern P. Military culture and drinking behavior among U.S. Navy careerists. Journal of studies on alcohol and drugs. 2007;68(3):336-44.

56. Bray RM, Hourani LL. Substance use trends among active duty military personnel: findings from the United States Department of Defense Health Related Behavior Surveys, 1980-2005. Addiction (Abingdon, England). 2007;102(7):1092-101.

57. Grønkjaer M, Flensborg-Madsen T, Osler M, Sørensen HJ, Becker U, Mortensen EL. Intelligence Test Scores Before and After Alcohol-Related Disorders-A Longitudinal Study of Danish Male Conscripts. Alcoholism, clinical and experimental research. 2019;43(10):2187-95.

58. Young CM, Pedersen ER, Pearson AD, Neighbors C. Drinking to cope moderates the efficacy of changing veteran drinking norms as a strategy for reducing drinking and alcohol-related problems among U.S. veterans. Psychology of addictive behaviors : journal of the Society of Psychologists in Addictive Behaviors. 2018;32(2):213-23.

59. Derefinko KJ, Klesges RC, Bursac Z, Little MA, Hryshko-Mullen A, Talcott GW. Alcohol issues prior to training in the United States Air Force. Addictive behaviors. 2016;58:142-8.

60. Wang L, Seelig A, Wadsworth SM, McMaster H, Alcaraz JE, Crum-Cianflone NF. Associations of military divorce with mental, behavioral, and physical health outcomes. BMC psychiatry. 2015;15:128.

61. Williams EC, Frasco MA, Jacobson IG, Maynard C, Littman AJ, Seelig AD, et al. Risk factors for relapse to problem drinking among current and former US military personnel: a prospective study of the Millennium Cohort. Drug and alcohol dependence. 2015;148:93-101.

62. Bray RM, Brown JM, Williams J. Trends in binge and heavy drinking, alcohol-related problems, and combat exposure in the U.S. military. Substance use \& misuse. 2013;48(10):799-810.

63. Farsi Z, JABARI MM, Saghiri Z. The relationship between depression with self injury in Army soldiers seen in a Military Medical Outpatient Clinic in Tehran. 2010.

64. Rohani S, Donyavi V, Shafighi F, Kazemi J, HOSSEINI S, HOSSEINI TS, et al. THE FREQUENCY OF SUICIDAL-THINKING IN NEZAJA PERSONNEL IN TEHRAN AT 2004-5 YEAR. 2006.

65. Tredgold RF. Depressive States in the Soldier: Their Symptoms, Causation, and Prognosis. British medical journal. 1941;2(4203):109-12.

66. Helzer JE, Robins LN, Davis DH. Depressive disorders in Vietnam returnees. The Journal of nervous and mental disease. 1976;163(3):177-85.

67. Levine ME. Depression in psychiatric admissions to a military medical center. Military medicine. 1982;147(9):752-5.

68. Deeken MG, Bridenbaugh RH. Depression and nightmares among Vietnam veterans in a military psychiatry outpatient clinic. Military medicine. 1987;152(11):590-1.

69. Ritchie EC, Radke AQ, Ross B. Depression and support systems in male army HIV+ patients. Military medicine. 1992;157(7):345-9.

70. Brown GR, Rundell JR, McManis SE, Kendall SN, Jenkins RA. Neuropsychiatric morbidity in early HIV disease: implications for military occupational function. Vaccine. 1993;11(5):560-9.

71. McCarroll JE, Orman DT, Lundy AC. Differences in self- and supervisor-referrals to a military mental health clinic. Military medicine. 1993;158(11):705-8.

72. Perconte ST, Wilson AT, Pontius EB, Dietrick AL, Spiro KJ. Psychological and war stress symptoms among deployed and non-deployed reservists following the Persian Gulf War. Military medicine. 1993;158(8):516-21.

73. Serfaty E, Andrade J, D'Aquila H, Masautis AE, Foglia L. [Severe depression and risk factors in Buenos Aires]. Acta psiquiatrica y psicologica de America latina. 1995;41(1):35-9.

74. Lish JD, Zimmerman M, Farber NJ, Lush DT, Kuzma MA, Plescia G. Suicide screening in a primary care setting at a Veterans Affairs medical center. Psychosomatics. 1996;37(5):413-24.

75. Long N, MacDonald C, Chamberlain K. Prevalence of posttraumatic stress disorder, depression and anxiety in a community sample of New Zealand Vietnam War veterans. The Australian and New Zealand journal of psychiatry. 1996;30(2):253-6.

76. Schwartz DA. Self-reported illness and health status among Gulf War veterans: A population-based study. Journal of the American Medical Association. 1997;277(3):238-45.

77. David D, Kutcher GS, Jackson El, Mellman TA. Psychotic symptoms in combat-related posttraumatic stress disorder. Journal of Clinical Psychiatry. 1999;60(1):29-32.

78. Hankin CS, Spiro lii A, Miller DR, Kazis L. Mental disorders and mental health treatment among U.S. Department of Veterans Affairs outpatients: The Veterans Health Study. American Journal of Psychiatry. 1999;156(12):1924-30.

79. Hourani LL, Yuan H. The mental health status of women in the Navy and Marine Corps: preliminary findings from the Perceptions of Wellness and Readiness Assessment. Military medicine. 1999;164(3):174-81.

80. Curran GM, Flynn HA, Kirchner J, Booth BM. Depression after alcohol treatment as a risk factor for relapse among male veterans. Journal of substance abuse treatment. 2000;19(3):259-65. 
81. Menon AS, Campbell D, Ruskin P, Hebel JR. Depression, hopelessness, and the desire for life-saving treatments among elderly medically ill veterans. American Journal of Geriatric Psychiatry. 2000;8(4):333-42.

82. Kozarić-Kovacić D, Hercigonja DK, Grubisić-llić M. Posttraumatic stress disorder and depression in soldiers with combat experiences. Croatian medical journal. 2001;42(2):165-70.

83. Sayar K, Köse O, Ebrinç S, Çetin M. Hopelessness, depression and alexithymia in young Turkish soldiers suffering from alopecia areata. Dermatology and Psychosomatics. 2001;2(1):12-5.

84. Hunter CL, Hunter CM, West ET, Kinder MH, Carroll DW. Recognition of depressive disorders by primary care providers in a military medical setting. Military medicine. 2002;167(4):308-11.

85. Karel MJ, Moye J. Assessing depression in medically ill elderly male veterans: Item-scale properties across and within racial groups. Journal of Mental Health and Aging. 2002;8(2):121-38.

86. Kilbourne AM, Justice AC, Rollman BL, McGinnis KA, Rabeneck L, Weissman S, et al. Clinical importance of HIV and depressive symptoms among veterans with HIV infection. Journal of general internal medicine. 2002;17(7):512-20.

87. Lehman CL, Cheung RC. Depression, anxiety, post-traumatic stress, and alcohol-related problems among veterans with chronic hepatitis C. American Journal of Gastroenterology. 2002;97(10):2640-6.

88. Muir AJ, Provenzale D. A descriptive evaluation of eligibility for therapy among veterans with chronic hepatitis $C$ virus infection. Journal of Clinical Gastroenterology. 2002;34(3):268-71.

89. Nguyen HA, Miller Al, Dieperink E, Willenbring ML, Tetrick LL, Durfee JM, et al. Spectrum of disease in U.S. veteran patients with hepatitis C. American Journal of Gastroenterology. 2002;97(7):1813-20.

90. Black DW, Carney CP, Forman-Hoffman VL, Letuchy E, Peloso P, Woolson RF, et al. Depression in veterans of the first Gulf War and comparable military controls. Annals of clinical psychiatry : official journal of the American Academy of Clinical Psychiatrists. 2004;16(2):53-61.

91. Gerson S, Mistry R, Bastani R, Blow F, Gould R, Llorente M, et al. Symptoms of depression and anxiety (MHI) following acute medical/surgical hospitalization and post-discharge psychiatric diagnoses (DSM) in 839 geriatric US veterans. International Journal of Geriatric Psychiatry. 2004;19(12):1155-67.

92. Rowan PJ, Tabasi S, Abdul-Latif M, Kunik ME, El-Serag HB. Psychosocial factors are the most common contraindications for antiviral therapy at initial evaluation in veterans with chronic hepatitis C. Journal of Clinical Gastroenterology. 2004;38(6):530-4.

93. Smith TC, Smith B, Corbeil TE, Riddle JR, Ryan MAK. Self-reported mental health among US military personnel prior and subsequent to the terrorist attacks of September 11, 2001. Journal of occupational and environmental medicine. 2004;46(8):775-82.

94. Vafaee B, Seidy A. Prevalence of depression among physically-disabled veterans in northwestern Iran. Iranian Journal of Medical Sciences. 2004;29(1):43-4.

95. Forman-Hoffman VL, Carney CP, Sampson TR, Peloso PM, Woolson RF, Black DW, et al. Mental health comorbidity patterns and impact on quality of life among veterans serving during the first Gulf War. Quality of life research : an international journal of quality of life aspects of treatment, care and rehabilitation. 2005;14(10):2303-14.

96. Goulet JL, Fultz SL, McGinnis KA, Justice AC. Relative prevalence of comorbidities and treatment contraindications in HIV-mono-infected and HIV/HCV-co-infected veterans. AIDS. 2005;19(SUPPL. 3):S99-S105.

97. Rowan PJ, Al-Jurdi R, Tavakoli-Tabasi S, Kunik ME, Satrom SL, El-Serag HB. Physical and psychosocial contributors to quality of life in veterans with hepatitis C not on antiviral therapy. Journal of Clinical Gastroenterology. 2005;39(8):731-6.

98. Williams RM, Turner AP, Hatzakis Jr M, Bowen JD, Rodriquez AA, Haselkorn JK. Prevalence and correlates of depression among veterans with multiple sclerosis. Neurology. 2005;64(1):75-80.

99. Xiong H, Zhang X, Zhang Y, Ma F, Li Y, Li L. An investigation of the prevalence of depressive symptoms in soldiers during military training. Preventive medicine. 2005;41(2):642-5.

100. Grieger TA, Cozza SJ, Ursano RJ, Hoge C, Martinez PE, Engel CC, et al. Posttraumatic stress disorder and depression in battle-injured soldiers. The American journal of psychiatry. 2006;163(10):1777-83; quiz 860.

101. Hoge CW, Auchterlonie JL, Milliken CS. Mental health problems, use of mental health services, and attrition from military service after returning from deployment to Iraq or Afghanistan. Journal of the American Medical Association. 2006;295(9):1023-32.

102. Kress AM, Peterson MR, Hartzell MC. Association between obesity and depressive symptoms among U.S. Military active duty service personnel, 2002. Journal of psychosomatic research. 2006;60(3):263-71.

103. Pflanz SE, Ogle AD. Job stress, depression, work performance, and perceptions of supervisors in military personnel. Military medicine. 2006;171(9):861-5.

104. Dove MB, Joseph HJ. Sociodemographic profile of women entering a military substance use disorder treatment center. Military medicine. 2007;172(3):283-7.

105. Kolkow TT, Spira JL, Morse JS, Grieger TA. Post-traumatic stress disorder and depression in health care providers returning from deployment to Iraq and Afghanistan. Military medicine. 2007;172(5):451-5.

Page $40 / 50$ 
106. Warner CM, Warner CH, Breitbach J, Rachal J, Matuszak T, Grieger TA. Depression in entry-level military personnel. Military medicine. 2007;172(8):795-9.

107. Hoge CW, Castro CA, Messer SC, McGurk D, Cotting DI, Koffman RL. Combat duty in Iraq and Afghanistan, mental health problems and barriers to care. US Army Medical Department journal. 2008:7-17.

108. Iversen AC, van Staden L, Hughes JH, Browne T, Hull L, Hall J, et al. The prevalence of common mental disorders and PTSD in the UK military: using data from a clinical interview-based study. BMC psychiatry. 2009;9:68.

109. Kline A, Callahan L, Butler M, St. Hill L, Losonczy MF, Smelson DA. The relationship between military service eras and psychosocial treatment needs among homeless veterans with a co-occurring substance abuse and mental health disorder. Journal of Dual Diagnosis. 2009;5(3-4):35774.

110. Rehn LM, Meririnne E, Höök-Nikanne J, Isometsä E, Henriksson M. Depressive symptoms and suicidal ideation during isotretinoin treatment: a 12-week follow-up study of male Finnish military conscripts. Journal of the European Academy of Dermatology and Venereology : JEADV. 2009;23(11):1294-7.

111. Rukskul I, Leelahanaj T, Hirunviwatgul N, Pholboonyaruk A. The prevalence of common mental disorders among outpatient Thai army personnel. Journal of the Medical Association of Thailand = Chotmaihet thangphaet. 2009;92 Suppl 1:S60-6.

112. Rukskul I. The prevalence of common mental disorders among inpatient Thai Army Personnel. Journal of the Medical Association of Thailand = Chotmaihet thangphaet. 2010;93 Suppl 6:S6-12.

113. Fikretoglu D, Liu A, Pedlar D, Brunet A. Patterns and predictors of treatment delay for mental disorders in a nationally representative, active Canadian military sample. Medical care. 2010;48(1):10-7.

114. Haskell SG, Gordon KS, Mattocks K, Duggal M, Erdos J, Justice A, et al. Gender differences in rates of depression, PTSD, pain, obesity, and military sexual trauma among Connecticut War Veterans of Iraq and Afghanistan. Journal of women's health (2002). 2010;19(2):267-71.

115. Luxton DD, Skopp NA, Maguen S. Gender differences in depression and PTSD symptoms following combat exposure. Depression and anxiety. 2010;27(11):1027-33.

116. Maguen S, Ren L, Bosch JO, Marmar CR, Seal KH. Gender differences in mental health diagnoses among Iraq and Afghanistan veterans enrolled in veterans affairs health care. American journal of public health. 2010;100(12):2450-6.

117. Stecker T, Fortney J, Owen R, McGovern MP, Williams S. Co-occurring medical, psychiatric, and alcohol-related disorders among veterans returning from Iraq and Afghanistan. Psychosomatics. 2010;51(6):503-7.

118. Burnett-Zeigler I, Ilgen M, Valenstein M, Zivin K, Gorman L, Blow A, et al. Prevalence and correlates of alcohol misuse among returning Afghanistan and Iraq Veterans. Addictive behaviors. 2011;36(8):801-6.

119. Erbes CR, Kaler ME, Schult T, Polusny MA, Arbisi PA. Mental health diagnosis and occupational functioning in National Guard/Reserve veterans returning from Iraq. Journal of rehabilitation research and development. 2011;48(10):1159-70.

120. Guerra VS, Calhoun PS. Examining the relation between posttraumatic stress disorder and suicidal ideation in an OEF/OIF veteran sample. Journal of anxiety disorders. 2011;25(1):12-8.

121. Jakupcak M, Hoerster KD, Varra A, Vannoy S, Felker B, Hunt S. Hopelessness and suicidal ideation in Iraq and Afghanistan War Veterans reporting subthreshold and threshold posttraumatic stress disorder. The Journal of nervous and mental disease. 2011;199(4):272-5.

122. Kehle SM, Reddy MK, Ferrier-Auerbach AG, Erbes CR, Arbisi PA, Polusny MA. Psychiatric diagnoses, comorbidity, and functioning in National Guard troops deployed to Iraq. Journal of psychiatric research. 2011;45(1):126-32.

123. Garber BG, Zamorski MA, Jetly R. Mental health of Canadian Forces members while on deployment to Afghanistan. Canadian journal of psychiatry Revue canadienne de psychiatrie. 2012;57(12):736-44.

124. Maguen S, Cohen B, Ren L, Bosch J, Kimerling R, Seal K. Gender differences in military sexual trauma and mental health diagnoses among Iraq and Afghanistan veterans with posttraumatic stress disorder. Women's health issues : official publication of the Jacobs Institute of Women's Health. 2012;22(1):e61-6.

125. Vasterling JJ, Brailey K, Proctor SP, Kane R, Heeren T, Franz M. Neuropsychological outcomes of mild traumatic brain injury, post-traumatic stress disorder and depression in Iraq-deployed US Army soldiers. The British journal of psychiatry : the journal of mental science. 2012;201(3):186-92.

126. Cohen SI, Suri P, Amick MM, Yan K. Clinical and demographic factors associated with employment status in US military veterans returning from Iraq and Afghanistan. Work (Reading, Mass). 2013;44(2):213-9.

127. Harbertson J, Grillo M, Zimulinda E, Murego C, Cronan T, May S, et al. Prevalence of PTSD and depression, and associated sexual risk factors, among male Rwanda Defense Forces military personnel. Tropical medicine \& international health : TM \& IH. 2013;18(8):925-33.

128. Marshall BD, Prescott MR, Liberzon I, Tamburrino MB, Calabrese JR, Galea S. Posttraumatic stress disorder, depression, and HIV risk behavior among Ohio Army National Guard Soldiers. Journal of traumatic stress. 2013;26(1):64-70.

129. Morrow CE, Bryan CJ, Stephenson JA, Bryan AO, Haskell J, Staal M. Posttraumatic stress, depression, and insomnia among U.S. air force pararescuemen. Military Psychology. 2013;25(6):568-76. 
130. Swinkels CM, Ulmer CS, Beckham JC, Buse N, Calhoun PS. The Association of Sleep Duration, Mental Health, and Health Risk Behaviors among U.S. Afghanistan/Iraq Era Veterans. Sleep. 2013;36(7):1019-25.

131. Chapman PL, Elnitsky C, Pitts B, Figley C, Thurman RM, Unwin B. Mental health, help seeking, and stigma and barriers to care among 3- and 12-month postdeployed and never deployed U.S. Army Combat Medics. Military medicine. 2014;179(8 Suppl):55-62.

132. Clarke-Walper K, Riviere LA, Wilk JE. Alcohol misuse, alcohol-related risky behaviors, and childhood adversity among soldiers who returned from Iraq or Afghanistan. Addictive behaviors. 2014;39(2):414-9.

133. Curry JF, Aubuchon-Endsley N, Brancu M, Runnals JJ, Fairbank JA. Lifetime major depression and comorbid disorders among current-era women veterans. Journal of affective disorders. 2014;152-154:434-40.

134. Denneson LM, Corson K, Helmer DA, Bair MJ, Dobscha SK. Mental health utilization of new-to-care Iraq and Afghanistan Veterans following suicidal ideation assessment. Psychiatry research. 2014;217(3):147-53.

135. Don Richardson J, Cyr KS, Nelson C, Elhai JD, Sareen J. Sleep disturbances and suicidal ideation in a sample of treatment-seeking Canadian Forces members and veterans. Psychiatry research. 2014;218(1-2):118-23.

136. Garber BG, Rusu C, Zamorski MA. Deployment-related mild traumatic brain injury, mental health problems, and post-concussive symptoms in Canadian armed forces personnel. BMC psychiatry. 2014;14(1).

137. Heltemes KJ, Clouser MC, MacGregor AJ, Norman SB, Galarneau MR. Co-occurring mental health and alcohol misuse: dual disorder symptoms in combat injured veterans. Addictive behaviors. 2014;39(2):392-8.

138. Lehavot K, Simpson TL. Trauma, posttraumatic stress disorder, and depression among sexual minority and heterosexual women veterans. Journal of counseling psychology. 2014;61(3):392-403.

139. Ramsawh HJ, Fullerton CS, Mash HB, Ng TH, Kessler RC, Stein MB, et al. Risk for suicidal behaviors associated with PTSD, depression, and their comorbidity in the U.S. Army. Journal of affective disorders. 2014;161:116-22.

140. Bin Zubair U, Mansoor S, Rana MH. Prevalence of depressive symptoms and associated socio-demographic factors among recruits during military training. Journal of the Royal Army Medical Corps. 2015;161(2):127-31.

141. Cleveland SD, Branscum AJ, Bovbjerg VE, Thorburn S. Mental Health Symptoms Among Student Service Members/Veterans and Civilian College Students. Journal of American college health : J of ACH. 2015;63(7):459-72.

142. Foote CE, Kinnon JM, Robbins C, Pessagno R, Portner MD. Long-term health and quality of life experiences of Vietnam veterans with combatrelated limb loss. Quality of life research : an international journal of quality of life aspects of treatment, care and rehabilitation. 2015;24(12):2853-61.

143. Hamilton AB, Williams L, Washington DL. Military and mental health correlates of unemployment in a national sample of women veterans. Medical care. 2015;53(4 Suppl 1):S32-8.

144. Hoerster KD, Jakupcak M, Hanson R, McFall M, Reiber G, Hall KS, et al. PTSD and depression symptoms are associated with binge eating among US Iraq and Afghanistan veterans. Eating behaviors. 2015;17:115-8.

145. Kim NY, Lee PK, Lim MH. Suicidal idea, ADHD, depression, anxiety, self-esteem and impulsiveness in Korean soldiers. African Journal of Psychiatry (South Africa). 2015;18(5).

146. Lundin A, Mortensen LH. Mortality from alcohol consumption and alcohol use disorder: Findings from the Vietnam Experience Study. Drug and alcohol dependence. 2015;151:135-43.

147. McGuire A, Dobson A, Mewton L, Varker T, Forbes D, Wade D. Mental health service use: comparing people who served in the military or received Veterans' Affairs benefits and the general population. Australian and New Zealand journal of public health. 2015;39(6):524-9.

148. Mysliwiec V, Capaldi VF, 2nd, Gill J, Baxter T, O'Reilly BM, Matsangas P, et al. Adherence to positive airway pressure therapy in U.S. military personnel with sleep apnea improves sleepiness, sleep quality, and depressive symptoms. Military medicine. 2015;180(4):475-82.

149. Nasioudis D, Palaiodimos L, Dagiasis M, Katsarou A, Ntouros E. Depression in military medicine cadets: a cross-sectional study. Military Medical Research. 2015;2:28.

150. Vanderploeg RD, Nazem S, Brenner LA, Belanger HG, Donnell AJ, Scott SG. Suicidal Ideation among Florida National Guard Members: Combat Deployment and Non-Deployment Risk and Protective Factors. Archives of suicide research : official journal of the International Academy for Suicide Research. 2015;19(4):453-71.

151. Fink DS, Calabrese JR, Liberzon I, Tamburrino MB, Chan P, Cohen GH, et al. Retrospective age-of-onset and projected lifetime prevalence of psychiatric disorders among U.S. Army National Guard soldiers. Journal of affective disorders. 2016;202:171-7.

152. Forbes D, O'Donnell M, Brand RM, Korn S, Creamer M, McFarlane AC, et al. The long-term mental health impact of peacekeeping: prevalence and predictors of psychiatric disorder. BJPsych open. 2016;2(1):32-7.

153. Güloğlu B. Psychiatric symptoms of Turkish combat-injured non-professional veterans. European journal of psychotraumatology. 2016;7:29157.

154. Hardos JE, Whitehead LW, Han I, Ott DK, Waller DK. Depression Prevalence and Exposure to Organophosphate Esters in Aircraft Maintenance Workers. Aerospace medicine and human performance. 2016;87(8):712-7.

Page $42 / 50$ 
155. Herberman Mash HB, Fullerton CS, Ng TH, Nock MK, Wynn GH, Ursano RJ. Alcohol Use and Reasons for Drinking as Risk Factors for Suicidal Behavior in the U.S. Army. Military medicine. 2016;181(8):811-20.

156. Monteith LL, Bahraini NH, Matarazzo BB, Gerber HR, Soberay KA, Forster JE. The influence of gender on suicidal ideation following military sexual trauma among Veterans in the Veterans Health Administration. Psychiatry research. 2016;244:257-65.

157. Phillips KM, Clark ME, Gironda RJ, McGarity S, Kerns RW, Elnitsky CA, et al. Pain and psychiatric comorbidities among two groups of Iraq and Afghanistan era Veterans. Journal of rehabilitation research and development. 2016;53(4):413-32.

158. Zamorski MA, Bennett RE, Rusu C, Weeks M, Boulos D, Garber BG. Prevalence of Past-Year Mental Disorders in the Canadian Armed Forces, 2002-2013. Canadian journal of psychiatry Revue canadienne de psychiatrie. 2016;61(1 Suppl):26s-35s.

159. Boakye EA, Buchanan P, Wang J, Stringer L, Geneus C, Scherrer JF. Self-Reported Lifetime Depression and Current Mental Distress Among Veterans Across Service Eras. Military medicine. 2017;182(3):e1691-e6.

160. Cohen GH, Fink DS, Sampson L, Tamburrino M, Liberzon I, Calabrese JR, et al. Coincident alcohol dependence and depression increases risk of suicidal ideation among Army National Guard soldiers. Annals of epidemiology. 2017;27(3):157-63.e1.

161. Gradus JL, King MW, Galatzer-Levy I, Street AE. Gender Differences in Machine Learning Models of Trauma and Suicidal Ideation in Veterans of the Iraq and Afghanistan Wars. Journal of traumatic stress. 2017;30(4):362-71.

162. Packnett ER, Elmasry H, Toolin CF, Cowan DN, Boivin MR. Epidemiology of Major Depressive Disorder Disability in the US Military: FY 20072012. The Journal of nervous and mental disease. 2017;205(9):672-8.

163. Weeks M, Zamorski MA, Rusu C, Colman I. Mental illness-related stigma in canadian military and civilian populations: A comparison using population health survey data. Psychiatric Services. 2017;68(7):710-6.

164. Bartlett BA, Smith LJ, Tran JK, Vujanovic AA. Understanding mental health among military veterans in the fire service. Psychiatry research. 2018;267:394-9.

165. Blakey SM, Wagner HR, Naylor J, Brancu M, Lane I, Sallee M, et al. Chronic Pain, TBI, and PTSD in Military Veterans: A Link to Suicidal Ideation and Violent Impulses? The journal of pain : official journal of the American Pain Society. 2018;19(7):797-806.

166. Boulos D, Fikretoglu D. Influence of military component and deployment-related experiences on mental disorders among Canadian military personnel who deployed to Afghanistan: a cross-sectional survey. BMJ open. 2018;8(3):e018735.

167. Dillon KH, Cunningham KC, Neal JM, Wilson SM, Dedert EA, Elbogen EB, et al. Examination of the indirect effects of combat exposure on suicidal behavior in veterans. Journal of affective disorders. 2018;235:407-13.

168. Don Richardson J, King L, St. Cyr K, Shnaider P, Roth ML, Ketcheson F, et al. Depression and the relationship between sleep disturbances, nightmares, and suicidal ideation in treatment-seeking Canadian Armed Forces members and veterans. BMC psychiatry. 2018;18(1).

169. Elbogen EB, Wagner HR, Brancu M, Kimbrel NA, Naylor JC, Swinkels CM, et al. Psychosocial Risk Factors and Other Than Honorable Military Discharge: Providing Healthcare to Previously Ineligible Veterans. Military medicine. 2018;183(9-10):e532-e8.

170. Hourani LL, Williams J, Lattimore PK, Morgan JK, Hopkinson SG, Jenkins L, et al. Workplace victimization risk and protective factors for suicidal behavior among active duty military personnel. Journal of affective disorders. 2018;236:45-51.

171. Kizilhan JI, Noll-Hussong M. Post-traumatic stress disorder among former Islamic State child soldiers in northern Iraq. The British journal of psychiatry : the journal of mental science. 2018;213(1):425-9.

172. McDonald SD, Mickens MN, Goldberg-Looney LD, Mutchler BJ, Ellwood MS, Castillo TA. Mental disorder prevalence among U.S. Department of Veterans Affairs outpatients with spinal cord injuries. The journal of spinal cord medicine. 2018;41(6):691-702.

173. Stefanovics EA, Potenza MN, Pietrzak RH. The physical and mental health burden of obesity in U.S. veterans: Results from the National Health and Resilience in Veterans Study. Journal of psychiatric research. 2018;103:112-9.

174. Vun E, Turner S, Sareen J, Mota N, Afifi TO, El-Gabalawy R. Prevalence of comorbid chronic pain and mental health conditions in Canadian Armed Forces active personnel: analysis of a cross-sectional survey. CMAJ open. 2018;6(4):E528-e36.

175. Waitzkin H, Cruz M, Shuey B, Smithers D, Muncy L, Noble M. Military Personnel Who Seek Health and Mental Health Services Outside the Military. Military medicine. 2018;183(5-6):e232-e40.

176. Byrne SP, Harpaz-Rotem I, Tsai J, Southwick SM, Pietrzak RH. Latent typologies of DSM-5 PTSD symptoms in U.S. military veterans. Psychiatry research. 2019;273:266-73.

177. Carney B, White J, Xu X, Sunil T, Daniels C, Byrne M, et al. Relationship between depression and risk behaviors in a US Military population with HIV infection. AIDS care. 2019;31(9):1152-6.

178. Jones N, Greenberg N, Phillips A, Simms A, Wessely S. Mental Health, Help-Seeking Behaviour and Social Support in the UK Armed Forces by Gender. Psychiatry. 2019;82(3):256-71.

179. Lucas CL, Cederbaum JA, Kintzle S, Castro CA. An Examination of Stalking Experiences During Military Service Among Female and Male Veterans and Associations With PTSD and Depression. Journal of interpersonal violence. 2019:886260519889944.

180. Nichter B, Norman S, Haller M, Pietrzak RH. Psychological burden of PTSD, depression, and their comorbidity in the U.S. veteran population: Suicidality, functioning, and service utilization. Journal of affective disorders. 2019;256:633-40.

Page $43 / 50$ 
181. Start AR, Allard Y, Adler A, Toblin R. Predicting Suicide Ideation in the Military: The Independent Role of Aggression. Suicide \& life-threatening behavior. 2019;49(2):444-54.

182. Blosnich JR, Montgomery AE, Dichter ME, Gordon AJ, Kavalieratos D, Taylor L, et al. Social Determinants and Military Veterans' Suicide Ideation and Attempt: a Cross-sectional Analysis of Electronic Health Record Data. Journal of general internal medicine. 2020;35(6):1759-67.

183. Forys-Donahue KL, Brooks RD, Beymer MR, Pecko J. The association between nutrition and behavioural health in a US Army population. Public health nutrition. 2020:1-8.

184. Gjerstad CL, Bøe HJ, Falkum E, Martinsen EW, Nordstrand AE, Tønnesen A, et al. Prevalence and Correlates of Mental Health Problems in Norwegian Peacekeepers 18-38 Years Postdeployment. Journal of traumatic stress. 2020.

185. Groll DL, Ricciardelli R, Carleton RN, Anderson G, Cramm H. A Cross-Sectional Study of the Relationship between Previous Military Experience and Mental Health Disorders in Currently Serving Public Safety Personnel in Canada. Canadian Journal of Psychiatry. 2020;65(5):330-7.

186. Gross GM, Bastian LA, Smith NB, Harpaz-Rotem I, Hoff R. Sex Differences in Associations Between Depression and Posttraumatic Stress Disorder Symptoms and Tobacco Use Among Veterans of Recent Conflicts. Journal of women's health (2002). 2020;29(5):677-85.

187. Shim EJ, Hwang H, Lee KM, Lee JY, Lee SD, Baik MJ, et al. Somatic symptoms and sleep quality as indicators of depression and suicide risk among Korean military conscripts. Psychiatry research. 2020;287:112912.

188. Smigelsky MA, Jardin C, Nieuwsma JA, Brancu M, Meador KG, Molloy KG, et al. Religion, spirituality, and suicide risk in Iraq and Afghanistan era veterans. Depression and anxiety. 2020;37(8):728-37.

189. Smith LM, Stanley IH, Joiner TE. Mental Health Outcomes of Premature Discharge from United States Air Force Basic Military Training. Military medicine. 2020.

190. Stefanovics EA, Potenza MN, Pietrzak RH. PTSD and obesity in U.S. military veterans: Prevalence, health burden, and suicidality. Psychiatry research. 2020;291:113242.

191. Taillieu TL, Afifi TO, Zamorski MA, Turner S, Cheung K, Stein MB, et al. Clinical Epidemiology of Alcohol Use Disorders in Military Personnel versus the General Population in Canada. Canadian Journal of Psychiatry. 2020;65(4):253-63.

192. Wang J, Ursano RJ, Gifford RK, Dinh H, Farooq S, Broshek CE, et al. Mental Health and Suicidality in Separating U.S. Reserve and National Guard Personnel. Psychiatry. 2020;83(2):166-75.

193. Ursano RJ, Herberman Mash HB, Kessler RC, Naifeh JA, Fullerton CS, Aliaga PA, et al. Factors Associated With Suicide Ideation in US Army Soldiers During Deployment in Afghanistan. JAMA network open. 2020;3(1):e1919935.

194. Yeom CW, Oh GH, Jung S, Moon JY, Son KL, Kim WH, et al. Prevalence and comorbidities of adult adhd in male military conscripts in korea: Results of an epidemiological survey of mental health in korean military service. Psychiatry research. 2020;293:113401.

195. Bohnker B, McEwen G, Blanco J, Feeks E. Psychiatric diagnoses aboard an aircraft carrier. Aviation, space, and environmental medicine. 1992;63(11):1015-8.

196. Benda BB. Discriminators of Suicide Thoughts and Attempts among Homeless Veterans Who Abuse Substances. Suicide and Life-Threatening Behavior. 2003;33(4):430-42.

197. Ritchie EC, Keppler WC, Rothberg JM. Suicidal admissions in the United States military. Military medicine. 2003;168(3):177-81.

198. Benda BB. Gender differences in predictors of suicidal thoughts and attempts among homeless veterans that abuse substances. Suicide \& lifethreatening behavior. 2005;35(1):106-16.

199. Belik SL, Stein MB, Asmundson GJG, Sareen J. Are canadian soldiers more likely to have suicidal ideation and suicide attempts than canadian civilians? American journal of epidemiology. 2010;172(11):1250-8.

200. Mansfield AJ, Bender RH, Hourani LL, Larson GE. Suicidal or self-harming ideation in military personnel transitioning to civilian life. Suicide \& life-threatening behavior. 2011;41(4):392-405.

201. Maguen S, Metzler TJ, Bosch J, Marmar CR, Knight SJ, Neylan TC. Killing in combat may be independently associated with suicidal ideation. Depression and anxiety. 2012;29(11):918-23.

202. Bryan CJ, Clemans TA. Repetitive traumatic brain injury, psychological symptoms, and suicide risk in a clinical sample of deployed military personnel. JAMA psychiatry. 2013;70(7):686-91.

203. Bryan CJ, Clemans TA, Hernandez AM, Rudd MD. Loss of consciousness, depression, posttraumatic stress disorder, and suicide risk among deployed military personnel with mild traumatic brain injury. The Journal of head trauma rehabilitation. 2013;28(1):13-20.

204. Bryan CJ, Hernandez AM. The functions of social support as protective factors for suicidal ideation in a sample of air force personnel. Suicide \& life-threatening behavior. 2013;43(5):562-73.

205. Bryan CJ, Morrow CE, Etienne N, Ray-Sannerud B. Guilt, shame, and suicidal ideation in a military outpatient clinical sample. Depression and anxiety. 2013;30(1):55-60.

206. Blosnich JR, Gordon AJ, Bossarte RM. Suicidal ideation and mental distress among adults with military service history: results from 5 U.S. states, 2010. American journal of public health. 2014;104 Suppl 4(Suppl 4):S595-602. 
207. Bryan CJ, Bryan AO, Ray-Sannerud BN, Etienne N, Morrow CE. Suicide attempts before joining the military increase risk for suicide attempts and severity of suicidal ideation among military personnel and veterans. Comprehensive psychiatry. 2014;55(3):534-41.

208. Mash HB, Fullerton CS, Ramsawh HJ, Ng TH, Wang L, Kessler RC, et al. Risk for suicidal behaviors associated with alcohol and energy drink use in the US Army. Social psychiatry and psychiatric epidemiology. 2014;49(9):1379-87.

209. Bryan CJ, Ray-Sannerud B, Heron EA. Psychological flexibility as a dimension of resilience for posttraumatic stress, depression, and risk for suicidal ideation among Air Force personnel. Journal of Contextual Behavioral Science. 2015;4(4):263-8.

210. Ursano RJ, Heeringa SG, Stein MB, Jain S, Raman R, Sun X, et al. Prevalence and correlates of suicidal behavior among new soldiers in the U.S. Army: Results from the Army Study to Assess Risk and Resilience in Servicemembers (Army STARRS). Depression and anxiety. 2015;32(1):312.

211. Kachadourian LK, Gandelman E, Ralevski E, Petrakis IL. Suicidal ideation in military veterans with alcohol dependence and PTSD: The role of hostility. The American journal on addictions. 2018;27(2):124-30.

212. Kerr K, Romaniuk M, McLeay S, Khoo A, Dent MT, Boshen M. Increased risk of attempted suicide in Australian veterans is associated with total and permanent incapacitation, unemployment and posttraumatic stress disorder severity. The Australian and New Zealand journal of psychiatry. 2018;52(6):552-60.

213. Cramer RJ, Franks M, Cunningham CA, Bryan CJ. Preferences in Information Processing: Understanding Suicidal Thoughts and Behaviors among Active Duty Military Service Members. Archives of suicide research : official journal of the International Academy for Suicide Research. 2020:1-18.

214. Anestis MD, Bandel SL, Butterworth SE, Bond AE, Daruwala SE, Bryan CJ. Suicide risk and firearm ownership and storage behavior in a large military sample. Psychiatry research. 2020;291:113277.

215. Monteith LL, Holliday R, Miller C, Schneider AL, Hoffmire CA, Bahraini NH, et al. Suicidal ideation, suicide attempt, and non-suicidal self-injury among female veterans: Prevalence, timing, and onset. Journal of affective disorders. 2020;273:350-7.

\section{Figures}




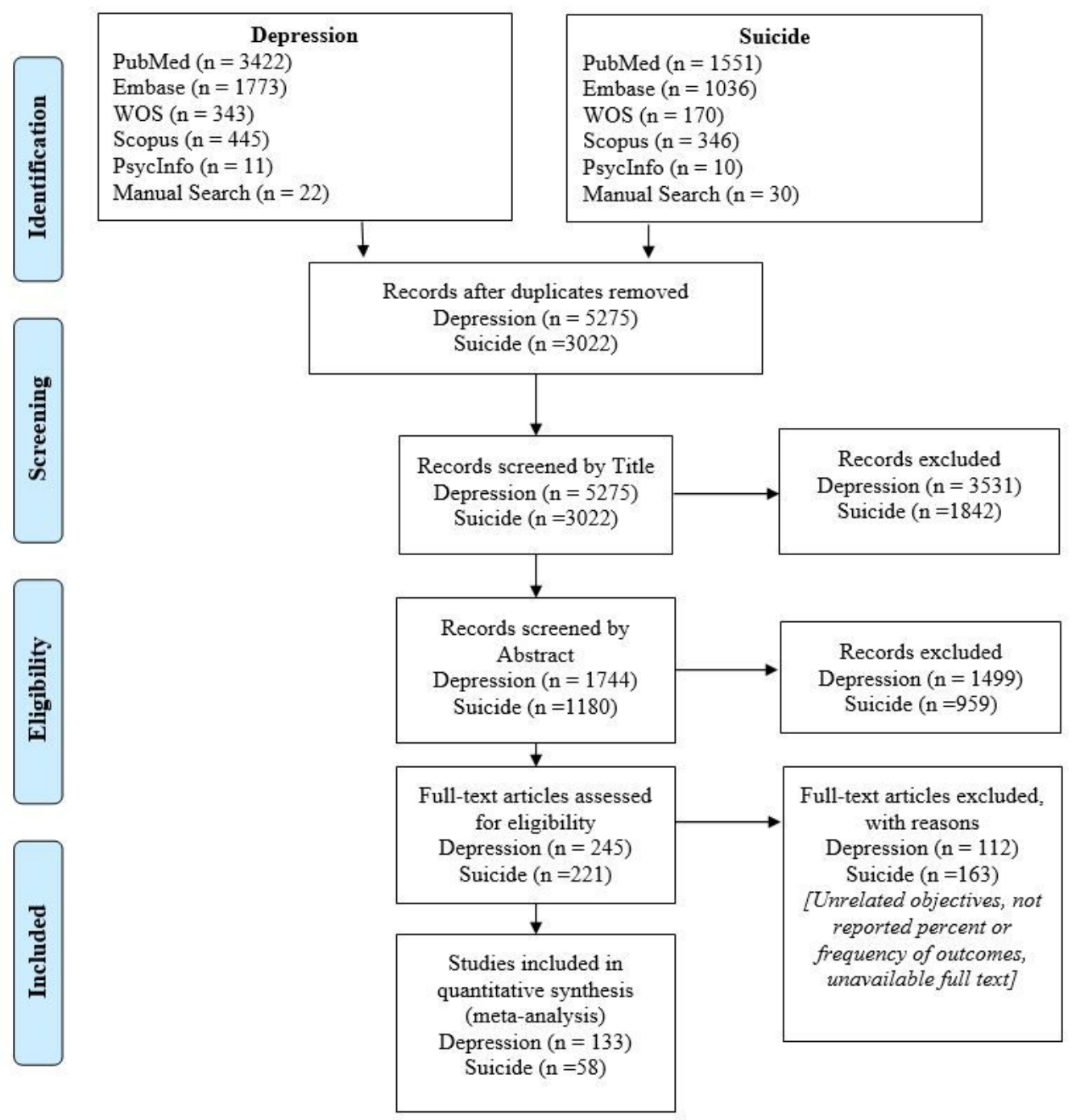

\section{Figure 1}

The Search Strategy Outputs and Screening Process based on Title, Abstract, and Full Text. 


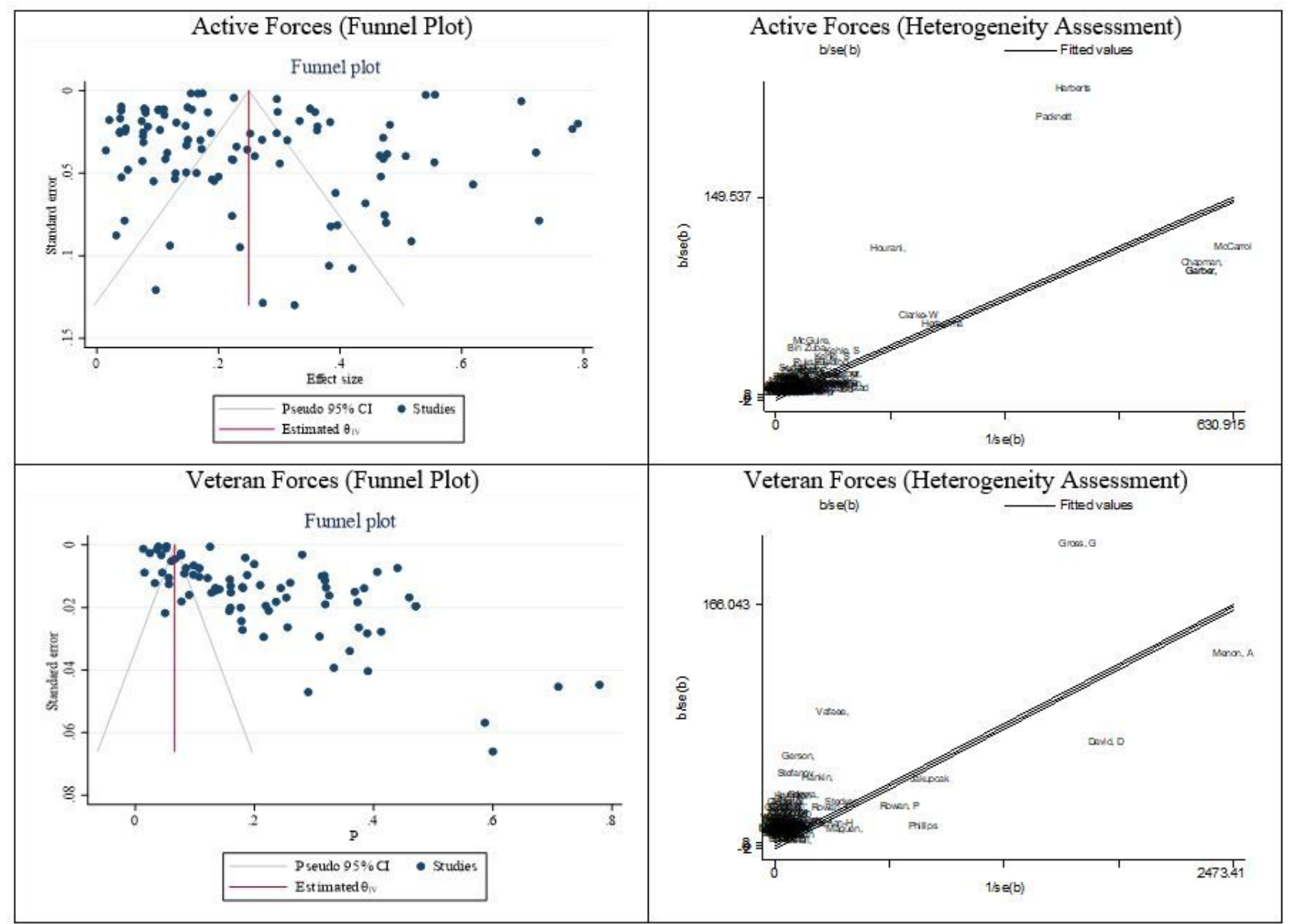

Figure 2

Results of Publication bias and Heterogeneity in pooled prevalence of depression in active duty and veteran military 


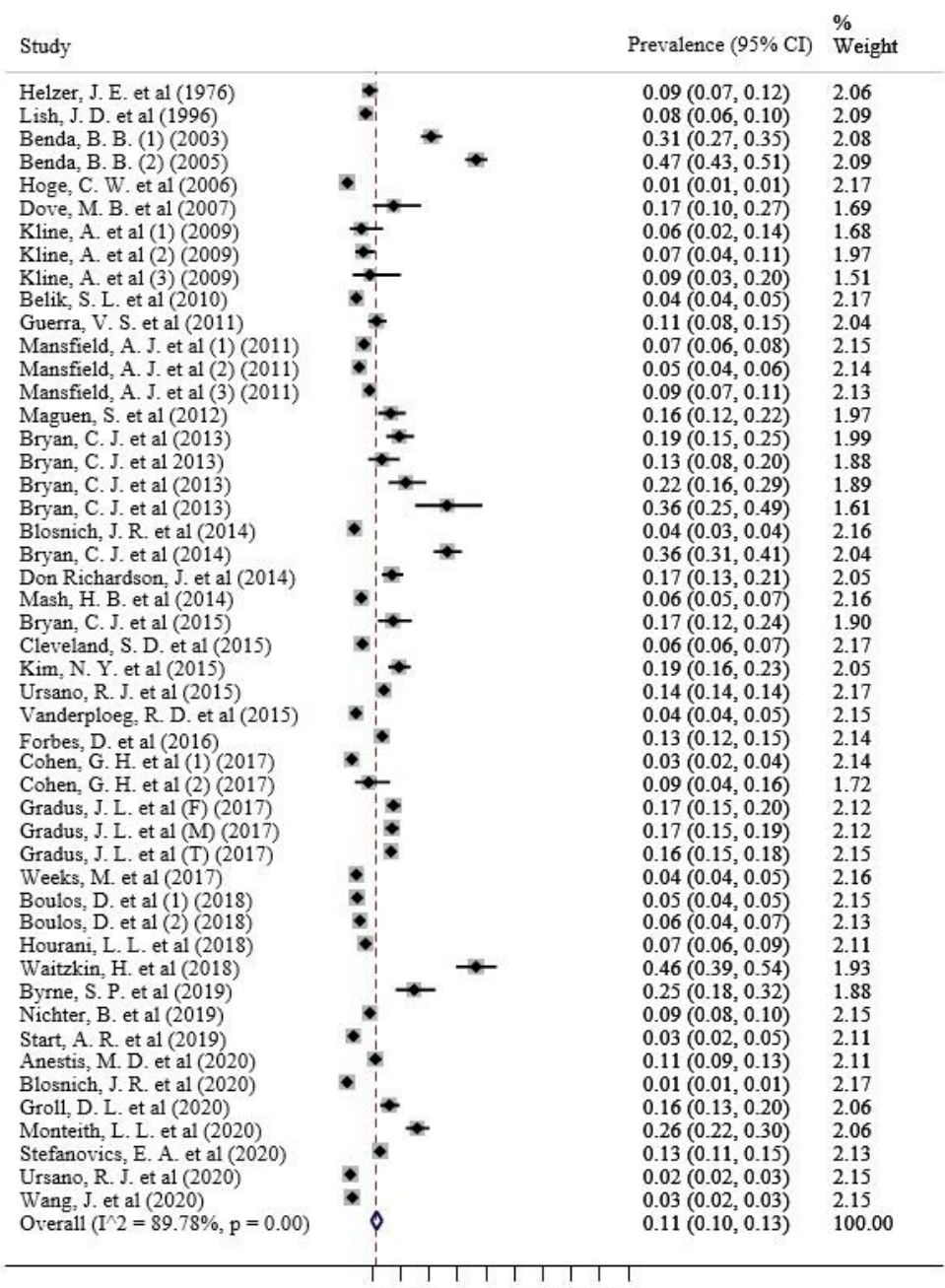

$\begin{array}{lllllllllll}.1 & 2 & 2 & 3 & .4 & .5 & .6 & .7 & .8 & .9 & 1\end{array}$

\section{Figure 3}

The pooled prevalence of Suicide thought in all military 


\begin{tabular}{|c|c|c|c|c|}
\hline Study & & & Prevalence $(95 \% \mathrm{CI})$ & $\begin{array}{l}\% \\
\text { Weight }\end{array}$ \\
\hline Bohnker, B. et al (1992) & i & $\rightarrow$ & $0.68(0.60,0.75)$ & 2.19 \\
\hline Brown, G. R. et al (1993) & -1 & & $0.05(0.04,0.08)$ & 2.43 \\
\hline Benda, B. B. (2003) & $i$ & $\leftarrow$ & $0.40(0.36,0.44)$ & 2.46 \\
\hline Ritchie, E. C. et al (2003) & $i$ & $\longrightarrow$ & $0.54(0.44,0.64)$ & 2.05 \\
\hline Benda, B. B. $(2005)$ & $i$ & $\leftarrow$ & $0.32(0.28,0.35)$ & 2.47 \\
\hline Kline, A. et al (2009) & 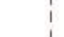 & $\leftarrow$ & $0.27(0.21,0.33)$ & 2.32 \\
\hline Kline, A et al (2009) & $i$ & $\rightarrow$ & $0.28(0.19,0.39)$ & 1.96 \\
\hline Kline, A. et al (2009) & $\perp$ & & $0.16(0.08,0.29)$ & 1.76 \\
\hline Belik, S. L. et al (2010) & 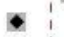 & & $0.01(0.01,0.01)$ & 2.57 \\
\hline Guerra, V. S. et al (2011) & - & & $0.09(0.06,0.12)$ & 2.41 \\
\hline Maguen, S. et al (2012) & -1 & & $0.05(0.03,0.08)$ & 2.33 \\
\hline Bryan, C. J. et al (2013) & -1 & & $0.02(0.00,0.05)$ & 2.21 \\
\hline Swinkels, C. M. et al (2013) & $\bullet$ & & $0.08(0.07,0.09)$ & 2.53 \\
\hline Bryan, C. J. et al (2014) & 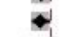 & & $0.08(0.05,0.11)$ & 2.41 \\
\hline Ramsawh, H. J. et al (2014) & $\bullet$ & & $0.06(0.06,0.07)$ & 2.56 \\
\hline Bryan, C. J. et al (2015) & -1 & & $0.01(0.00,0.04)$ & 2.23 \\
\hline Cleveland, S. D. et al (2015) & -1 & & $0.01(0.01,0.01)$ & 2.57 \\
\hline McGuire, A. et al (2015) & 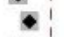 & & $0.05(0.04,0.05)$ & 2.56 \\
\hline McGuire, A et al (2015) & -1 & & $0.03(0.02,0.03)$ & 2.55 \\
\hline Ursano, R. J. et al (2015) & -1 & & $0.01(0.01,0.02)$ & 2.57 \\
\hline Forbes, D. et al (2016) & -1 & & $0.01(0.01,0.02)$ & 2.54 \\
\hline Herberman Mash, H. B. et al (2016) & $\bullet$ & & $0.08(0.07,0.10)$ & 2.52 \\
\hline Herberman Mash, H. B. et al (2016) & 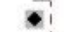 & & $0.06(0.05,0.07)$ & 2.55 \\
\hline Monteith, L. L. et al (F) (2016) & 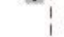 & $\rightarrow$ & $0.30(0.17,0.45)$ & 1.63 \\
\hline Monteith, L. L. et al (M) (2016) & 1 & $\rightarrow$ & $0.26(0.22,0.32)$ & 2.37 \\
\hline Monteith, L. L. et al (T) (2016) & i & $\rightarrow$ & $0.26(0.21,0.31)$ & 2.40 \\
\hline Bartlett, B. A. et al (2018) & -1 & & $0.07(0.06,0.09)$ & 2.50 \\
\hline Dillon, K. H. et al (2018) & 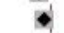 & & $0.09(0.08,0.10)$ & 2.55 \\
\hline Elbogen, E. B. et al (2018) & -1 & & $0.07(0.06,0.09)$ & 2.52 \\
\hline Hourani, L. L. et al (2018) & $\bullet$ & & $0.02(0.01,0.03)$ & 2.50 \\
\hline Kachadourian, L. K. et al (2018) & & $-\infty$ & $0.20(0.13,0.30)$ & 2.01 \\
\hline Kerr, K. et al (2018) & $i$ & 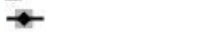 & $0.24(0.18,0.30)$ & 2.31 \\
\hline Byrne, S. P. et al (2019) & 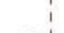 & $\rightarrow-$ & $0.25(0.19,0.33)$ & 2.21 \\
\hline Nichter, B. et al (2019) & $\bullet$ & & $0.05(0.04,0.06)$ & 2.55 \\
\hline Blosnich, J. R. et al (2020) & -1 & & $0.00(0.00,0.00)$ & 2.57 \\
\hline Cramer, R. J. et al (2020) & & $\rightarrow$ & $0.48(0.41,0.55)$ & 2.28 \\
\hline Groll, D. L. et al (2020) & -1 & & $0.04(0.02,0.06)$ & 2.44 \\
\hline Monteith, L. L. et al (2020) & i & $\leftarrow$ & $0.36(0.31,0.41)$ & 2.43 \\
\hline Shim, E. J. et al $(2020)$ & -1 & & $0.04(0.04,0.06)$ & 2.54 \\
\hline Smigelsky, M. A. et al (2020) & -1 & & $0.04(0.03,0.06)$ & 2.51 \\
\hline Stefanovics, E. A. et al $(2020)$ & $\bullet$ & & $0.09(0.08,0.11)$ & 2.52 \\
\hline Yeom, C. W. et al $(2020)$ & -1 & & $0.05(0.03,0.07)$ & 2.44 \\
\hline Overall $\left(\mathrm{I}^{\wedge} 2=79.55 \%, \mathrm{p}=0.05\right)$ & 0 & & $0.11(0.09,0.13)$ & 100.00 \\
\hline
\end{tabular}

\section{Figure 4}

The pooled prevalence of Suicide attempted in all military 


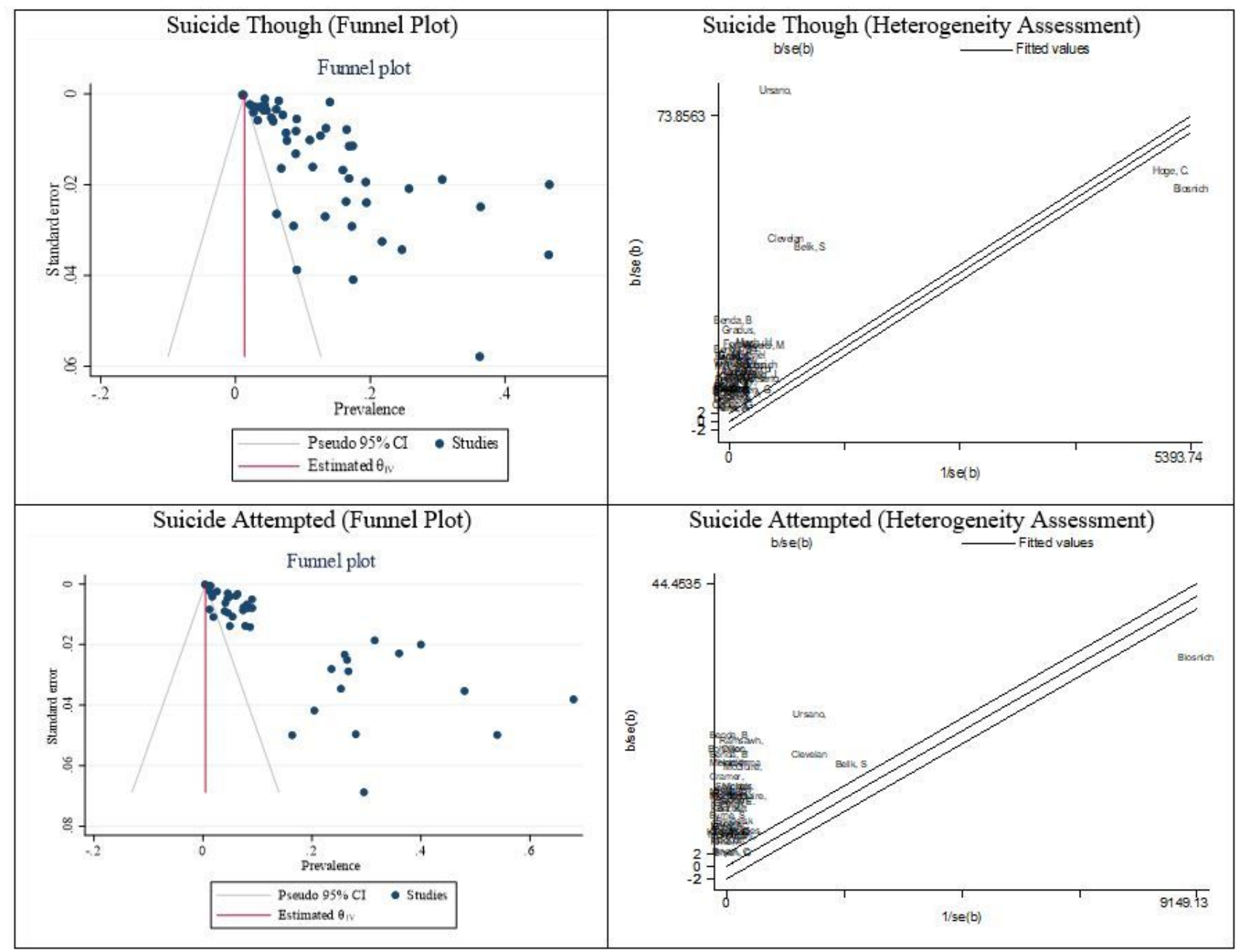

Figure 5

Results of Publication bias and Heterogeneity in pooled prevalence of Suicide though and attempted in all military 